University of Tennessee Health Science Center UTHSC Digital Commons

\title{
$5-2009$
}

\section{Biomechanical Comparison of Lumbar Disc Replacements}

Peter Wong

University of Tennessee Health Science Center

Follow this and additional works at: https://dc.uthsc.edu/dissertations

Part of the Equipment and Supplies Commons, Surgical Procedures, Operative Commons, and the Therapeutics Commons

\section{Recommended Citation}

Wong, Peter , "Biomechanical Comparison of Lumbar Disc Replacements" (2009). Theses and Dissertations (ETD). Paper 302. http://dx.doi.org/10.21007/etd.cghs.2009.0356.

This Thesis is brought to you for free and open access by the College of Graduate Health Sciences at UTHSC Digital Commons. It has been accepted for inclusion in Theses and Dissertations (ETD) by an authorized administrator of UTHSC Digital Commons. For more information, please contact jwelch30@uthsc.edu. 


\title{
Biomechanical Comparison of Lumbar Disc Replacements
}

\begin{abstract}
Surgical options for lumbar spinal disorders are rapidly expanding. One seemingly promising area is the lumbar disc replacement. Design specifications vary among the manufacturers based upon differing perceptions of ideal motion. Comparison studies are important in quantifying different features for future improvements but are currently lacking. The objective of this study was to compare the biomechanical properties of three currently available treatments: disc replacement with ProDisc-L, disc replacement with Maverick, and a pedicle screw fixation. It was hypothesized that structural designs differences of ProDisc$L$ and Maverick could be distinguished in both motion and loading patterns. It was also hypothesized that either disc replacements offered preservation of harvested motion at all motion levels whereas the pedicle screw fixation would not. Using a previously established non-destructive testing protocol, measurements of motion and flexibility were obtained. Seven human cadaver lumbar spines (L1-S1) were tested in flexion, extension, left and right lateral bending, and left and right axial rotation. Testing conditions, in order, were the harvested state, ProDisc-L at L5-S1, Maverick at L5-S1, and a pedicle screw fixation at L5-S1. ProDisc-L was less flexible than Maverick in flexion but otherwise similar. ProDisc-L and Maverick did not offer any differences in percent rotation contribution compared to the harvested state. Pedicle screw fixation both decreased the operative level and increased adjacent level rotation in flexionextension and axial rotation. ProDisc- $L$ and Maverick represent a similar class of disc replacements. The two devices are expected to offer similar outcomes in patients while fixation cannot.
\end{abstract}

\section{Document Type}

Thesis

Degree Name

Master of Science (MS)

Program

Biomedical Engineering and Imaging

Research Advisor

Denis DiAngelo, Ph.D.

Keywords

biomechanics, spine, disc replacement

\section{Subject Categories}

Analytical, Diagnostic and Therapeutic Techniques and Equipment | Equipment and Supplies | Medicine and Health Sciences | Surgical Procedures, Operative | Therapeutics 


\title{
BIOMECHANICAL COMPARISON OF LUMBAR DISC REPLACEMENTS
}

\author{
A Thesis \\ Presented for \\ The Graduate Studies Council \\ The University of Tennessee \\ Health Science Center
}

\author{
In Partial Fulfillment \\ Of the Requirements for the Degree \\ Master of Science \\ In the Joint Graduate Program in Biomedical Engineering and Imaging \\ From The University of Tennessee \\ and \\ The University of Memphis
}

By
Peter Wong
May 2009 
Copyright (C) 2009 by Peter Wong All rights reserved 


\section{ACKNOWLEDGMENTS}

I would like to thank my advisor, Dr. Denis J. DiAngelo, for the opportunity to work and learn under his guidance. I thank my committee members Dr. Gladius Lewis and Dr. Brian Kelly for their valuable input and support.

I owe a great deal to my fellow students for their advice and assistance, especially to Braham Dhillon, Brian Morrow, Jason Roberson, and Dan Wido. Finally, I thank Dr. Anthony Sin for this surgical support and the Medical Education Research Institute for allowing us to use their equipment. 


\begin{abstract}
Surgical options for lumbar spinal disorders are rapidly expanding. One seemingly promising area is the lumbar disc replacement. Design specifications vary among the manufacturers based upon differing perceptions of ideal motion. Comparison studies are important in quantifying different features for future improvements but are currently lacking. The objective of this study was to compare the biomechanical properties of three currently available treatments: disc replacement with ProDisc-L, disc replacement with Maverick, and a pedicle screw fixation. It was hypothesized that structural designs differences of ProDisc-L and Maverick could be distinguished in both motion and loading patterns. It was also hypothesized that either disc replacements offered preservation of harvested motion at all motion levels whereas the pedicle screw fixation would not. Using a previously established non-destructive testing protocol, measurements of motion and flexibility were obtained. Seven human cadaver lumbar spines (L1-S1) were tested in flexion, extension, left and right lateral bending, and left and right axial rotation. Testing conditions, in order, were the harvested state, ProDisc-L at L5-S1, Maverick at L5-S1, and a pedicle screw fixation at L5-S1. ProDisc-L was less flexible than Maverick in flexion but otherwise similar. ProDisc-L and Maverick did not offer any differences in percent rotation contribution compared to the harvested state. Pedicle screw fixation both decreased the operative level and increased adjacent level rotation in flexion-extension and axial rotation. ProDisc-L and Maverick represent a similar class of disc replacements. The two devices are expected to offer similar outcomes in patients while fixation cannot.
\end{abstract}




\section{TABLE OF CONTENTS}

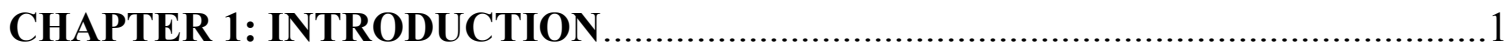

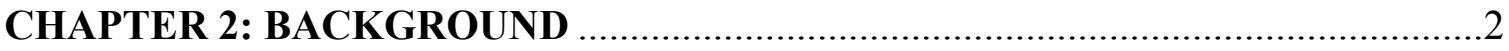

Anatomy of the Spine ..................................................................................

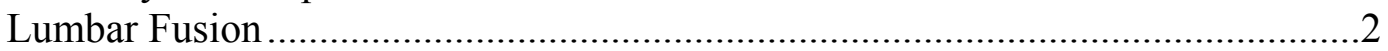

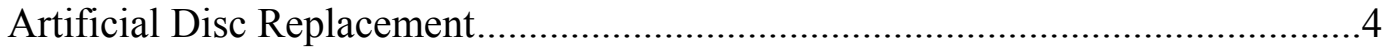

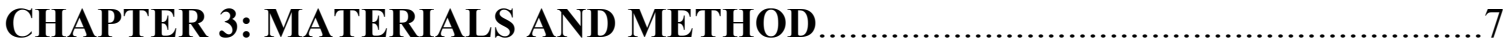

Specimen Preparation .................................................................................... 7

Summary of Instrumentation Notes ........................................................ 7

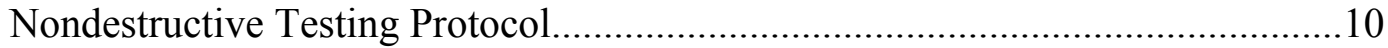

Data Management and Analysis ................................................................... 10

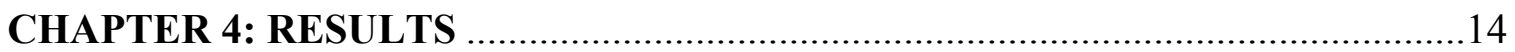

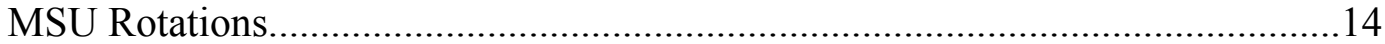

Global Stiffness/Normalized Flexibility ........................................................ 14

MSU Percent Change in Contribution .............................................................14

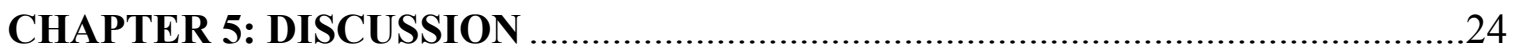

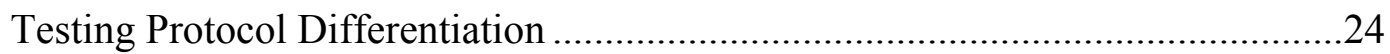

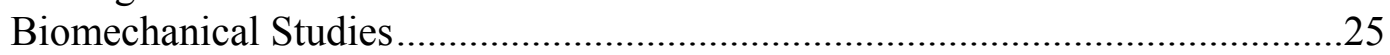

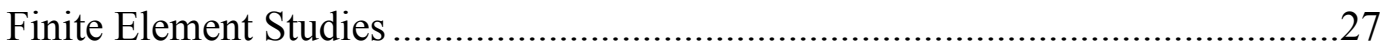

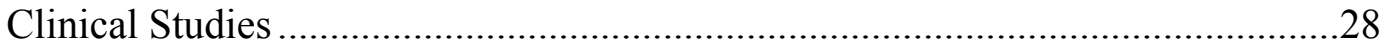

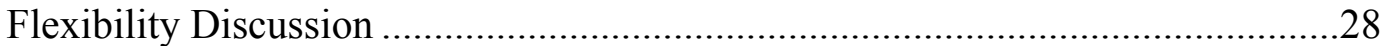

MSU Percent Change in Contribution .............................................................29

Adjacent Level Effects.........................................................................29

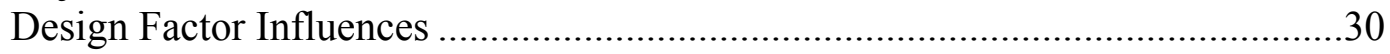

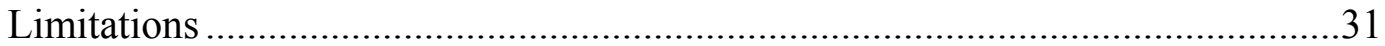

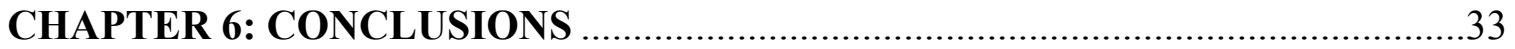

CHAPTER 7: RECOMMENDATIONS FOR FUTURE STUDY ............................34

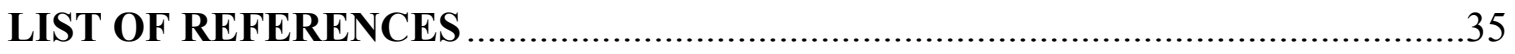

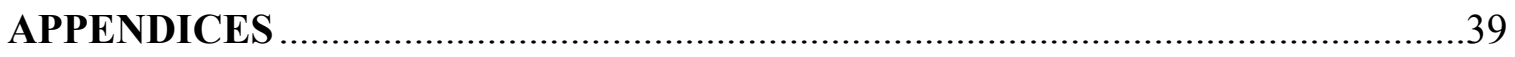

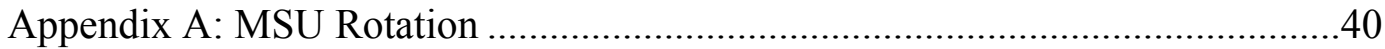

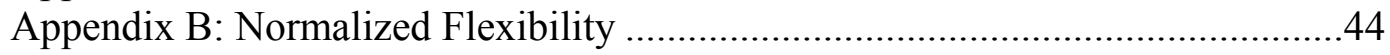

Appendix C: MSU Percent Change in Contribution.........................................46

Appendix D: Stiffness Curves ...................................................................50

VITA. 


\section{LIST OF TABLES}

Table 4.1 Average Global Rotations............................................................ 15

Table 5.1 Biomechanical Studies and Results at the Operative Level.....................26

Table A.1 MSU Rotation $\left(^{\circ}\right)$ Mean and Standard Deviation...................................43

Table B.1 Normalized Flexibility Statistics (ANOVA + SNK) $\quad$ N............................45

Table C.1 MSU Percent Change in Contribution Statistics

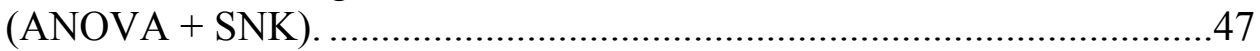




\section{LIST OF FIGURES}

Figure 2.1 Click’X Spine System Pedicle Screw Fixation..........................................3

Figure 2.2 Current Lumbar Disc Replacements ..................................................

Figure 3.1 Anterior Views of Implanted Spine Conditions....................................8

Figure 3.2 Differences in Lumbar Disc Replacement Devices ................................

Figure 3.3 Example of Determining Center of Rotation Differences with Fluoroscopic Imaging ............................................................11

Figure $3.4 \quad$ Lateral Bending Test Setup...............................................................11

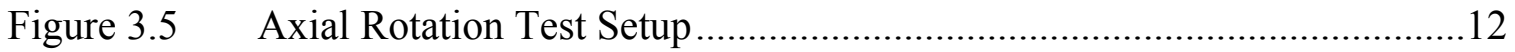

Figure 4.1 MSU Rotations of the Different Spine Conditions for Flexion and Extension...................................................................... 16

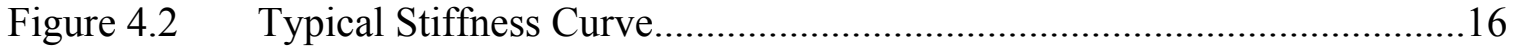

Figure 4.3 Normalized Flexibility in Flexion and Extension .................................17

Figure 4.4 Normalized Flexibility in Left and Right Lateral ..................................18

Figure 4.5 Normalized Flexibility in Left and Right Axial...................................19

Figure 4.6 Resultant MSU Percent Change in Contribution of Constructs during Flexion and Extension

Figure 4.7 Resultant MSU Percent Change in Contribution of Constructs during Left and Right Lateral

Figure 4.8 Resultant MSU Percent Change in Contribution of Constructs during Left and Right Axial Rotation

Figure A.1 MSU Rotations of the Different Spine Conditions for Left and Right Lateral

Figure A.2 MSU Rotations of the Different Spine Conditions for Left and Right Axial

Figure D.1 Harvested Extension-Stiffness Curves. 
Figure D.2 ProDisc-L Extension—Stiffness Curves.............................................52

Figure D.3 Maverick Extension-Stiffness Curves ...............................................53

Figure D.4 Pedicle Screw Fixation Extension—Stiffness Curves ..............................54

Figure D.5 Harvested Flexion-Stiffness Curves ...............................................55

Figure D.6 ProDisc-L Flexion—Stiffness Curves ...............................................56

Figure D.7 Maverick Flexion—Stiffness Curves...................................................57

Figure D.8 Pedicle Screw Fixation Flexion—Stiffness Curves .................................58

Figure D.9 Harvested Left Lateral—Stiffness Curves .........................................59

Figure D.10 ProDisc-L Left Lateral—Stiffness Curves..........................................60

Figure D.11 Maverick Left Lateral—Stiffness Curves ........................................61

Figure D.12 Pedicle Screw Fixation Left Lateral—Stiffness Curves ..........................62

Figure D.13 Harvested Right Lateral—Stiffness Curves.........................................63

Figure D.14 ProDisc-L Right Lateral—Stiffness Curves ..........................................64

Figure D.15 Maverick Right Lateral—Stiffness Curves.........................................65

Figure D.16 Pedicle Screw Fixation Right Lateral—Stiffness Curves ........................66

Figure D.17 Harvested Left Axial—Stiffness Curves ..........................................67

Figure D.18 ProDisc-L Left Axial—Stiffness Curves ............................................68

Figure D.19 Maverick Left Axial—Stiffness Curves .............................................69

Figure D.20 Pedicle Screw Fixation Left Axial—Stiffness Curves............................70

Figure D.21 Harvested Right Axial—Stiffness Curves ........................................ 71

Figure D.22 ProDisc-L Right Axial—Stiffness Curves...........................................72

Figure D.23 Maverick Right Axial—Stiffness Curves ........................................73

Figure D.24 Pedicle Screw Fixation Right Axial—Stiffness Curves ..........................74 


\section{CHAPTER 1: INTRODUCTION}

The incidence of lower back pain is high in the United States. It is estimated that more than seventy percent of the population experiences a significant episode of lower back pain. Identification of the pain source is not always clear and may be the result of multiple deficiencies. Degenerative disc disease is one of the major indications that leads to pain (1). If conservative treatments of degenerative disc disease are not successful, potential candidates for surgical procedures are screened. The most common surgical procedure is some form of fusion, whereby the motion of the problematic joint is drastically reduced. The efficacy of fusion has been in question for decades including questions of adjacent level degeneration because of motion redistribution (2-5).

Newer surgical techniques seek to improve upon fusion outcomes by preserving or restoring motion. One of the major classes of non-fusion surgery is lumbar disc replacement. It is a growing field and only recently has become available to the United States market. Better implant designs will emerge as the field of disc replacement grows and more results are obtained in both in vivo and in vitro studies. In vitro studies offer a controlled environment to describe disc replacement characteristics. Conclusions derived from in vitro results are also dependent on testing protocols. In older studies, the protocols fail in the ability to describe important issues such as adjacent level change in motion contribution (6). Even when supposedly using the same protocol, replication of results has been inconsistent. Head to head studies, either in vivo or in vitro, are rare. Studying two disc devices in the same setting is valuable for reducing errors and enhancing comparisons. The current study holds a unique advantage for these reasons.

The objective was to differentiate two new disc replacement systems in a relevant in vitro environment. To determine preservation abilities of ProDisc- $\mathrm{L}$ and Maverick lumbar disc prostheses, comparisons are normalized to the first tested harvested spine condition. Fusion is still a competitor of disc replacement and a pedicle screw fixation served as a negative control. Different results between ProDisc-L and Maverick were attributable to differences in geometric design specification. 


\section{CHAPTER 2: BACKGROUND}

\section{Anatomy of the Spine}

The lumbar spine is divided into five motion segments starting from the top at L1L2 down to L5-S1. Lumbar spinal structures and their general functions have been identified for quite some time. However, full kinematics and loading of the lumbar spine are still being investigated. Disc replacement systems will likely affect three major anatomical spinal features.

The first structure is the intervertebral disc itself. An asymptomatic intervertebral disc in the lumbar spine is able to withstand high compressive loads due to the nucleus pulposus. The disc also has an annulus fibrosus layer capable of restricting all directional motion. Intervertebral discs undergo inevitable loss in function over time. When a disc shows mark decline in functionality, it is given the term degenerative disc disease. Why some patients will have more pain than others is unknown. Practitioners vary in their stance on what constitutes normal degeneration and where it crosses over to disease categorization. Removal of the degenerate disc is central to all surgical techniques.

The second structures are the two facet joints found on every motion segment. Lumbar facet joints prevent over-extension but are less involved in flexion. The facets are also resistant to anterior shear loads, but not posterior (7). Facet joint pain may arise independent from degenerative disc disease (8) or as a continuation of disc degeneration (9-11).

The third structure is the anterior longitudinal ligament. This ligament provides stability in extension. Running down the anterior face of the motion segment, its functionality is lost during the disc replacement surgery. Cutting the anterior longitudinal ligament at the operative level means the loss of its functionality for up to four of the surrounding vertebrae (12).

\section{Lumbar Fusion}

Lumbar spinal fusion represents a radical alteration to biomechanics. The drastic reduction of motion at the problematic joint is rarely seen in other joints, i.e., hip, knees. With over 200,000 spinal fusions performed yearly, it is seen as a common and viable surgical approach. Pedicle screw fixation systems such as Click'X show in Figure 2.1 are becoming increasingly popular in fusion surgeries. Reports of lumbar fusion operations typically achieve success rates ranging from 74-90\% (13). However, there are generally four complaints concerning fusion of the lumbar spine: 1) Pain relief is not guaranteed, 2) difficulty in achieving fusion, 3 ) potential of creating a non-physiological sagittal balance, and 4) adjacent level degeneration $(2,4)$. 


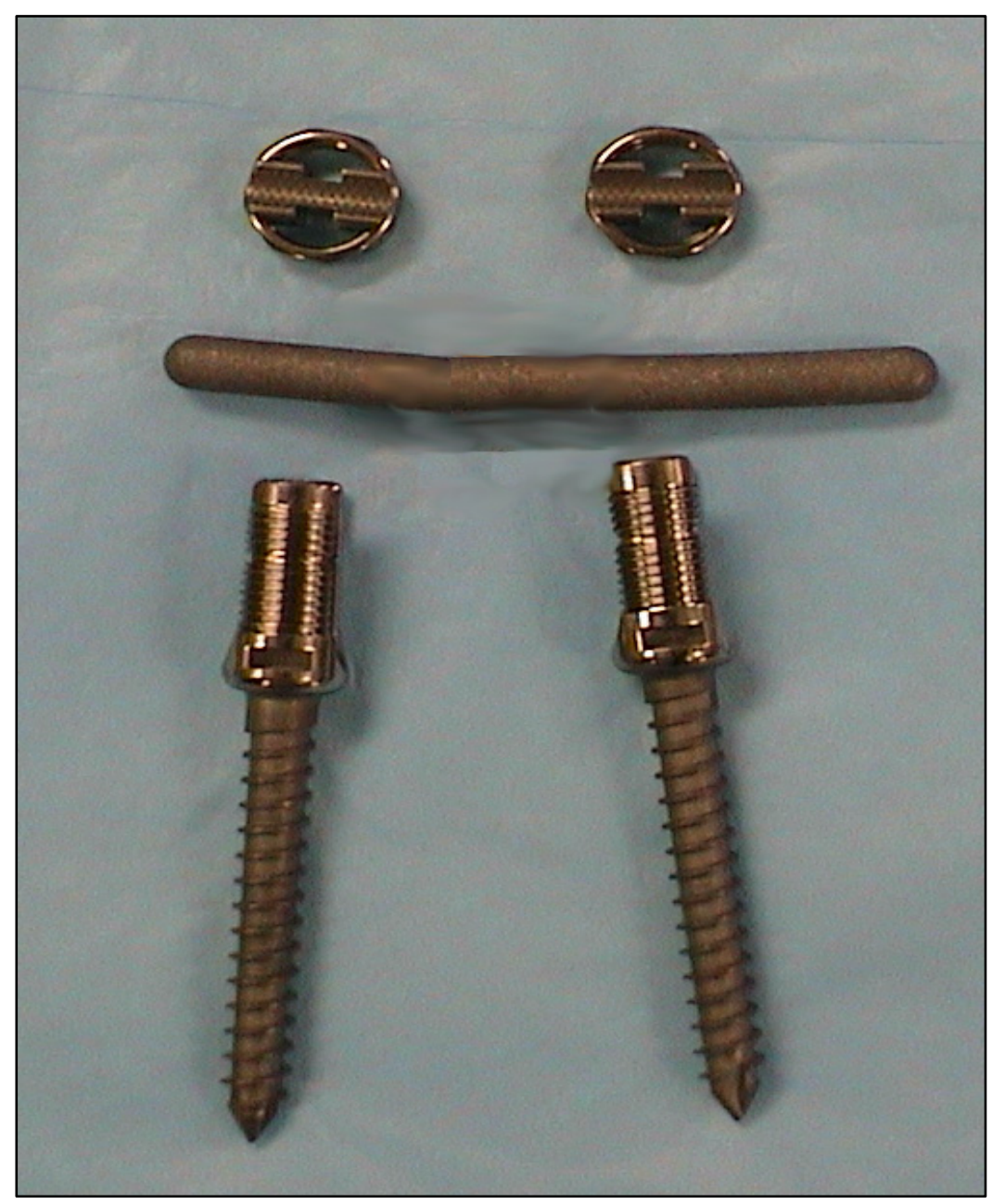

Figure 2.1: Click'X Spine System Pedicle Screw Fixation. 
Studies have shown a disconnect between radiographic success and pain reduction (14). Part of the problem is defining proper indications for fusion. By narrowing patient selection, successful pain relief rises (15). The second issue concerns the difficulty of bone healing or pseudoarthrosis. During surgery attempts are usually made to increase or maintain lordosis. Whatever position is chosen to be most comparable to asymptomatic condition will be permanently adopted by the patient. If the balance is abnormal, other spinal structures experience abnormal loading leading, which leads to further wear problems. Finally, the issue of adjacent level degeneration lies in the idea that reduction of motion at the fused level leads to a redistribution of loading such that other motion levels degenerate in quality.

Current advancements in spinal fusion are aimed at less intrusion on muscles, joints, and ligaments in hopes of decreasing patient recovery time and increasing chances of success. Recent advancements in the area of fusion involve the use of minimally invasive techniques. Various newer techniques exist, including TLIF, PLIF, and ALIF (retroperitoneal and transperitoneal). Pedicle screw fixation may be used in conjunction with any of the above methods. Ozgur et al have a technique called extreme lateral interbody fusion (XLIF), in which a minimally disruptive lateral retroperitoneal approach is utilized (16). The axial lumbar interbody fusion (AxiaLIF) has been performed for L5$\mathrm{S} 1$ fusion and requires only a $2 \mathrm{~cm}$ incision near the tailbone (17). Initial results have indicated shorter recovery times but not necessarily a difference in long term patient outcome (18).

\section{Artificial Disc Replacement}

Disc replacements attempt to rectify the shortcomings mentioned in association with fusion. The prosthesis is intended to replace the nucleus pulposus, portions of the annulus fibrosus, and ,sometimes, the vertebral end plates. The time and difficulty to fuse joints is no longer required in disc replacements. At least in the short term, patient satisfaction is higher due to the lower recuperative time $(19,20)$. Height restoration and mobility of the disc should allow the patient to adopt a natural sagittal balance. Unlike the fixed alignment found from fusion, the surgeon would have more leeway with the disc replacement. It is believed that by allowing motion similar to asymptomatic conditions, other levels will not become overstressed and result in adjacent level degeneration.

Several artificial disc replacements have been presented in the past but currently three are available or soon to be available for general patient implantation in the United States. Charité lumbar disc prosthesis (DePuy Spine, Warsaw, IN) was the first to gain approval from the US Food and Drug Administration in 2004 (Figure 2.2A). There are more than 7000 implanted patients. The device features a mobile polyethylene core and endplates with small teeth that provide vertebral fixation. The ProDisc-L lumbar disc prosthesis (Synthes Spine, West Chester, PA) entered the US market in 2006 with more than 2300 ProDisc-L devices implanted worldwide (Figure 2.2B). The ProDisc-L implant is materially similar to Charité disc but with a fixed center of rotation. The third disc replacement is the Maverick lumbar disc prosthesis (Medtronic Inc., Memphis, TN) and 


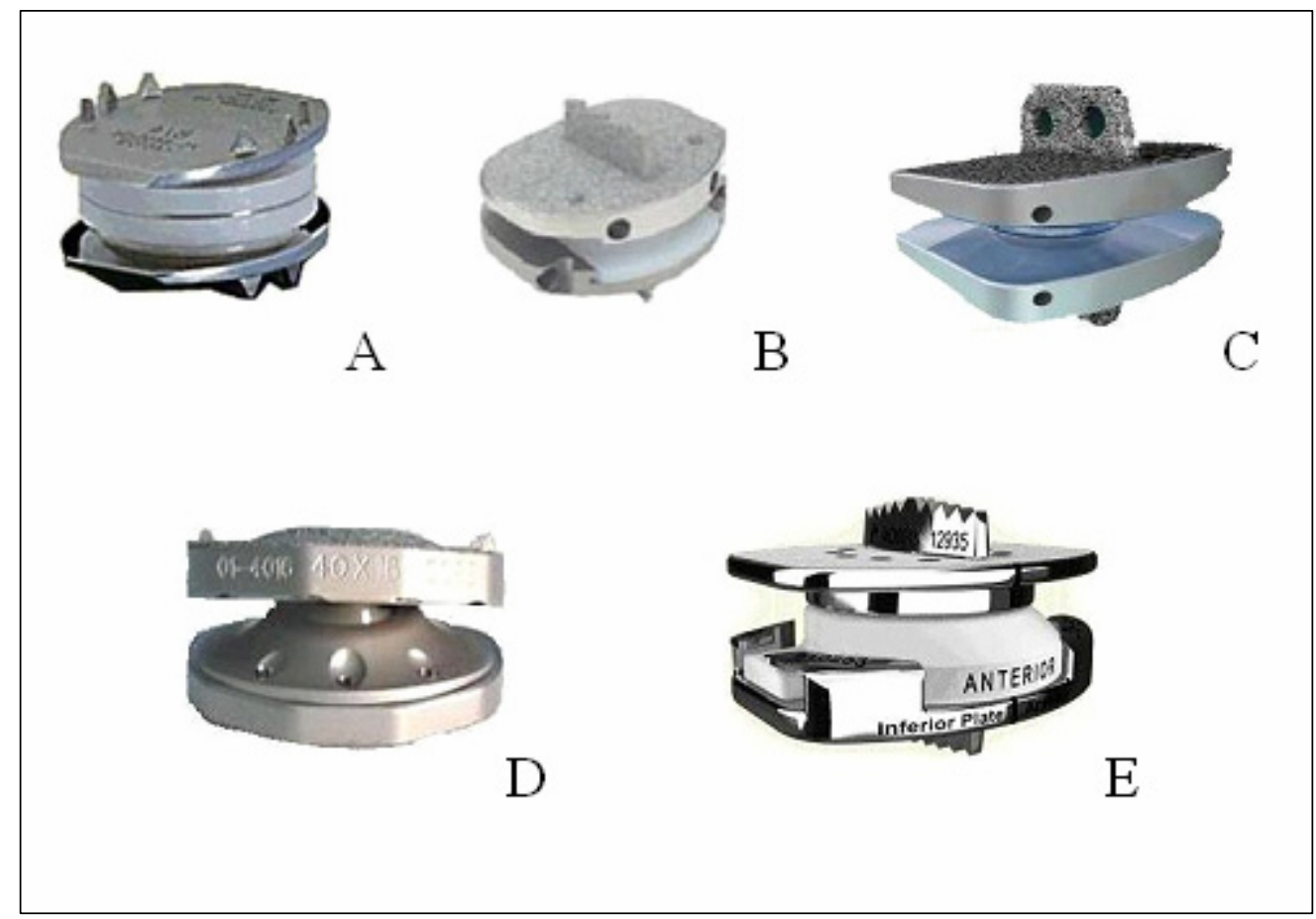

Figure 2.2: Current Lumbar Disc Replacements. These include A) Charité, B) ProDisc-L, C) Maverick, D) FlexiCore, and E) Mobidisc. 
is expected to be the next to enter the US market (Figure 2.2C). The Maverick disc has the same general ball and socket design as ProDisc-L implant but features a metal-onmetal joint. Further from approval are the Flexicore and Mobidisc disc implants. The Flexicore (Stryker Corp., Kalamazoo, MI) features a fixed center of rotation along with metal on metal contact like the Maverick disc, but unlike Charité, ProDisc-L, and Maverick discs, it is limited in its axial rotation (Figure 2.2D). Finally the Mobidisc (LDR Spine, Austin, TX) attempts to more closely replicate the center of rotation by allowing translation similar to the Charité disc (Figure 2.2E). 


\section{CHAPTER 3: MATERIALS AND METHOD}

\section{Specimen Preparation}

Seven asymptomatic human cadaveric lumbar spines were used in the study. The average age was $43 \pm 7$ years. Tissue procurement and evaluation were done using a previously established protocol (21-23). The L1 vertebral body and the sacrum were mounted into cylindrical pots that positioned the specimen in a neutral upright orientation. A low-melting-point bismuth alloy (Small Parts, Miami Lakes, FL) provided final fixation. Threaded rods through the vertebral bodies secured light emitting diode (LED) targets used with the motion tracking system. Rods and targets were positioned or repositioned as to not interfere with instrument insertion or device performance.

The spines were mounted in a programmable testing apparatus and nondestructively tested in the intact harvested condition. After testing the harvested condition, a discectomy at L5-S1 followed by insertion of ProDisc-L was performed by a spine surgeon (A. Sin, MD). The spine surgeon recommended the same size discs (M) for all specimens (Figure 3.1A). After the ProDisc-L implanted spines were tested, the discs were extracted and another spine surgeon (R. Bertagnoli, MD) implanted the Maverick disc, as per manufacturer's specifications (Figure 3.1B). In total, four different spine conditions were studied: 1) Harvested, 2) L5-S1 discectomy and implantation of the ProDisc-L disc, 3) removal of the ProDisc-L device and implantation of the Maverick disc at L5-S1, and 4) Posterior pedicle screw fixation at L5-S1 with a Maverick left in the disc space.

The Click X pedicle screw fixation system (Synthes Spine) was used to simulate a fused spine condition. For the fused spine conditions, the discs implants were left in the specimen as a rigid spacer. All surgeries were performed by a spine surgeon (A. Sin, $\mathrm{MD})$ under fluoroscopic guidance at the Medical Education Research Institute (Memphis, TN).

\section{Summary of Instrumentation Notes}

All seven specimens were implanted with the same sized (medium) ProDisc-L and Maverick devices (Figure 3.1). The outer dimensions of both disc replacement devices were nearly identical: the A-P depths were both $27 \mathrm{~mm}$, heights were both 12 $\mathrm{mm}$, and ProDisc-L's width was $34.5 \mathrm{~mm}$ versus Maverick's width of $35 \mathrm{~mm}$. The two different ball-and-socket type implants were designed to reside at different places within the disc itself. The ProDisc-L disc was centered on the posterior device while the ball and socket on Maverick is located $5 \mathrm{~mm}$ further posterior (Figure 3.2C and Figure 3.2D). For the ProDisc-L implant, the distance between the center of rotation of the disc and the posterior longitudinal ligament measured on fluoroscopic images, was $16.70 \pm 1.83 \mathrm{~mm}$, while the same measurement for the Maverick implanted spines was $11.95 \pm 1.52 \mathrm{~mm}$ 


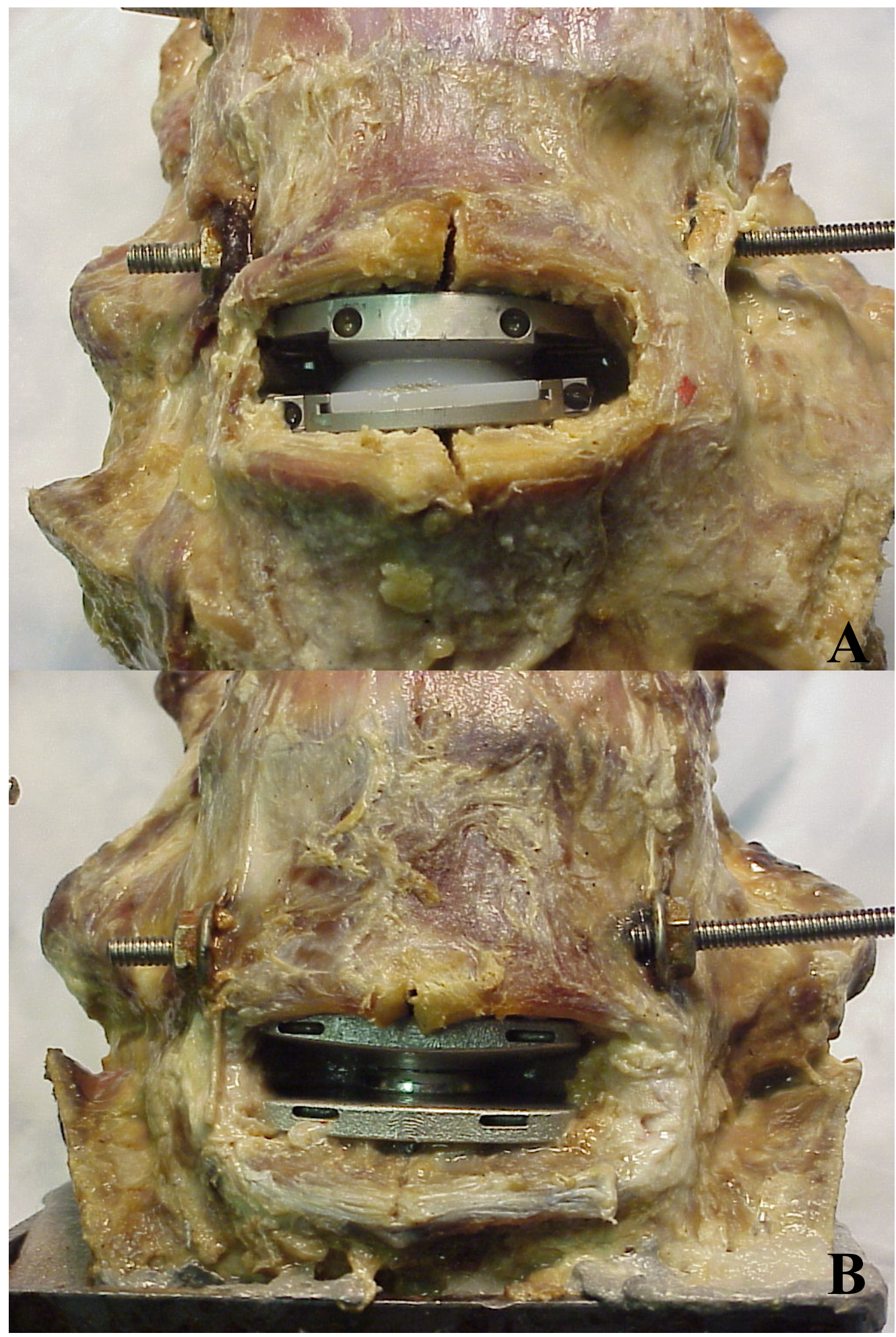

Figure 3.1: Anterior Views of Implanted Spine Conditions. A) ProDisc-L Disc and B) Maverick Disc. 

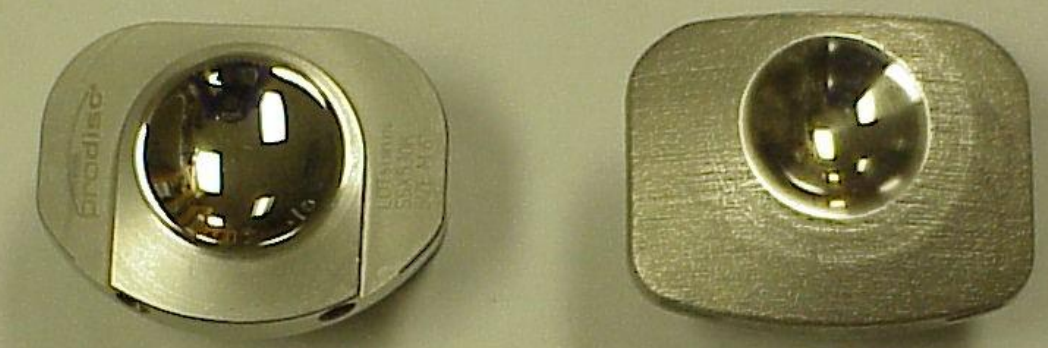

A

B

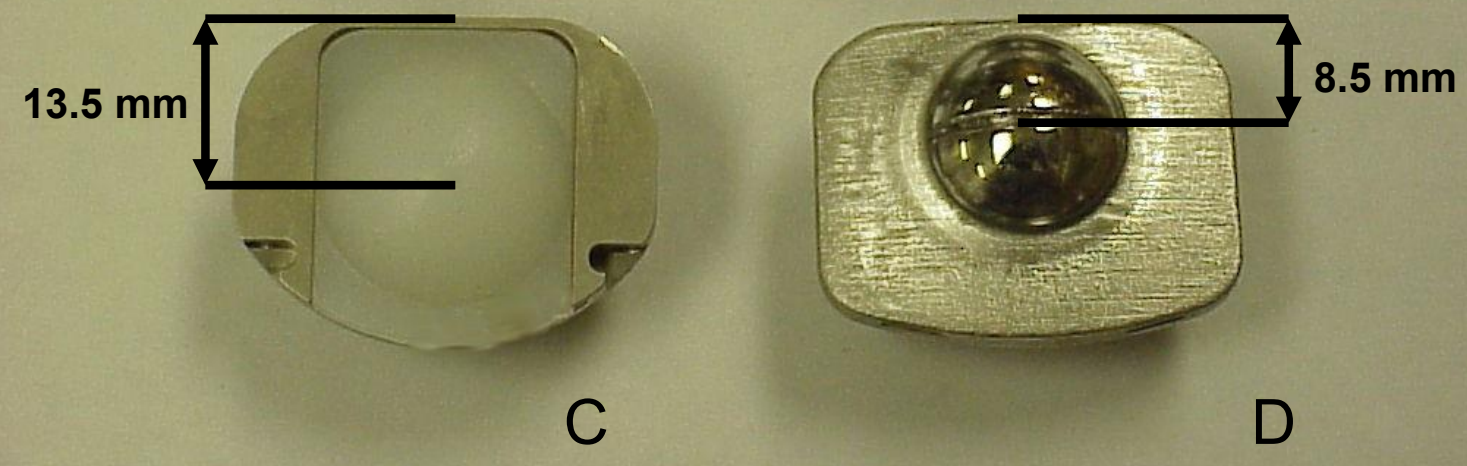

Figure 3.2: Differences in Lumbar Disc Replacement Devices. A) Superior ProDisc-L endplate. B) Superior Maverick endplate. C) Inferior ProDisc-L endplate. D) Inferior Maverick endplate. 
(Figure 3.3). Another design feature that was different between the two disc implants was the size of the radius of curvature of the ball component. The ProDisc-L implant was approximately $9 \mathrm{~mm}$ and the Maverick disc had a radius of curvature of $8.1 \mathrm{~mm}$. The ProDisc-L device was also reported to allow $20^{\circ}$ in flexion-extension and lateral bending compared to $16^{\circ}$ for the Maverick disc (24).

\section{Nondestructive Testing Protocol}

All biomechanical tests were performed in the Joint Implant Biomechanics Laboratory in the Department of Biomedical Engineering and Imaging at UTHSC, Memphis, TN. An existing testing protocol was used to test the spines in flexion/extension, right and left lateral bending, and right and left axial rotation $(25,26)$. Since axial rotation is strongly coupled with lateral bending, the spines were left unconstrained in axial rotational during the lateral bending tests (Figure 3.4). All tests were performed under displacement control with the spine positioned at a $200 \mathrm{~mm}$ offset distance from the actuator load axis. The spinal constructs were mounted in a biomechanical testing apparatus programmed to output a triangular shaped displacementtime waveform of $6.4 \mathrm{~mm} / \mathrm{sec}$, corresponding to approximately $2.0 \mathrm{deg} / \mathrm{sec}$ overall spine motion.

Each spine condition was tested in flexion, extension, lateral bending, and axial rotation each up to a target moment of $8 \mathrm{Nm}$, or stopped if the axial force exceeded $200 \mathrm{~N}$ or a maximum global rotation of $30^{\circ}$ occurred. For axial rotational tests, a $100 \mathrm{~N}$ compressive load was applied to the spinal construct (Figure 3.5). Each test trial included three loading cycles and data from the second cycle were analyzed.

\section{Data Management and Analysis}

Measurements included movement of individual vertebral bodies, global spine motion, and global loads applied to the spine. Global rotation and applied load data were used to calculate overall spinal stiffness and flexibility. Variations in the motion measurements were analyzed at an end limit of global moment common to all spine conditions within each specimen. To account for intrinsic differences in specimen tissue quality, all instrumented spine conditions were normalized with respect to their harvested condition. Motion responses at each operated motion segment unit (MSU) were normalized by first expressing the individual segment motion relative to the global spinal rotation for the instrumented spine condition ( (L5-S rotation of operated spine)/( L1-S rotation of operated spine) ) and then dividing by the same ratio for the intact spine condition ( (L5-S rotation of intact spine)/( L1-S rotation of intact spine) ). The same normalization procedure was used to compare percent changes in MSU rotations at the remaining non-operated spinal levels for the instrumented spine conditions. 

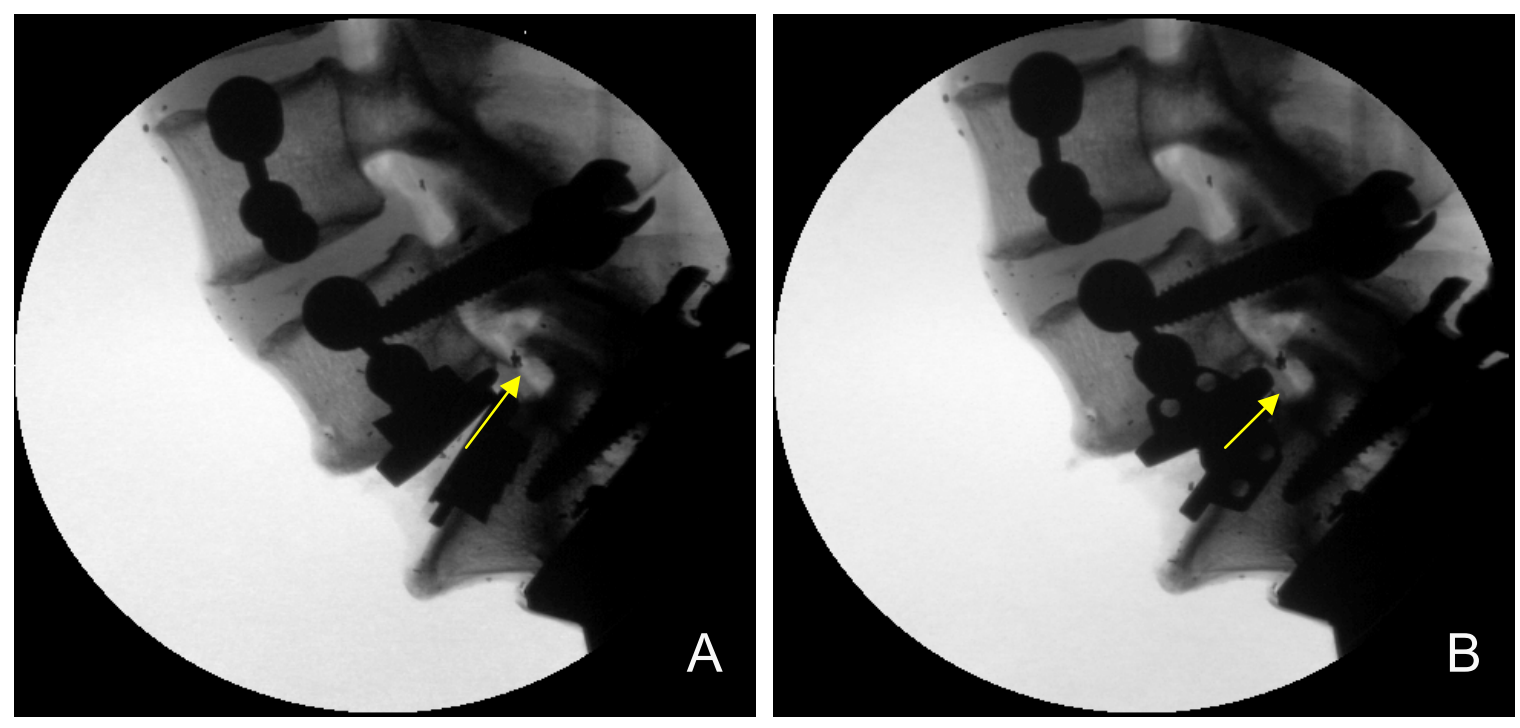

Figure 3.3: Example of Determining Center of Rotation Differences with Fluoroscopic Imaging. Measurements made from ball and socket center to posterior longitudinal ligament. A) ProDisc-L implant and B) Maverick disc.

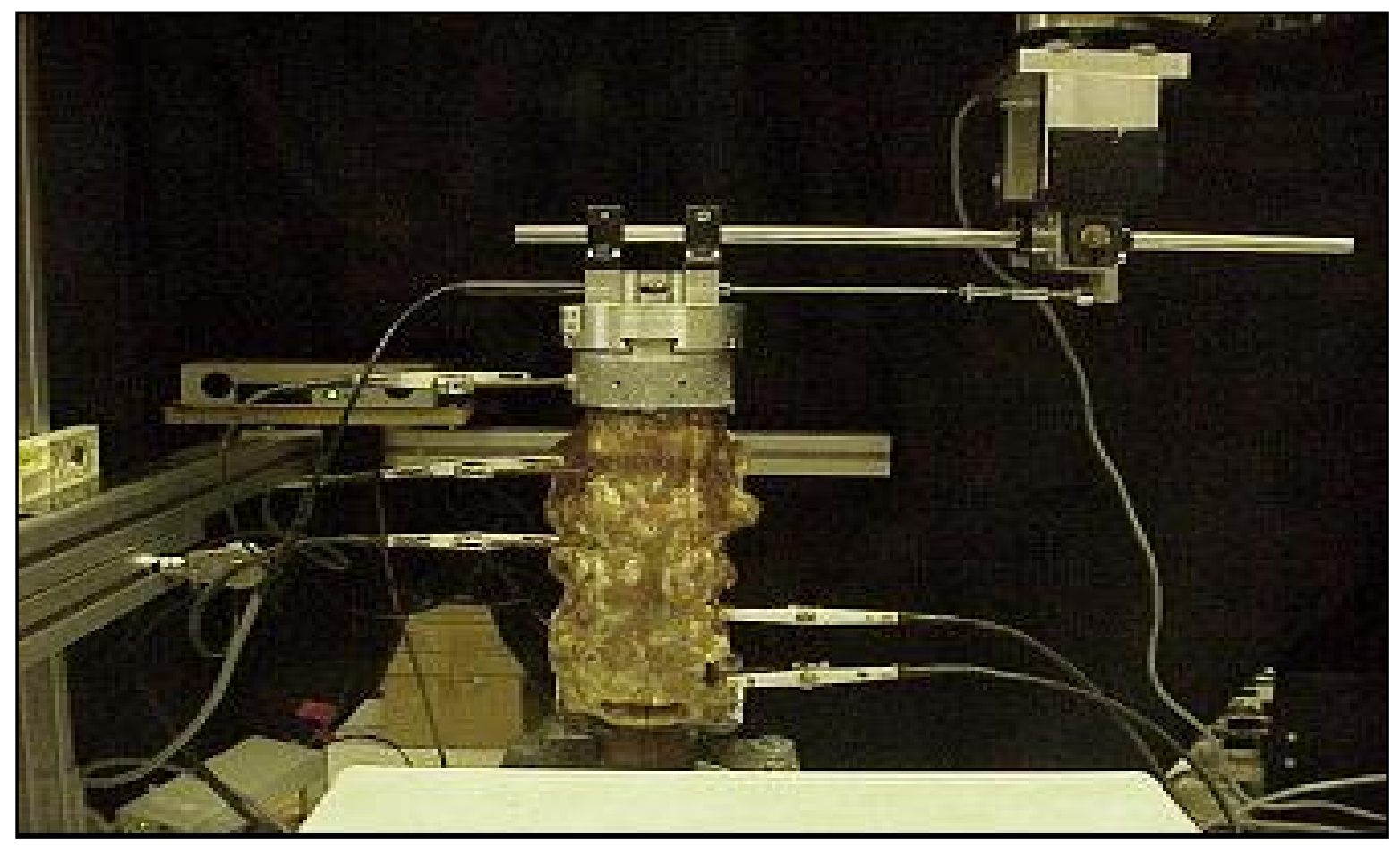

Figure 3.4: Lateral Bending Test Setup. 


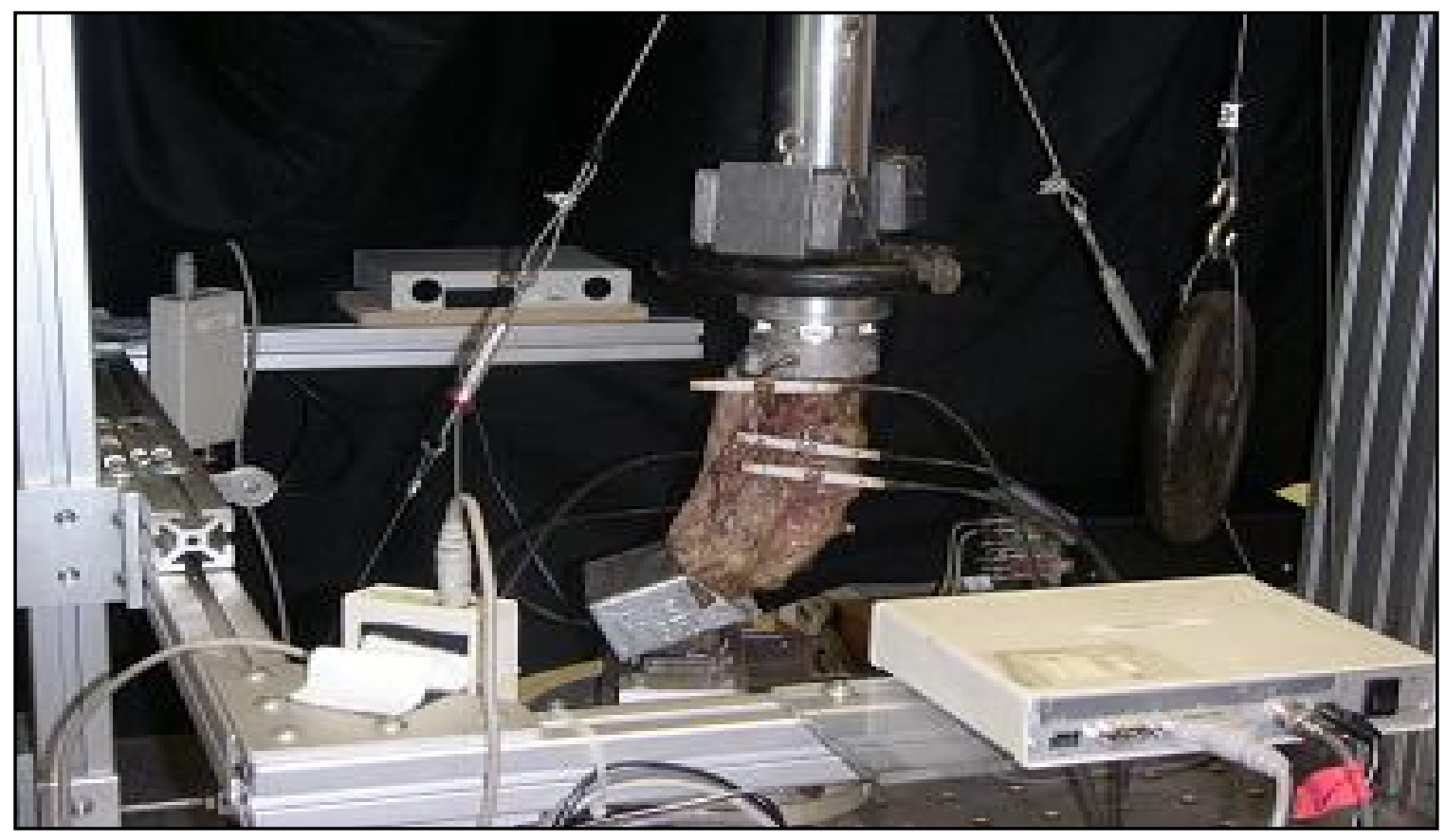

Figure 3.5: Axial Rotation Test Setup. 
Flexibility $\left({ }^{\circ} / \mathrm{Nm}\right)$ is calculated by dividing the global rotation by the corresponding induced moment. Normalized flexibility is achieved by dividing an instrumented specimen's flexibility with its harvested result (Equation 3.1).

$\begin{aligned} & \text { Normalized } \\ & \text { Flexibility }\end{aligned}=\frac{\begin{array}{cc}\text { Global Rotation (degrees) } \\ \text { Observed Moment }(\mathrm{Nm})\end{array}}{\begin{array}{c}\text { (Instrumented Spine } \\ \text { Condition ) }\end{array}} \begin{gathered}\text { (Harvested Spine } \\ \text { Condition) }\end{gathered}$

Motion at each level is calculated as percent contributions towards the overall rotation. The percent change in contribution is the normalized value of the instrumented condition using the harvested values. Equation 3.2 is used for all 5 levels for each specimen in order to calculate percent change in contribution.

\begin{tabular}{|c|c|c|c|}
\hline \multirow{2}{*}{$\begin{array}{l}\text { Percent Change } \\
\text { in Motion } \\
\text { Contribution }\end{array}$} & & $\frac{\text { Individual Rotation (MSU) }}{\text { Total Rotation (L1-S1) }}$ & $\begin{array}{l}\text { (Instrumented Spine } \\
\text { Condition) }\end{array}$ \\
\hline & $=$ & $\frac{\text { Individual Roation (MSU) }}{\text { Total Rotation (L1-S1) }}$ & $\begin{array}{l}\text { (Harvested Spine } \\
\text { Condition) }\end{array}$ \\
\hline
\end{tabular}

Flexibility and normalized motion data were compared using one way repeated measure ANOVA, followed by a Student Newman Keuls (SNK) test for pairwise comparison when a significant difference among the groups was detected by the ANOVA. Significance was defined as a $\mathrm{p} \leq 0.05$. 


\section{CHAPTER 4: RESULTS}

\section{MSU Rotations}

The average global rotation of the different spine conditions for the different loading modes is listed in Table 4.1. The average moments obtained were below the targeted $8 \mathrm{Nm}$ for all modes, ranging from 7.2 (right lateral bending) to 7.7 (left axial rotation). As an example, the mean MSU rotations at each spinal level are shown in Figure 4.1 for the four spine conditions under flexion and extension. MSU rotation figures for lateral bending and axial rotation are located in Appendix A.

\section{Global Stiffness/Normalized Flexibility}

The global moment and rotation performance of each specimen can be most easily observed via stiffness curves. Proper loading and unloading of specimens can be identified by charting these curves. Physiological displays of hysteresis, tissue deficiencies, and faulty testing sequences can all be identified before further analysis is performed. An example of a typical stiffness curve is given in Figure 4.2 and the remaining stiffness curves appear in Appendix B. The inverse of stiffness represents the flexibility of the spinal construct and is discussed next. Average values of normalized flexibility for flexion and extension loading are shown in Figure 4.3. Values below 1 indicate a loss in flexibility as compared to the harvested condition. The ProDisc-L and pedicle fixation conditions were significantly less flexible than the harvested spine condition in flexion. The Maverick implanted condition was not significantly different from the harvested condition but more flexible than both ProDisc-L and pedicle screw fixation conditions. In extension, both disc replacement groups were significantly more flexible than the harvested spine conditions.

ProDisc-L, Maverick and fixation did not significantly alter lateral bending (Figure 4.4) or axial rotation flexibility (Figure 4.5). Flexibility of Maverick is higher on average in all loading modes but only significantly in flexion and extension. This is due to the imposed $30^{\circ}$ global rotation limit. The values for normalized flexibility and the statistical analyses are found in Appendix C.

\section{MSU Percent Change in Contribution}

Each spinal level's percent change in contribution was calculated using Equation 3.2. Analysis of MSU percent change in contribution consisted of combined motion in addition to the six directional tests: flexion, extension, right and left lateral bending, and right and left axial rotation. The numerical values of the MSU percent change in contribution along with the statistical summary are provided in Appendix D. 


\section{Table 4.1: Average Global Rotations.}

\begin{tabular}{lcccc}
\hline $\begin{array}{l}\text { Loading Mode } \\
(\text { Moment Nm) }\end{array}$ & Harvested & ProDisc-L & Maverick & Fixation \\
\hline Flexion (7.6) & $25.3 \pm 5.8$ & $16.4 \pm 6.0$ & $22.2 \pm 7.5$ & $14.1 \pm 6.7$ \\
Extension (7.6) & $12.9 \pm 5.7$ & $22.7 \pm 8.2$ & $24.3 \pm 8.9$ & $16.3 \pm 8.5$ \\
Left Lateral (7.3) & $19.0 \pm 3.9$ & $20.1 \pm 4.6$ & $22.7 \pm 4.4$ & $19.5 \pm 7.9$ \\
Right Lateral (7.2) & $17.2 \pm 4.3$ & $17.6 \pm 6.3$ & $19.5 \pm 4.8$ & $16.3 \pm 7.0$ \\
Left Axial (7.7) & $7.6 \pm 2.0$ & $6.0 \pm 1.6$ & $8.7 \pm 2.7$ & $6.1 \pm 2.4$ \\
Right Axial (7.6) & $7.1 \pm 1.8$ & $6.2 \pm 1.9$ & $8.8 \pm 2.7$ & $6.0 \pm 2.0$ \\
\hline
\end{tabular}



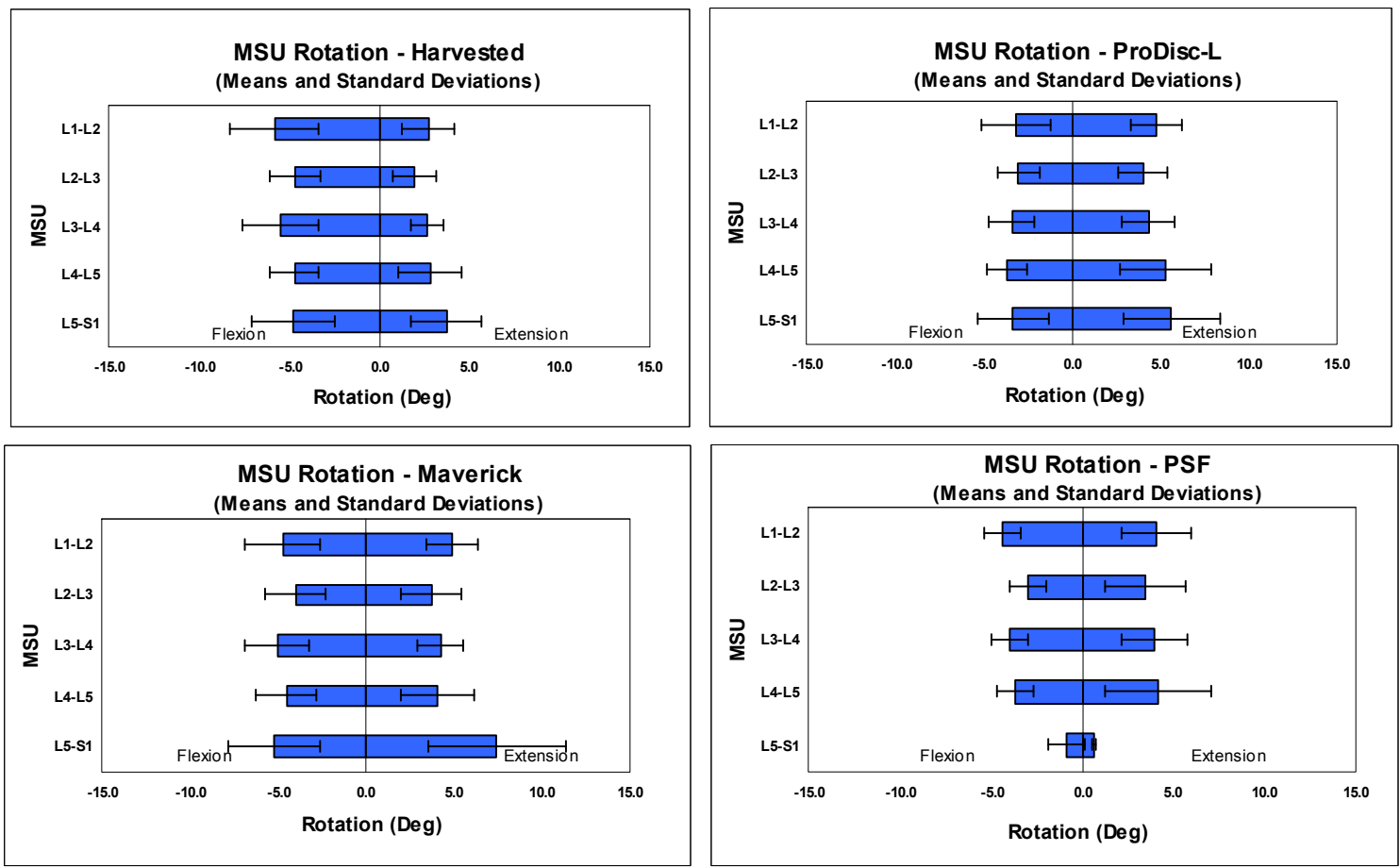

Figure 4.1: MSU Rotations of the Different Spine Conditions for Flexion and Extension.

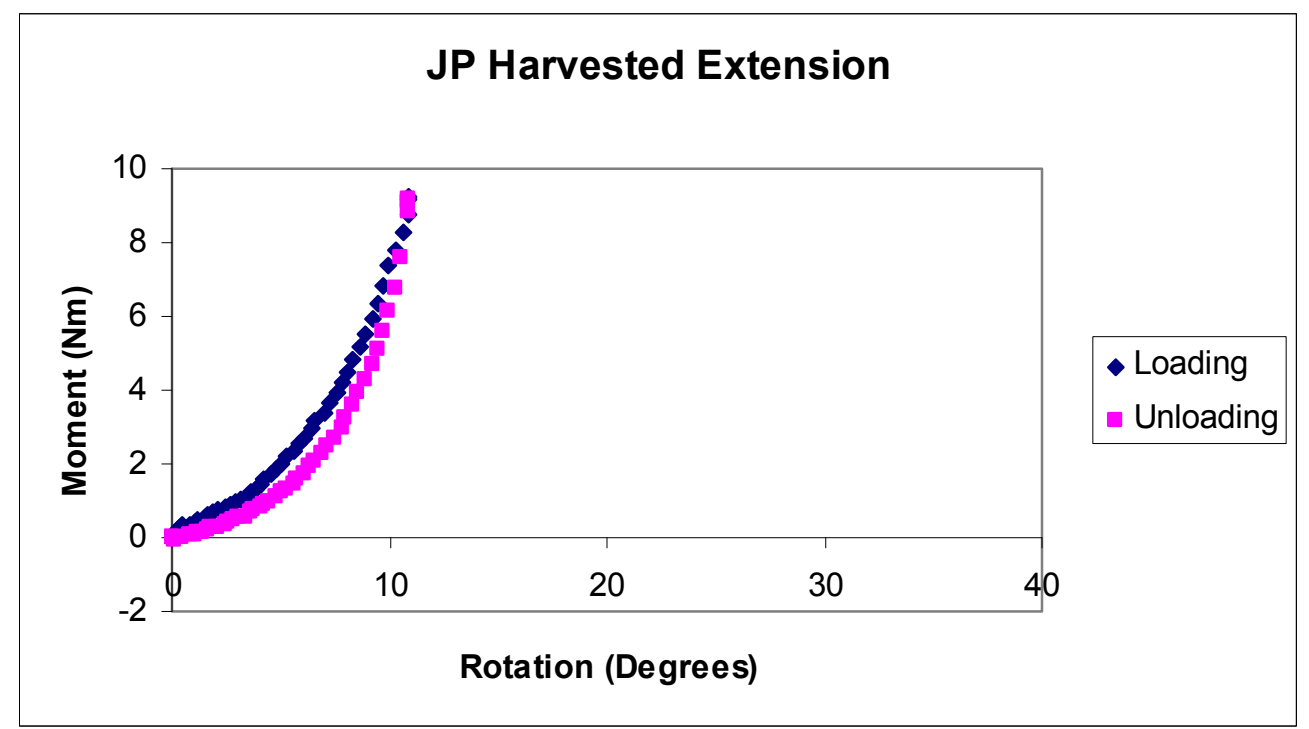

Figure 4.2: Typical Stiffness Curve. 

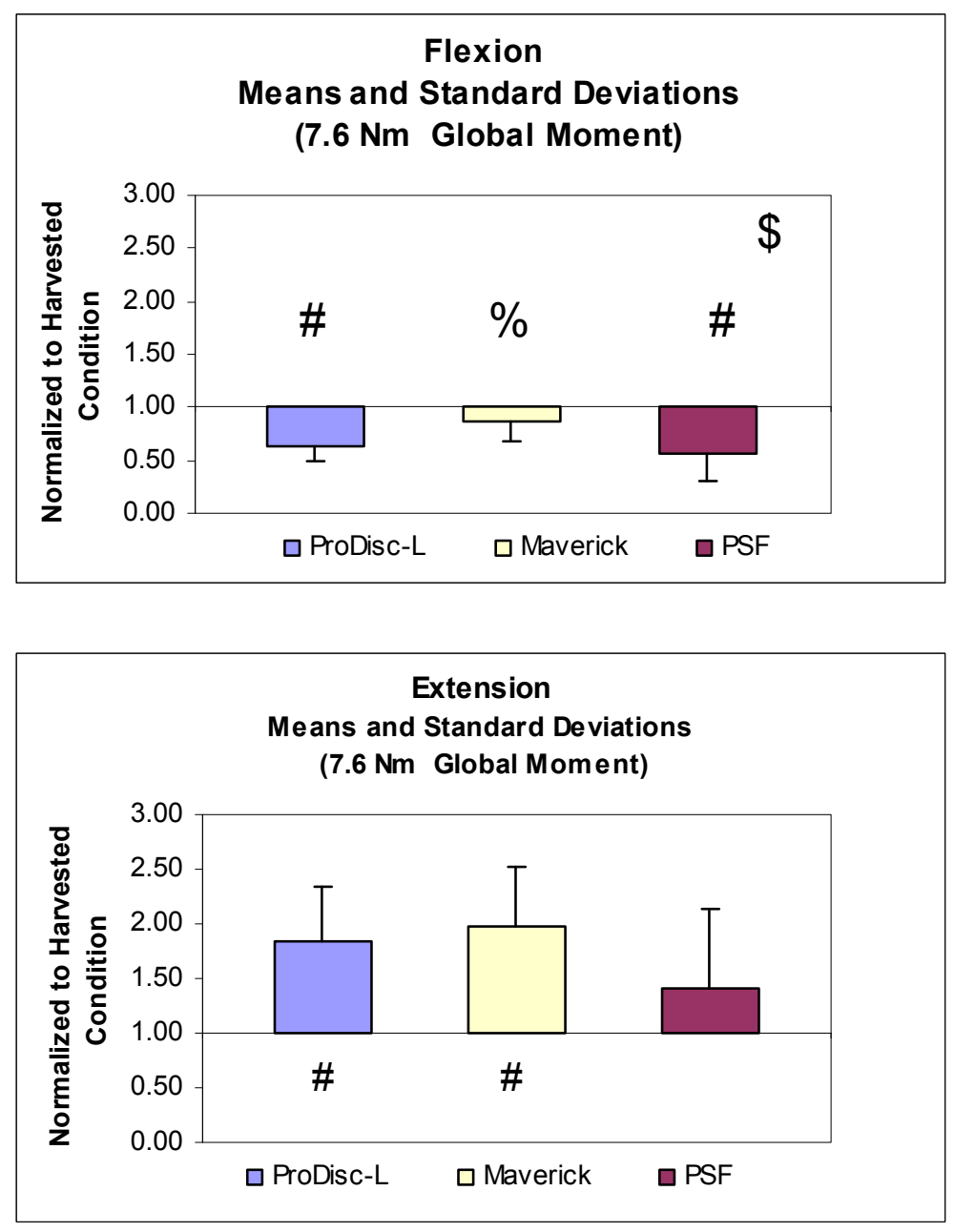

\begin{tabular}{cl}
\hline Symbol & Significant Difference From \\
$\#$ & Harvested (positive control) \\
$\%$ & Fixation (negative control) \\
$\$$ & Disc Replacements \\
\hline
\end{tabular}

Figure 4.3: Normalized Flexibility in Flexion and Extension. 

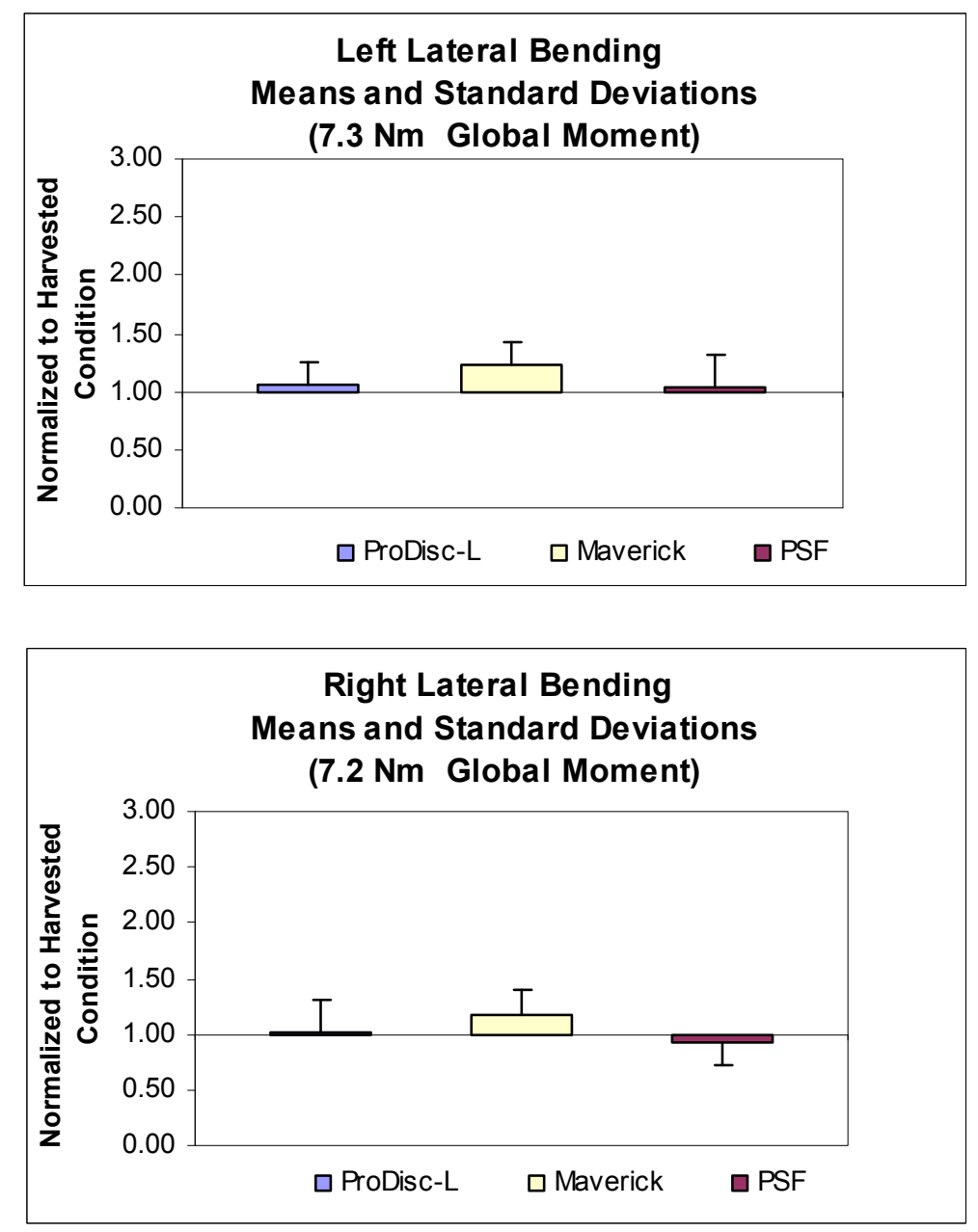

\footnotetext{
Significant Difference From

Harvested (positive control)

Fixation (negative control)

Disc Replacements
}

Figure 4.4: Normalized Flexibility in Left and Right Lateral. 

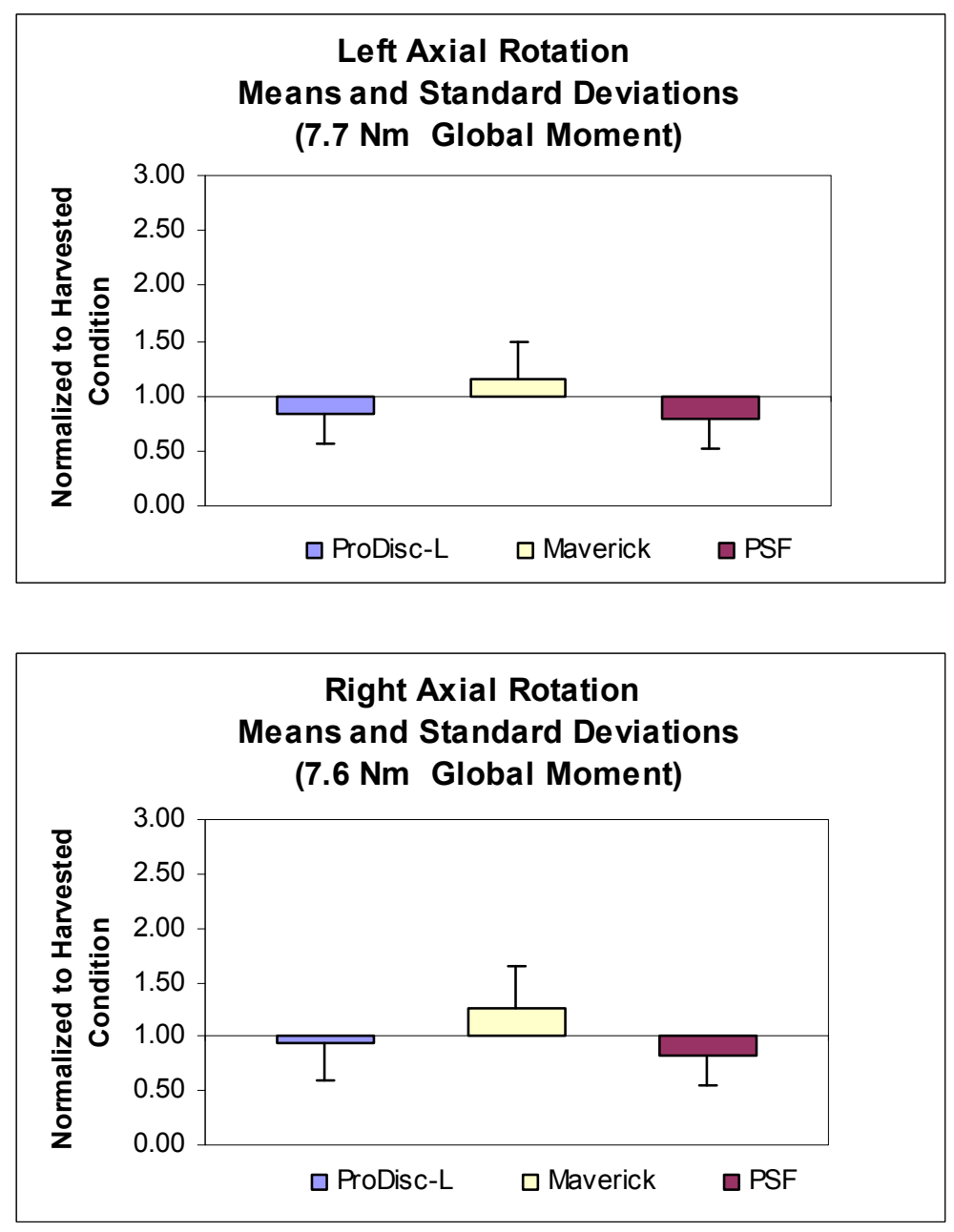

\begin{tabular}{cl}
\hline Symbol & Significant Difference From \\
$\#$ & Harvested (positive control) \\
$\%$ & Fixation (negative control) \\
$\$$ & Disc Replacements \\
\hline
\end{tabular}

Figure 4.5: Normalized Flexibility in Left and Right Axial. 
Changes in rotational distribution under flexion, extension, and combined flexion and extension are shown in Figure 4.6. There was no significant difference in the motion response at the operated between the ProDisc-L and the Maverick implanted conditions or between either implant condition and the harvested condition. However, significantly less motion occurred at the operated level of the pedicle screw fixation condition compared with the harvested condition and both implanted conditions during flexion, extension, and combined flexion plus extension. Significant differences only arise when looking at combined flexion and extension. L3-L4 contributed for pedicle screw fixation contributed significantly more motion than the ProDisc-L and Maverick implanted conditions. The L2-L3 level of pedicle screw fixation also significantly contributed more motion than the harvested condition.

Left and right lateral bending displayed different motion distributions within tests conditions themselves (Figure 4.7). At the operated level, right lateral bending for Maverick was significantly higher than that of pedicle screw fixation. The same statement is not true of left lateral bending for Maverick. When analyzing combined left and right lateral, no differences were detected for any of the test conditions.

During axial rotation, significantly less motion occurred at the operated L5-S1 level of the pedicle screw fixation spine condition compared to the ProDisc-L and Maverick implanted conditions (Figure 4.8). Although the adjacent levels of the pedicle screw fixation condition provided some compensation, the only significant increase occurred at L3-L4 compared to both implanted spine conditions. 

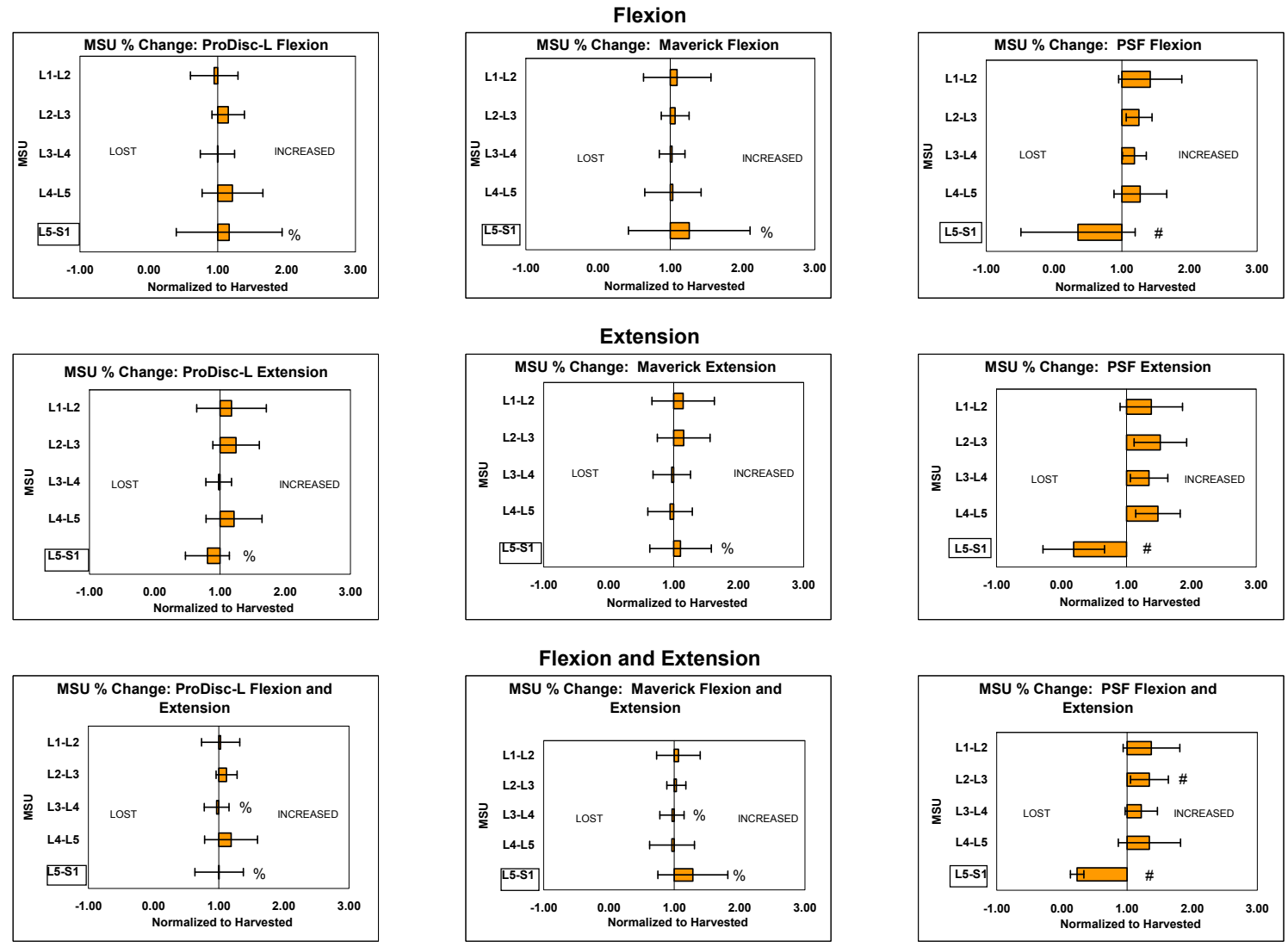

\begin{tabular}{cl}
\hline Symbol & Significant Difference From \\
$\#$ & Harvested (positive control) \\
$\%$ & Fixation (negative control) \\
$\$$ & Disc Replacements \\
\hline
\end{tabular}

Figure 4.6: Resultant MSU Percent Change in Contribution of Constructs during Flexion and Extension. 

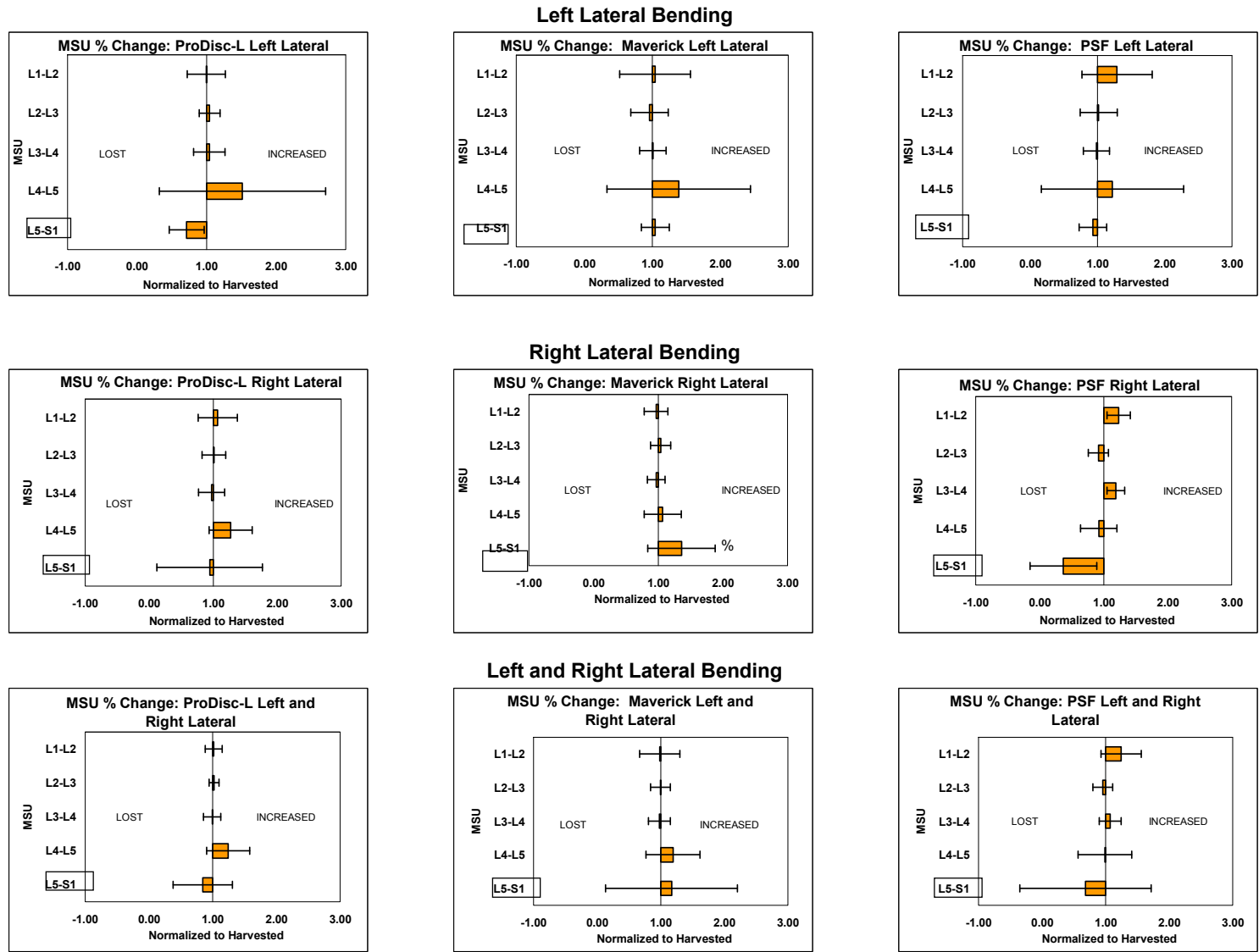

\begin{tabular}{cl}
\hline Symbol & Significant Difference From \\
$\#$ & Harvested (positive control) \\
$\%$ & Fixation (negative control) \\
$\$$ & Disc Replacements \\
\hline
\end{tabular}

Figure 4.7: Resultant MSU Percent Change in Contribution of Constructs during Left and Right Lateral. 


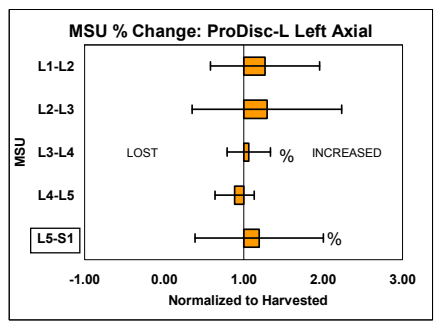

Left Axial Rotation
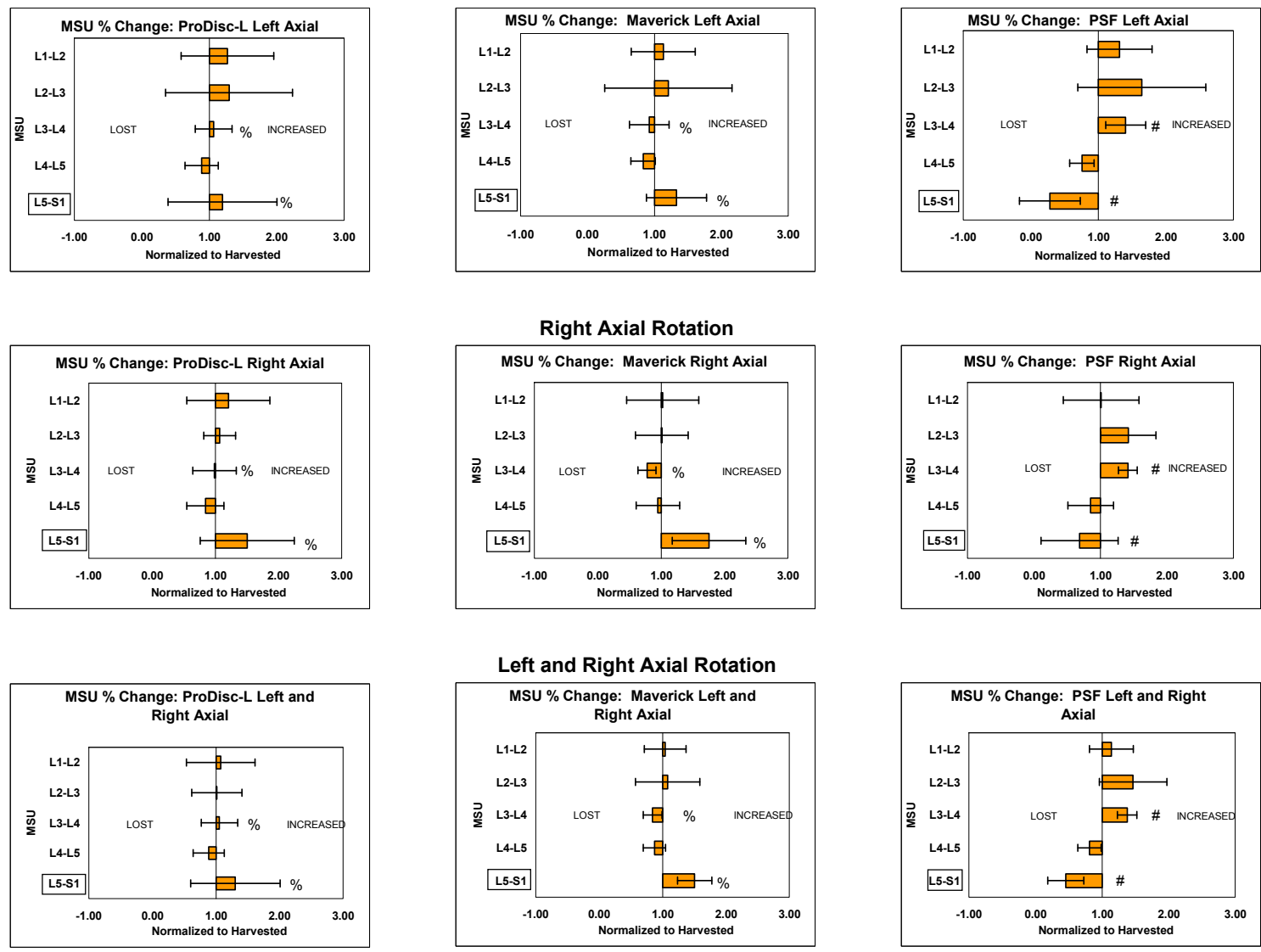

\begin{tabular}{cl}
\hline Symbol & Significant Difference From \\
$\#$ & Harvested (positive control) \\
$\%$ & Fixation (negative control) \\
$\$$ & Disc Replacements \\
\hline
\end{tabular}

Figure 4.8: Resultant MSU Percent Change in Contribution of Constructs during Left and Right Axial Rotation. 


\section{CHAPTER 5: DISCUSSION}

\section{Testing Protocol Differentiation}

No standardized testing methodology exists for in vitro tests using cadaveric tissue. As a result different biomechanical research groups have developed their own cadaver testing methods in attempts to best replicate in vivo motion. Consequently, comparison of biomechanical data is often limited or not permissible.

Many of the existing test protocols used to evaluate spinal fusion instrumentation involved application of a pure moment. Under this protocol, a moment is produced by applying two opposite forces on a pulley or bar system (27). In spine applications, a rotational moment of up to $10 \mathrm{Nm}$ is applied to the upper vertebrae where it is then transmitted equally throughout all spinal motion levels. However, physiologically, the human spine does not experience a constant bending moment. Thus, the pure moment protocol may not be the optimal testing methodology for analyzing disc replacement studies, where involvement at the adjacent segments is critical.

The hybrid testing protocol was developed recently for the sole purpose of addressing the adjacent level issue (6). It is more an extension of the pure moment protocol than an advancement in testing methodologies. In this alternative method, a pure moment is still applied in a similar procedure as described above, except that all spine conditions are rotated to a target end limit equal to that of the harvested spine condition. The rationale for this idea is that the patient will attempt to resume motion equal to that prior to surgery. The hybrid testing protocol has been advocated by Panjabi, Goel, et al as they believe it to be the most clinically relevant. However, the assumption that the preoperative range of motion is equal to the postoperative range of motion is one that does not have wide acceptance (28). In order for a fixation model to reach the same global rotation as the harvested condition, a higher moment input is required that typically exceeds the physiologic non-destructive load limit of $10 \mathrm{Nm}$. Whether a patient would, or even could, exert himself to reach this point remains suspect.

The protocol used in this study applied a compressive load to the specimen that induced a moment distribution across the spine. The maximum applied loads depended upon the specimen's structural capacity. A target moment of $8 \mathrm{Nm}$ was chosen for all of the specimens and stopped if the rotation exceeded a physiological limit of $30^{\circ}$. Accordingly, the analysis was carried out at the lowest common loading condition experience by any single spine condition. Chances of the specimen damage or nonphysiological loading were avoided by placing limits on the global stiffness and total range of motion. 


\section{Biomechanical Studies}

Several biomechanical studies have been put forth for Charite, ProDisc-L, and Maverick. Despite the use of either a pure moment or hybrid testing protocol by others there is a lack of uniformity in presentation. Studies include flexion and extension but not necessarily lateral bending and axial rotation. Fixation models are not always used either. Planar motions are always combined including flexion-extension. Table 5.1 summarizes operative level responses under the different testing parameters found in the literature.

Cunningham's group investigated the Charité at L4-L5 using a pure moment protocol (29). Cunningham found that Charité created a significantly higher axial rotation at the operative level compared to harvested. Flexion-extension and lateral bending were not significantly different. Fixation caused a significant decrease in motion under flexionextension and axial rotation.

O'Leary also uses a pure moment protocol to test the Charité at L5-S1 (30). Under flexion and extension, Charité was seen to create significantly higher rotation at both the operative and adjacent L4-L5 level.

As part of their study, Panjabi and Malcolmson used the Charité at L5-S1 and tested with the hybrid testing protocol (31). Charité did not cause any significant change from the harvested condition under flexion-extension. The fixation model caused a decrease in the operative level rotation in flexion-extension along with an increase at three adjacent levels (L1-L4). Axial rotation for both the Charite and fixation model was not significantly different than harvested.

To date, the only biomechanical study on ProDisc-L is by Panjabi et al. (32). The hybrid testing protocol was used to test the ProDisc-L at L5-S1 in a similar fashion as the Charité study discussed previously. ProDisc-L significantly increased the axial rotation and lateral bending at the operative level. Otherwise, no other significant changes were noted. Fixation caused a significant decrease at the operative level in flexion-extension and lateral bending but not axial rotation. Fixation also caused two adjacent levels to increase significantly in flexion-extension (T12-L1 and L3-L4). No adjacent level effects under fixation were noted in lateral bending and axial rotation. Given that Panjabi et al. used the same fixation method (pedicle screw and anterior plate) in both the Charite and ProDisc-L studies, the differences in adjacent level performance indicate the variability seen in cadavers.

Two studies have been published on the biomechanical characteristics of Maverick. Hitchon et al. inserted the Maverick at L4-L5 and applied a pure moment protocol (33). The disc replacement was show to cause a slight increase in motion in all directions. No significant differences were noted from harvested to disc replacement at any level.

Dmitriev et al. inserted the Maverick at L3-L4 and L4-L5 (34). The hybrid test method was used although the fixation conditions do not seem to match the same global 
Table 5.1: Biomechanical Studies and Results at the Operative Level.

\begin{tabular}{|c|c|c|c|c|c|c|c|c|}
\hline \multirow{3}{*}{$\begin{array}{l}\text { Author } \\
\text { Cunningham }\end{array}$} & \multirow{3}{*}{$\begin{array}{l}\text { Device } \\
\text { Charite }\end{array}$} & \multirow{3}{*}{$\begin{array}{l}\text { Location } \\
\text { L4-L5 }\end{array}$} & \multicolumn{6}{|c|}{ Significant Difference from Harvested } \\
\hline & & & \multicolumn{2}{|c|}{$\begin{array}{l}\text { Flexion- } \\
\text { Extension }\end{array}$} & \multicolumn{2}{|c|}{ Lateral } & \multicolumn{2}{|c|}{ Axial } \\
\hline & & & $\begin{array}{l}\text { Disc } \\
\mathrm{N}\end{array}$ & $\begin{array}{l}\text { Fix } \\
Y_{\text {(D) }}\end{array}$ & $\begin{array}{l}\text { Disc } \\
\mathrm{N}\end{array}$ & $\begin{array}{l}\text { Fix } \\
N\end{array}$ & $\begin{array}{l}\text { Disc } \\
Y_{(I)}\end{array}$ & $\begin{array}{l}\text { Fix } \\
Y_{\text {(D) }}\end{array}$ \\
\hline O’Leary & Charite & L5-S1 & $\mathrm{Y}_{(\mathrm{I})}$ & & & & & \\
\hline Panjabi & Charite & L5-S1 & $\mathrm{N}$ & $\mathrm{Y}_{(\mathrm{D})}$ & & & $\mathrm{N}$ & $\mathrm{N}$ \\
\hline Panjabi & ProDisc-L & L5-S1 & $\mathrm{N}$ & $Y_{(D)}$ & $\mathrm{Y}_{(\mathrm{I})}$ & Y & $\mathrm{Y}_{(\mathrm{I})}$ & $\mathrm{N}$ \\
\hline Panjabi & ProDisc-L & L4-S1 & $\mathrm{N}$ & $Y_{(D)}$ & $\mathrm{N}$ & $Y_{(D)}$ & $\mathrm{Y}_{(\mathrm{I})}$ & $\mathrm{Y}^{*}$ (D) \\
\hline Dmitriev & Maverick & L3-L5 & $Y_{(D)}$ & $\mathrm{Y}_{(\mathrm{D})}$ & $\mathrm{Y}_{(\mathrm{D})}$ & $\mathrm{Y}_{(\mathrm{D})}$ & $\mathrm{Y}_{(\mathrm{D})}$ & $\mathrm{Y}_{(\mathrm{D})}$ \\
\hline Hitchon & Maverick & L4-L5 & $\mathrm{N}$ & & $\mathrm{N}$ & & $\mathrm{N}$ & \\
\hline
\end{tabular}

Note: * L4-L5 only. Significant differences after a disc replacement or fixation model are indicated as yes $(\mathrm{Y})$ or no $(\mathrm{N})$. Significant increases (I) or decreases (D) are in parentheses. 
range of motion as required by the method. Under flexion-extension Maverick was found to significantly reduce motion compared to the harvested condition. Fixation further reduced the operative level rotations significantly from the Maverick condition without affecting adjacent levels. The same pattern of operative level motion reduction was seen in lateral bending and axial rotation. Unlike flexion-extension, lateral bending under fixation caused a significant increase in L2-L3 and L3-L4 adjacent levels. L2-L3 for axial and fixation was significantly higher.

Although the study by Dmitriev is not directly comparable to the current study, it is similar to Panjabi's ProDisc-L study where a second disc replacement was introduced at L4-L5 in addition to L5-S1. ProDisc-L did not significantly alter rotation at any levels under flexion-extension and lateral bending. Axial rotations at operative levels were significantly higher with the dual level ProDisc-L setup. Dual level fixation significantly affected all adjacent and operative levels in all directions except for L5-S1 in axial rotation.

The results gathered show the inconsistencies that arise in biomechanical studies. Researchers often have different testing parameters and objectives. Inconsistently altered adjacent levels from Panjabi's Charité and ProDisc-L studies indicate a high variance in cadaver performance. It then becomes impossible to differentiate the distinguishing features of each disc device from what is available in the literature.

\section{Finite Element Studies}

Finite element (FE) studies that evaluate the biomechanical stability of lumbar disc replacement devices provide complementary information to in vitro tissue tests. These studies provide additional information not attainable in a cadaver setting. Goel completed a finite element study on a lumbar spine model that had a Charité disc implanted at L5-S1 and simulated the hybrid testing protocol. The implanted condition caused a $19 \%$ increase in flexion and $44 \%$ increase in extension at the implanted L5-S1 level. Decreased rotation occurred at the immediate adjacent L4-L5 level of $12 \%$ in flexion and $28.6 \%$ in extension.

Rohlmann modeled the used the ProDisc-L at L3-L4(35). Loading was more complex than biomechanical studies as several muscle forces in addition to induced moments were applied to rotate the model. Rohlmann found the greatest differences ProDisc-L had from intact occurred at the operative level. ProDisc-L resulted in increases of $2.7^{\circ}$ in flexion, $7.1^{\circ}$ in extension, and $0.3^{\circ}$ axial compared to harvested. The study also determines the effect of anterior-posterior positioning of the disc. Flexion was the most affected by positioning. Starting at a posterior position, a $4 \mathrm{~mm}$ shift anterior resulted in an decrease of $4^{\circ}$ rotation in flexion.

Dooris modeled the L3-L4 level and inserted a posterior positioned disc implant similar to Maverick. A pure moment was used to induce loading. Shifting of the anterior- 
posterior position by $8 \mathrm{~mm}$ resulted in a decrease of $1.8^{\circ}$ when further anterior. The results coincide with those of Rohlmanan although the results are not as dramatic.

\section{Clinical Studies}

Only two groups have published clinical findings that directly compare different disc prostheses. The first group study used the Charité and ProDisc-L with a minimum 3 year follow up (36). A significant difference was seen in L5-S1 patients: Charité patients had a higher range of motion than ProDisc-L. Degeneration of the facets at operative level and adjacent discs occurred in a significant number of both groups. However, no significant differences concerning degeneration at the adjacent levels or facets were found between the two discs populations.

In the second study, the Charité, ProDisc-L, and Maverick disc prostheses were compared (37). Subjects were not randomized: a different surgeon screened and operated for each disc prosthesis. The discs were implanted at either the L4-L5 or L5-S1 level. The disc performances under flexion-extension were indistinguishable and, on average, only improved that range of motion by $2^{\circ}$.

Longer termed clinical studies are still ongoing. Huang's patients averaged 8.7 years of implantation with ProDisc-L. Only $66 \%$ of patients had flexion-extension motion of at least $2^{\circ}$. Furthermore, $24 \%$ of patients were determined to have radiographic adjacent level degeneration while none required further surgical procedure.

\section{Flexibility Discussion}

Flexibility results in this study are typical for any disc prosthesis study. Flexibility decreases under flexion for all instrumented conditions while increasing in extension. There are likely two major factors explaining this occurrence. The first is that insertion of an artificial disc requires the near complete loss of the anterior longitudinal ligament functionality. Secondly, during the discectomy the cadaver assumes a new neutral position. This change is more lenient for disc replacement but not attempting realignment during fixation results in a starting position slightly extended.

The surgical alterations to sagittal balance and positioning did not exert as much influence on lateral bending and axial rotation. Surgery preserved operative level disc annulus as much as possible thereby preserving much of lateral bending and axial rotation. In Mathews' review of the Maverick he noted a loss in stiffness under lateral bending whereas flexion-extension and axial rotation were slightly stiffer (38). In this study, all Mavericks were tested after ProDisc-L and thus Maverick specimens were more susceptible to the wear effects of cadaver testing. Despite this issue, only in flexion did Maverick deviate from the flexibility performance of ProDisc-L. Maverick was significantly more flexible in flexion than ProDisc-L. 


\section{MSU Percent Change in Contribution}

ProDisc-L and Maverick do not appear to alter the percent change in contribution profiles from harvested. Adjacent level changes in fixation were noted by combining flexion-extension. Combining the results for flexion and extension is not ideal due to the different structures that are loaded in both directions. However, this was the only way in which a significant difference at an adjacent level (L3-L4) appeared. The practice of combined flexion-extension was previously mentioned in the biomechanical studies section. It is possible that those researchers encountered the same situation.

The only significant difference between Prodisc-L or Maverick from a control was under right lateral bending. Maverick specimen rotations were significantly higher at the operative level than fixation. This is due to a combination of unbalanced specimen physiology and surgeon error in alignment. The issues are resolved by combining right and left lateral. The left and right axial percent change in contribution profiles are coupled more closely than lateral. Both left and right axial rotation for fixation indicated a decrease in operative level rotation while observing the same significant at L3-L4. Lateral and axial results are in contrast to Panjabi's ProDisc-L paper stating lateral bending and axial rotation were significantly higher at the operative level as a result of disc replacement.

\section{Adjacent Level Effects}

There is no consensus as to why adjacent level degeneration occurs in fusion patients. The conventional thought is that a significant increase at a non-operative level gives rise to the phenomena of adjacent level degeneration. Those workers who do not subscribe to the existence of adjacent level degeneration believe that once degenerative disc disease is present, all of the patient's intervertebral discs are susceptible. Patients are genetically predisposed to weak disc. If this is true no instrumentation would be able to prevent the phenomenon of adjacent level degeneration. Lehmann, Luk, and Penta (14) find no evidence of adjacent segment degeneration in long term (10+ years) patients. Groups that support adjacent effects include Rahm (39) and Whitecloud (40). A study published in 2004 by Ghiselli looked at 223 patients after an average of 6.7 years for and found that patients with multiple level fusions were less likely than single level patients to develop symptomatic adjacent levels (41). Their reasoning was that degeneration had an equal probability of occurrence. Multi-level fusion simply reduced the chance of a segment degenerating.

Biomechanical studies often hint at adjacent level degeneration due to fusion despite the lack of conclusiveness. It is usually taken for granted that motion preservation is in the best interest of the patient. Less thought is given to the possibility that keeping a disc joint mobile may contribute to adjacent degeneration as well. When longer term studies of disc arthroplasty are completed, more insight will be gained from the origins of adjacent level degeneration. 
In this study, only until flexion and extension is combined does an adjacent level (L2-L3) show significant differentiation from the harvested under fixation. No significant differences at the adjacent levels were notes when separating flexion and extension. Although no literature has been critical of this practice it is obvious there is a loss in detail that is important when incorporated into a disc replacement study.

There is a strong indication that both ProDisc-L and Maverick will preserve motion at all levels. Pedicle screw fixation however, does not retain the harvested state's motion at all of the adjacent levels. L2-L3 in flexion-extension and L3-L4 in axial rotation both rotate higher after fixation. Specimen variability is uncontrolled due to limited resources and its effects were noted previously under the biomechanical studies section. Although no clinical fusion studies have focused on the effects at specific levels, Siepe et al have with a total disc replacement. Siepe's clinical study reports higher satisfaction with L4-L5 disc replacement patients than those with L5-S1. Motion preservation at L5-S1 was not as beneficial as it was at L4-L5. Adjacent level effects may still be a concern but may be less of a concern for fusions at L5-S1 thus explaining the low indications of adjacent level changes.

\section{Design Factor Influences}

Two major distinguishing features of ProDisc-L and Maverick are discussed. Design factor discussion in literature is mainly on the influence of flexion and extension and is thus presented as such. The first feature is the anterior-posterior depth positions the disc replacements assume after implantation.

Prodisc-L and Maverick discs are both $27 \mathrm{~mm}$ in depth but the Maverick's ball and socket sits farther posterior on the device itself. As explained in the Chapter 3 , flouroimaging was used to determine the distances from ball center to the posterior longitudinal ligament. There was a difference of nearly $5 \mathrm{~mm}$ between the disc centers, Maverick obviously being closer to the ligament. Dooris and Rolhmann used finite element models and postulated that the further posterior the ball and socket center was located, the higher the operative level rotation would be. Dooris used an anteriorposterior depth differential of $8 \mathrm{~mm}$ in his analysis along with a $6 \mathrm{Nm}$ load. Based upon the results in this study, ProDisc-L rotated less than Maverick in extension instead of flexion. However, since the combined flexion and extension was used in the defense of fixation's affect on adjacent level changes, it is consistent to include combined flexion and extension in the disc replacement discussion. When combining Dooris's results, flexion-extension decreases more than $15 \%$ when moving $8 \mathrm{~mm}$ further anterior. In the current study, the decrease in percent change in contribution is $21 \%$ albeit with a high standard deviation of $16 \%$.

The findings here indicate that finite element modeling claims are correct. However, as mentioned in the adjacent level discussion, the details of flexion and extension are lost. The disconnection between biomechanical studies and finite element studies may be prevalent elsewhere as well. Mathews' testing of the Maverick mentions 
no significant differences in range of motion measurements expect in extension (as opposed to the Dooris and Rolhmann claim of flexion) from the harvested state (38). The author does not give further detail on this finding from a private company cadaver test.

The second feature of interest is the disc replacement radius of curvature. The ball and socket of the disc replacements are similarly sized. It has been proposed that the radius of curvature of the disc replacement affects the amount of translation (shear) (7). A disc replacement with a large radius of curvature would allow for posterior translation in extension that may overstress the facet joints and ligaments. ProDisc-L has a slightly larger radius of curvature but the result is less than $1 \mathrm{~mm}$ difference from the Maverick. The design of ProDisc-L and Maverick cause the center of rotation to reside slightly below the inferior endplate while the natural L5-S1 center of rotation has been estimated to lie within the disc space. Since ProDisc-L and Maverick are very similar, curvature should not be a major factor in differentiation. The results obtained in this study were insufficient in identifying any radius of curvature effect. However, in other disc devices this may play a role as a distinguishing feature.

\section{Limitations}

It is unrealistic to assume symptomatic patients to move similarly to healthy ones. The response of a healthy cadaver to a disc replacement is likely to create a wide range of results. This is partially seen when observing the percent change in contribution at the operative levels. Although the averages are such that percent change in contribution is similar at the operative level, the standard deviations are usually the highest at that level. Use of asymptomatic cadavers limits the purpose of disc replacement which is to treat degenerative disc disease.

Although replication of in vivo motion is a goal it has not been met by any biomechanical lab. Cadaver testing cannot simulate the postoperative recuperation time afforded to in vivo patients. Based upon the various testing protocols still in use, it is clear there is not a consensus on a universal testing system. As seen in the discussion of other lab results, inconsistencies abound even when using the same general testing protocol. All testing protocols can be improved upon as none attempt to replicate the true complexities of muscle forces.

For this study, one question is the validity of the current protocol in its suitability for lumbar cadavers. The only alteration from cervical to lumbar is an $8 \mathrm{Nm}$ loading limit with an additional $30^{\circ}$ global rotation end limit. Lumbar physiology and symptoms of lumbar pain are sufficiently different from that of the cervical. Questions may arise as to what effect if any using essentially the same protocol has in data bias.

The sensitivity of the testing protocol is likely not high enough. While sufficient to determine differentiation compared to fixation , ProDisc-L and Maverick follow essentially the same mechanical design rationale: constrained ball and socket design. In a testing protocol designed to observe all five motion segments, it is possible that motion 
compensation for the operative level is redistributed in such a manner that it becomes difficult to detect. Short of lengthy endurance testing, no insight towards stresses and strain can be deduced from the protocol.

Using the same specimens throughout the testing and hence enabling repeated measures decreases variation due to the difference specimens. The assumption that the first disc replacement implant and removal did not cause structural alterations cannot be made. In clinical studies, disc replacement removal has been followed with some type of fusion never another disc replacement. In a cadaver setting, the effects may explain the relative increase in flexibility seen in Maverick when compared to ProDisc-L. It is unheard of in clinical setting to remove a disc replacement and reinserting another. In the cadaver setting, removal of a disc can cause permanent deformation to spinal structure and create a variable that is difficult to account for in a comparative disc study such as this one. To identify such a potential problem, after the specimens were fitted and tested with ProDisc-L and then Maverick, ProDisc-L was reinserted and tested a second time.

ProDisc-L, Maverick and other artificial discs are approved for implantation throughout the lumbar spine. The analysis here is at the lowest segment with no inferior level. It is possible that different implantation levels yield different motion redistribution at inferior levels. Clinical researchers believe patient satisfaction is related to the afflicted level (42). This limits the currents study's application to only the L5-S1 level.

All biomechanical studies have a time frame in which cadaver testing is effective. The longer a study is the more time the specimens may experience permanent relaxation and degradation. This study was relatively short with only three surgical conditions including a pedicle fixation that could be engaged or disengaged without affecting the disc replacement systems. Fortunately, single level disc replacement is usually the first procedure performed thus when comparing results to other labs the question of specimen quality does not arise. 


\section{CHAPTER 6: CONCLUSIONS}

With Maverick disc prosthesis set to enter the US market in the very near future, it will likely become the primary competitor to ProDisc-L disc implant. Several factors will likely influce the outcome of which device is preferred over the other that include ease of insertion, cost, and most importantly patient satisfaction. Short term indications are that disc replacement reduce the recovery time relative to fusion surgery, which also directly reduces overall cost (43). However, the need for fusion surgery will continue, but some clinical situations exists that may result in disc arthroplasty being a better alternative to fusion surgery; for example, preventing or controlling adjacent segement disc and treating early stage disc degeneration. In addition to disc arthroplasty surgery, advancements in other non-fusion surgical procedures are being developed, such as, dynamic stabilization and nucleus arthroplasty.

Implantation of the ProDisc-L or Maverick disc prosthesis did not significantly alter the spinal motion of the multi-level lumbar spine. In contrast, pedicle screw fixation not only caused a decrease in operative level rotation, but it also increased the compensatory rotation that occurred at the adjacent, non-operative spinal levels under flexion-extension and axial rotation. Long term clinical studies comparing the outcome of fusion and disc replacement surgeries are only now emerging and will help determine the success of this alterative procedure.

Differences in ball and socket positioning for the ProDisc-L and Maverick after implantation affected rotational allowance according to findings from finite element studies reported by other researchers. This is in contrast to the present study, where no significant differences were detected. Results such as those of ProDisc-L are also different from that found in the literature, highlighting the influence of testing protocol on results. In order to further differentiate devices such as ProDisc-L and Maverick, better knowledge of what and how structures are affected is necessary. 


\section{CHAPTER 7: RECOMMENDATIONS FOR FUTURE STUDY}

This study compared two similar disc prostheses that were of a ball and socket design and used a central keel for primary fixation to the vertebral endplates. It would be of interest to compare these results to other disc prostheses, such as semi-constrained Charité disc. Biomechanical data from such a study would further compliment any clinical findings that show differences between the different prosthetic disc designs.

The radius of curvature as a design factor was mentioned. Its ideal effects were proposed to be related mainly to force and shear data. With a difference of less than 1 $\mathrm{mm}$ and no results that could be accounted for by curvature, it was assumed to be insignificant towards the discussion. However other sensors such as the facet sensor used previously may be able to distinguish differences. Abnormal forces and wear are also applicable to in vivo.

Lastly, disc prostheses' are designed to maintain or restore the complex motion of the spine. In this study a sequence of two-dimensional tests were performed within sected anatomical planes of motion (i.e., flexion/extension, lateral bending, and axial rotation). The next generation of testing protocols should give some consideration to the coupled motion conditions of the spine. This could be achieved by using more advanced testing systems like the Spine Robot developed in our Biomechanics Lab. In the end, the outcome from such an advanced testing protocol could be compared back to the traditional planar studies to highlight any similarities or difference that exist between the two methodologies. 


\section{LIST OF REFERENCES}

1. Luoma K, Riihimaki H, Luukkonen R, Raininko R, Viikari-Juntura E, Lamminen A. Low back pain in relation to lumbar disc degeneration. Spine. 2000 Feb $15 ; 25(4): 487-92$.

2. Boden SD, Balderston RA, Heller JG, Hanley EN, Jr., Zigler JE. An AOA critical issue. Disc replacements: this time will we really cure low-back and neck pain? J Bone Joint Surg Am. 2004 Feb;86-A(2):411-22.

3. Bono CM, Garfin SR. History and evolution of disc replacement. Spine J. 2004 Nov-Dec;4(6 Suppl):145S-50S.

4. Errico TJ. Lumbar disc arthroplasty. Clin Orthop Relat Res. 2005 Jun(435):10617.

5. Lee CK, Goel VK. Artificial disc prosthesis: design concepts and criteria. Spine J. 2004 Nov-Dec;4(6 Suppl):209S-18S.

6. Panjabi MM. Hybrid multidirectional test method to evaluate spinal adjacent-level effects. Clin Biomech (Bristol, Avon). 2007 Mar;22(3):257-65.

7. Huang RC, Wright TM, Panjabi MM, Lipman JD. Biomechanics of nonfusion implants. Orthop Clin North Am. 2005 Jul;36(3):271-80.

8. Eubanks JD, Lee MJ, Cassinelli E, Ahn NU. Does lumbar facet arthrosis precede disc degeneration? A postmortem study. Clin Orthop Relat Res. 2007 Nov;464:184-9.

9. Butler D, Trafimow JH, Andersson GB, McNeill TW, Huckman MS. Discs degenerate before facets. Spine. 1990 Feb;15(2):111-3.

10. Fujiwara A, Tamai K, Yamato M, An HS, Yoshida H, Saotome K, et al. The relationship between facet joint osteoarthritis and disc degeneration of the lumbar spine: an MRI study. Eur Spine J. 1999;8(5):396-401.

11. Wong DA, Annesser B, Birney T, Lamond R, Kumar A, Johnson S, et al. Incidence of contraindications to total disc arthroplasty: a retrospective review of 100 consecutive fusion patients with a specific analysis of facet arthrosis. Spine J. 2007 Jan-Feb;7(1):5-11.

12. Pintar FA, Yoganandan N, Myers T, Elhagediab A, Sances A, Jr. Biomechanical properties of human lumbar spine ligaments. J Biomech. 1992 Nov;25(11):13516. 
13. Fritzell P, Hagg O, Wessberg P, Nordwall A. Chronic low back pain and fusion: a comparison of three surgical techniques: a prospective multicenter randomized study from the Swedish lumbar spine study group. Spine. 2002 Jun 1;27(11):1131-41.

14. Lehmann TR, Spratt KF, Tozzi JE, Weinstein JN, Reinarz SJ, el-Khoury GY, et al. Long-term follow-up of lower lumbar fusion patients. Spine. 1987 Mar;12(2):97-104.

15. Geisler FH, Guyer RD, Blumenthal SL, McAfee PC, Cappuccino A, Bitan F, et al. Patient selection for lumbar arthroplasty and arthrodesis: the effect of revision surgery in a controlled, multicenter, randomized study. J Neurosurg Spine. 2008 Jan;8(1):13-6.

16. Ozgur BM, Aryan HE, Pimenta L, Taylor WR. Extreme Lateral Interbody Fusion (XLIF): a novel surgical technique for anterior lumbar interbody fusion. Spine J. 2006 Jul-Aug;6(4):435-43.

17. Marotta N, Cosar M, Pimenta L, Khoo LT. A novel minimally invasive presacral approach and instrumentation technique for anterior L5-S1 intervertebral discectomy and fusion: technical description and case presentations. Neurosurg Focus. 2006;20(1):E9.

18. Li C, Xu HZ, Wang XY, Ni WF, Chi YL, Huang QS, et al. [Comparison of the paraspinal muscle change of percutaneous and open pedicle screw fixation in the treatment for thoracolumbar fractures]. Zhonghua Wai Ke Za Zhi. 2007 Jul 15;45(14):972-5.

19. Zigler JE, Burd TA, Vialle EN, Sachs BL, Rashbaum RF, Ohnmeiss DD. Lumbar spine arthroplasty: early results using the ProDisc II: a prospective randomized trial of arthroplasty versus fusion. J Spinal Disord Tech. 2003 Aug;16(4):352-61.

20. Geisler FH, Blumenthal SL, Guyer RD, McAfee PC, Regan JJ, Johnson JP, et al. Neurological complications of lumbar artificial disc replacement and comparison of clinical results with those related to lumbar arthrodesis in the literature: results of a multicenter, prospective, randomized investigational device exemption study of Charite intervertebral disc. Invited submission from the Joint Section Meeting on Disorders of the Spine and Peripheral Nerves, March 2004. J Neurosurg Spine. 2004 Sep;1(2):143-54.

21. DiAngelo DJ, Foley KT. An improved biomechanical testing protocol for evaluating spinal arthroplasty and motion preservation devices in a multilevel human cadaveric cervical model. Neurosurg Focus. 2004 Sep 15;17(3):E4. 
22. DiAngelo DJ, Foley KT, Morrow BR, Schwab JS, Song J, German JW, et al. In vitro biomechanics of cervical disc arthroplasty with the ProDisc-C total disc implant. Neurosurg Focus. 2004 Sep 15;17(3):E7.

23. Schwab JS, Diangelo DJ, Foley KT. Motion compensation associated with singlelevel cervical fusion: where does the lost motion go? Spine. 2006 Oct $1 ; 31(21): 2439-48$.

24. Kim DH, Cammisa Jr. FP, Fessler RG. Dynamic Reconstruction of the Spine. New York: Thieme Medical Publishers, Inc.; 2006.

25. DiAngelo DJ, Kitchel S, McVay BJ, Scifert JL, Cornwall GB. Bioabsorbable anterior lumbar plate fixation in conjunction with anterior interbody fusion cages. Orthopedics. 2002 Oct;25(10 Suppl):s1157-65; discussion s65.

26. DiAngelo DJ, Scifert JL, Kitchel S, Cornwall GB, McVay BJ. Bioabsorbable anterior lumbar plate fixation in conjunction with cage-assisted anterior interbody fusion. J Neurosurg. 2002 Nov;97(4 Suppl):447-55.

27. Goel VK, Panjabi MM, Patwardhan AG, Dooris AP, Serhan H. Test protocols for evaluation of spinal implants. J Bone Joint Surg Am. 2006 Apr;88 (2 Suppl ):1039.

28. Chou WY, Hsu CJ, Chang WN, Wong CY. Adjacent segment degeneration after lumbar spinal posterolateral fusion with instrumentation in elderly patients. Arch Orthop Trauma Surg. 2002 Feb;122(1):39-43.

29. Cunningham BW. Basic scientific considerations in total disc arthroplasty. Spine J. 2004 Nov-Dec;4(6 Suppl):219S-30S.

30. O'Leary P, Nicolakis M, Lorenz MA, Voronov LI, Zindrick MR, Ghanayem A, et al. Response of Charite total disc replacement under physiologic loads: prosthesis component motion patterns. Spine J. 2005 Nov-Dec;5(6):590-9.

31. Panjabi M, Malcolmson G, Teng E, Tominaga Y, Henderson G, Serhan H. Hybrid testing of lumbar Charite discs versus fusions. Spine. 2007 Apr 20;32(9):959-66; discussion 67.

32. Panjabi M, Henderson G, Abjornson C, Yue J. Multidirectional testing of oneand two-level ProDisc-L versus simulated fusions. Spine. 2007 May 20;32(12):1311-9.

33. Hitchon PW, Eichholz K, Barry C, Rubenbauer P, Ingalhalikar A, Nakamura S, et al. Biomechanical studies of an artificial disc implant in the human cadaveric spine. J Neurosurg Spine. 2005 Mar;2(3):339-43. 
34. Dmitriev AE, Gill NW, Kuklo TR, Rosner MK. Effect of multilevel lumbar disc arthroplasty on the operative- and adjacent-level kinematics and intradiscal pressures: an in vitro human cadaveric assessment. Spine J. 2008 NovDec;8(6):918-25

35. Rohlmann A, Zander T, Bergmann G. Effect of total disc replacement with ProDisc on intersegmental rotation of the lumbar spine. Spine. $2005 \mathrm{Apr}$ $1 ; 30(7): 738-43$.

36. Shim CS, Lee SH, Shin HD, Kang HS, Choi WC, Jung B, et al. Charite versus ProDisc: a comparative study of a minimum 3-year follow-up. Spine. 2007 Apr 20;32(9):1012-8.

37. Tournier C, Aunoble S, Le Huec JC, Lemaire JP, Tropiano P, Lafage V, et al. Total disc arthroplasty: consequences for sagittal balance and lumbar spine movement. Eur Spine J. 2007 Mar;16(3):411-21.

38. Mathews HH, Lehuec JC, Friesem T, Zdeblick T, Eisermann L. Design rationale and biomechanics of Maverick Total Disc arthroplasty with early clinical results. Spine J. 2004 Nov-Dec;4(6 Suppl):268S-75S.

39. Rahm MD, Hall BB. Adjacent-segment degeneration after lumbar fusion with instrumentation: a retrospective study. J Spinal Disord. 1996 Oct;9(5):392-400.

40. Whitecloud TS, 3rd, Davis JM, Olive PM. Operative treatment of the degenerated segment adjacent to a lumbar fusion. Spine. 1994 Mar 1;19(5):531-6.

41. Ghiselli G, Wang JC, Hsu WK, Dawson EG. L5-S1 segment survivorship and clinical outcome analysis after L4-L5 isolated fusion. Spine. 2003 Jun 15;28(12):1275-80; discussion 80.

42. Siepe CJ, Mayer HM, Heinz-Leisenheimer M, Korge A. Total lumbar disc replacement: different results for different levels. Spine. 2007 Apr 1;32(7):78290.

43. Levin DA, Bendo JA, Quirno M, Errico T, Goldstein J, Spivak J. Comparative charge analysis of one- and two-level lumbar total disc arthroplasty versus circumferential lumbar fusion. Spine. 2007 Dec 1;32(25):2905-9. 


\section{APPENDICES}


Appendix A:

MSU Rotation 

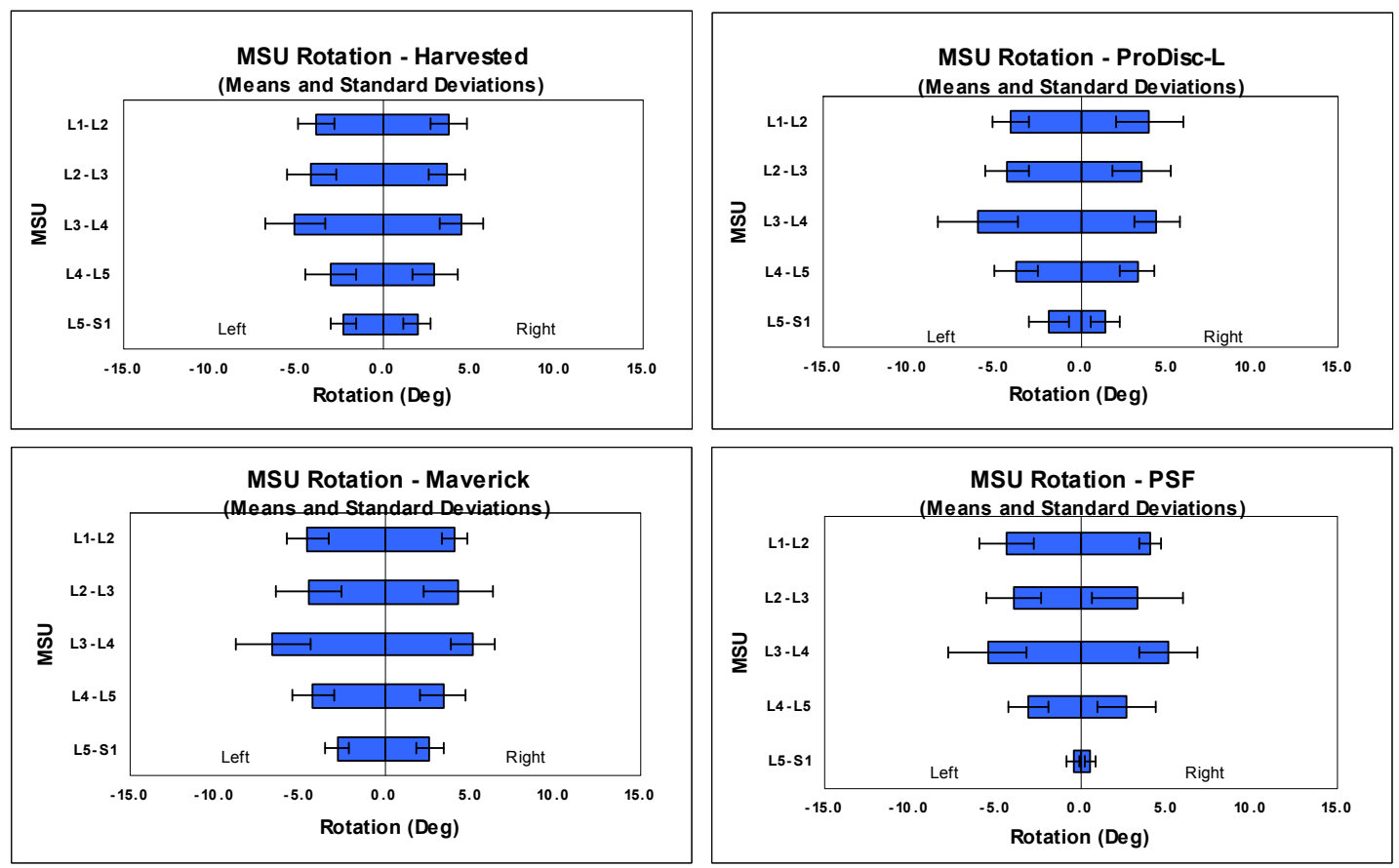

Figure A.1: MSU Rotations of the Different Spine Conditions for Left and Right Lateral. 

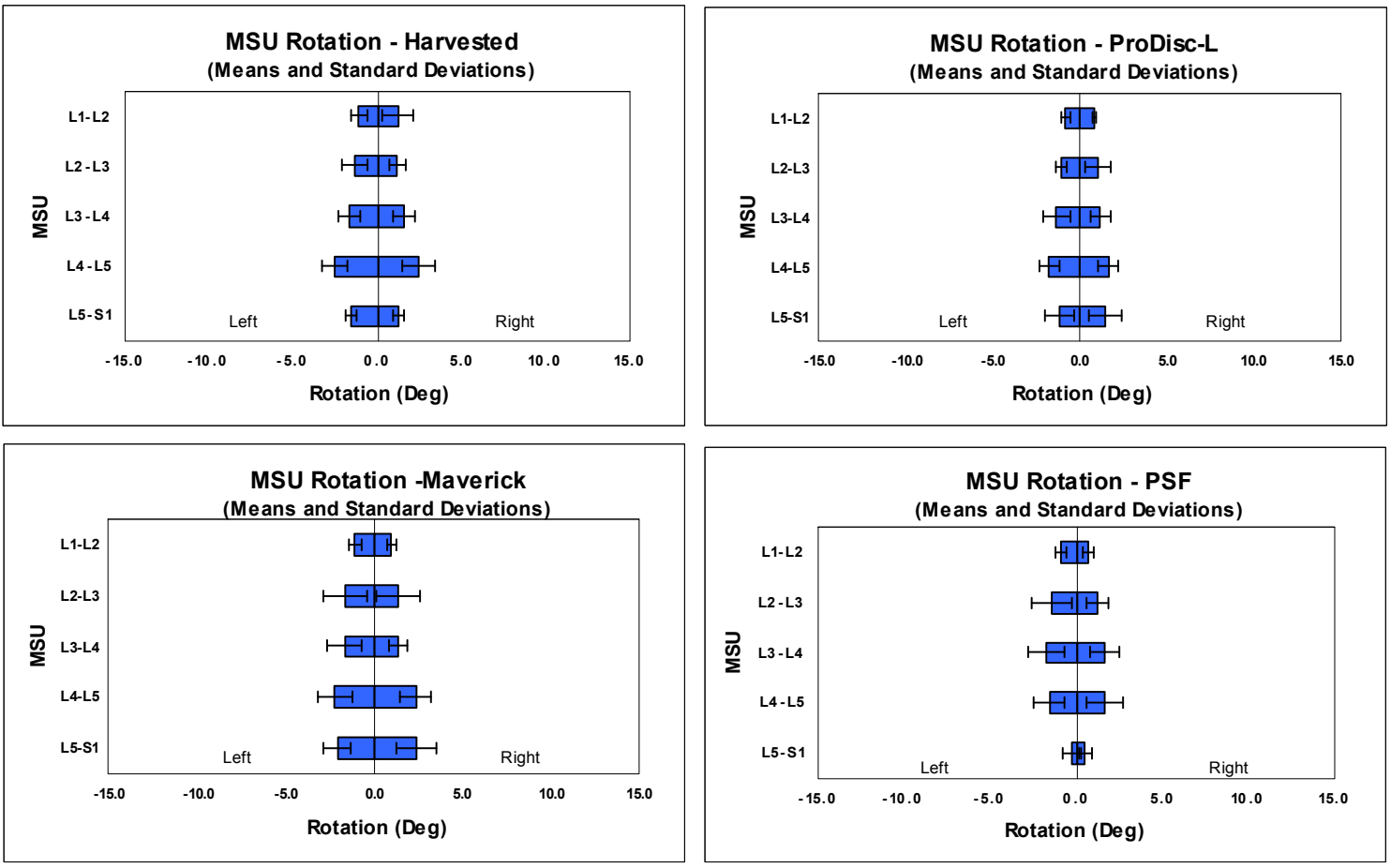

Figure A.2: MSU Rotations of the Different Spine Conditions for Left and Right Axial. 
Table A.1: MSU Rotation ( $\left.{ }^{\circ}\right)$ Mean and Standard Deviation.

\begin{tabular}{|c|c|c|c|c|}
\hline \multirow[b]{2}{*}{ Direction } & \multicolumn{4}{|c|}{ Construct } \\
\hline & Harvested & ProDisc-L & Maverick & Pedicle Screw \\
\hline \multicolumn{5}{|l|}{ Flexion } \\
\hline L1-L2 & $5.3 \pm 2.5$ & $3.1 \pm 1.9$ & $4.7 \pm 2.1$ & $4.3 \pm 2.7$ \\
\hline L2-L3 & $4.3 \pm 1.6$ & $3.0 \pm 1.1$ & $3.9 \pm 1.7$ & $2.9 \pm 1.0$ \\
\hline L3-L4 & $5.6 \pm 1.9$ & $3.3 \pm 1.2$ & $5.0 \pm 1.8$ & $3.9 \pm 2.1$ \\
\hline L4-L5 & $5.1 \pm 1.6$ & $3.6 \pm 1.1$ & $4.4 \pm 1.7$ & $3.6 \pm 1.7$ \\
\hline L5-S1 & $5.5 \pm 3.0$ & $3.3 \pm 1.9$ & $5.2 \pm 2.5$ & $0.8 \pm 0.6$ \\
\hline \multicolumn{5}{|l|}{ Extension } \\
\hline L1-L2 & $2.6 \pm 1.3$ & $4.7 \pm 1.4$ & $4.8 \pm 1.4$ & $4.0 \pm 1.9$ \\
\hline L2-L3 & $1.8 \pm 1.1$ & $4.0 \pm 1.3$ & $3.7 \pm 1.7$ & $3.4 \pm 2.2$ \\
\hline L3-L4 & $2.4 \pm 0.9$ & $4.3 \pm 1.5$ & $4.2 \pm 1.3$ & $3.9 \pm 1.8$ \\
\hline L4-L5 & $2.5 \pm 1.7$ & $5.3 \pm 2.5$ & $4.0 \pm 2.0$ & $4.1 \pm 2.9$ \\
\hline L5-S1 & $3.4 \pm 1.8$ & $5.6 \pm 2.7$ & $7.4 \pm 3.9$ & $0.6 \pm 0.1$ \\
\hline \multicolumn{5}{|l|}{ Left Lateral } \\
\hline L1-L2 & $3.9 \pm 0.9$ & $4.0 \pm 1.0$ & $4.5 \pm 1.2$ & $4.3 \pm 1.5$ \\
\hline L2-L3 & $3.9 \pm 1.4$ & $4.2 \pm 1.2$ & $4.5 \pm 1.9$ & $3.9 \pm 1.6$ \\
\hline L3-L4 & $5.6 \pm 2.0$ & $6.0 \pm 2.3$ & $6.6 \pm 2.2$ & $5.4 \pm 2.3$ \\
\hline L4-L5 & $3.0 \pm 1.3$ & $3.7 \pm 1.2$ & $4.2 \pm 1.1$ & $3.0 \pm 1.1$ \\
\hline L5-S1 & $2.2 \pm 0.6$ & $1.8 \pm 1.2$ & $2.8 \pm 0.7$ & $0.4 \pm 0.3$ \\
\hline \multicolumn{5}{|l|}{ Right Lateral } \\
\hline L1-L2 & $3.7 \pm 0.9$ & $4.0 \pm 1.9$ & $4.0 \pm 0.7$ & $4.0 \pm 0.6$ \\
\hline L2-L3 & $3.4 \pm 1.1$ & $3.5 \pm 1.7$ & $4.2 \pm 2.0$ & $3.2 \pm 2.6$ \\
\hline L3-L4 & $4.6 \pm 1.1$ & $4.4 \pm 1.3$ & $5.1 \pm 1.2$ & $5.1 \pm 1.7$ \\
\hline L4-L5 & $2.8 \pm 1.2$ & $3.3 \pm 1.0$ & $3.4 \pm 1.3$ & $2.6 \pm 1.6$ \\
\hline L5-S1 & $1.8 \pm 0.7$ & $1.4 \pm 0.8$ & $2.6 \pm 0.7$ & $0.5 \pm 0.3$ \\
\hline \multicolumn{5}{|l|}{ Left Axial } \\
\hline L1-L2 & $0.9 \pm 0.6$ & $0.8 \pm 0.2$ & $1.0 \pm 0.3$ & $0.9 \pm 0.3$ \\
\hline L2-L3 & $1.2 \pm 0.7$ & $1.0 \pm 0.3$ & $1.5 \pm 1.2$ & $1.4 \pm 1.1$ \\
\hline L3-L4 & $1.5 \pm 0.6$ & $1.3 \pm 0.7$ & $1.6 \pm 0.9$ & $1.7 \pm 1.0$ \\
\hline L4-L5 & $2.3 \pm 0.9$ & $1.7 \pm 0.5$ & $2.1 \pm 0.9$ & $1.5 \pm 0.8$ \\
\hline L5-S1 & $1.5 \pm 0.3$ & $1.1 \pm 0.8$ & $2.0 \pm 0.7$ & $0.2 \pm 0.5$ \\
\hline \multicolumn{5}{|l|}{ Right Axial } \\
\hline L1-L2 & $1.0 \pm 0.8$ & $0.8 \pm 0.1$ & $1.0 \pm 0.2$ & $0.7 \pm 0.3$ \\
\hline L2-L3 & $1.1 \pm 0.4$ & $1.0 \pm 0.7$ & $1.4 \pm 1.2$ & $1.2 \pm 0.6$ \\
\hline L3-L4 & $1.4 \pm 0.7$ & $1.2 \pm 0.5$ & $1.3 \pm 0.5$ & $1.6 \pm 0.8$ \\
\hline L4-L5 & $2.2 \pm 1.0$ & $1.6 \pm 0.5$ & $2.3 \pm 0.8$ & $1.6 \pm 1.0$ \\
\hline L5-S1 & $1.2 \pm 0.3$ & $1.4 \pm 0.9$ & $2.3 \pm 1.1$ & $0.5 \pm 0.4$ \\
\hline
\end{tabular}


Appendix B:

Normalized Flexibility 
Table B.1: Normalized Flexibility Statistics (ANOVA + SNK).

\begin{tabular}{lll}
\hline Direction and Construct & P-value $(\mathrm{p})$ & $\begin{array}{l}\text { Significant } \\
\text { Difference } \\
(\mathrm{p}<0.05)\end{array}$ \\
\hline Flexion & 0.002 & Yes \\
H vs PD & 0.007 & Yes \\
H vs M & - & No \\
H vs PSF & 0.003 & Yes \\
PD vs M & 0.047 & Yes \\
PD vs PSF & - & No \\
M vs PSF & 0.031 & Yes \\
Extension & 0.008 & Yes \\
H vs PD & 0.013 & Yes \\
H vs M & 0.008 & Yes \\
H vs PSF & - & No \\
PD vs M & - & No \\
PD vs PSF & - & No \\
M vs PSF & - & No \\
Left Lateral & & No \\
Right Lateral & 0.079 & No \\
Left Axial & 0.072 & No \\
Right Lateral & 0.088 & No \\
\hline
\end{tabular}

H: Harvested

PD: ProDisc-L

M: Maverick

PSF: Pedicle Screw Fixation 
Appendix C:

MSU Percent Change in Contribution 
Table C.1: MSU Percent Change in Contribution Statistics (ANOVA + SNK).

\begin{tabular}{|c|c|c|c|c|c|c|c|c|c|c|}
\hline \multirow{3}{*}{$\begin{array}{c}\text { Direction and } \\
\text { Construct }\end{array}$} & \multicolumn{10}{|c|}{ Motion Segment Unit } \\
\hline & \multicolumn{2}{|c|}{ L1-L2 } & \multicolumn{2}{|c|}{ L2-L3 } & \multicolumn{2}{|c|}{ L3-L4 } & \multicolumn{2}{|c|}{ L4-L5 } & \multicolumn{2}{|c|}{ L5-S1 } \\
\hline & $\mathrm{p}$ & SD & $\mathrm{p}$ & SD & $\mathrm{p}$ & SD & $\mathrm{p}$ & SD & $\mathrm{p}$ & SD \\
\hline $\begin{array}{l}\text { Flexion } \\
\mathrm{H} \text { vs Pd } \\
\mathrm{H} \text { vs M }\end{array}$ & 0.255 & No & 0.276 & No & 0.249 & No & 0.437 & No & 0.036 & Yes \\
\hline $\mathrm{H}$ vs PSF & & & & & & & & & 0.05 & Yes \\
\hline PD vs M & & & & & & & & & 0.04 & Yes \\
\hline PD vs PSF & & & & & & & & & 0.036 & Yes \\
\hline M vs PSF & & & & & & & & & & \\
\hline $\begin{array}{c}\text { Extension } \\
\mathrm{H} \text { vs } \mathrm{Pd} \\
\mathrm{H} \text { vs } \mathrm{M}\end{array}$ & 0.425 & No & 0.112 & No & 0.044 & No & 0.07 & No & 0.001 & Yes \\
\hline $\mathrm{H}$ vs PSF & & & & & & & & & 0.001 & Yes \\
\hline PD vs M & & & & & & & & & & \\
\hline PD vs PSF & & & & & & & & & 0.001 & Yes \\
\hline M vs PSF & & & & & & & & & 0.001 & Yes \\
\hline $\begin{array}{c}\text { Flx }+ \text { Ext } \\
\text { H vs Pd }\end{array}$ & 0.116 & No & 0.01 & Yes & 0.044 & No & 0.194 & No & 0.001 & Yes \\
\hline H vs M & & & & & & & & & & \\
\hline $\begin{array}{l}\mathrm{H} \text { vs } \mathrm{PSF} \\
\mathrm{PD} \text { vs } \mathrm{M}\end{array}$ & & & 0.009 & Yes & & & & & 0.001 & Yes \\
\hline PD vs PSF & & & 0.031 & Yes & & & & & 0.001 & Yes \\
\hline M vs PSF & & & 0.011 & Yes & & & & & 0.001 & Yes \\
\hline
\end{tabular}


Table C.1 (continued).

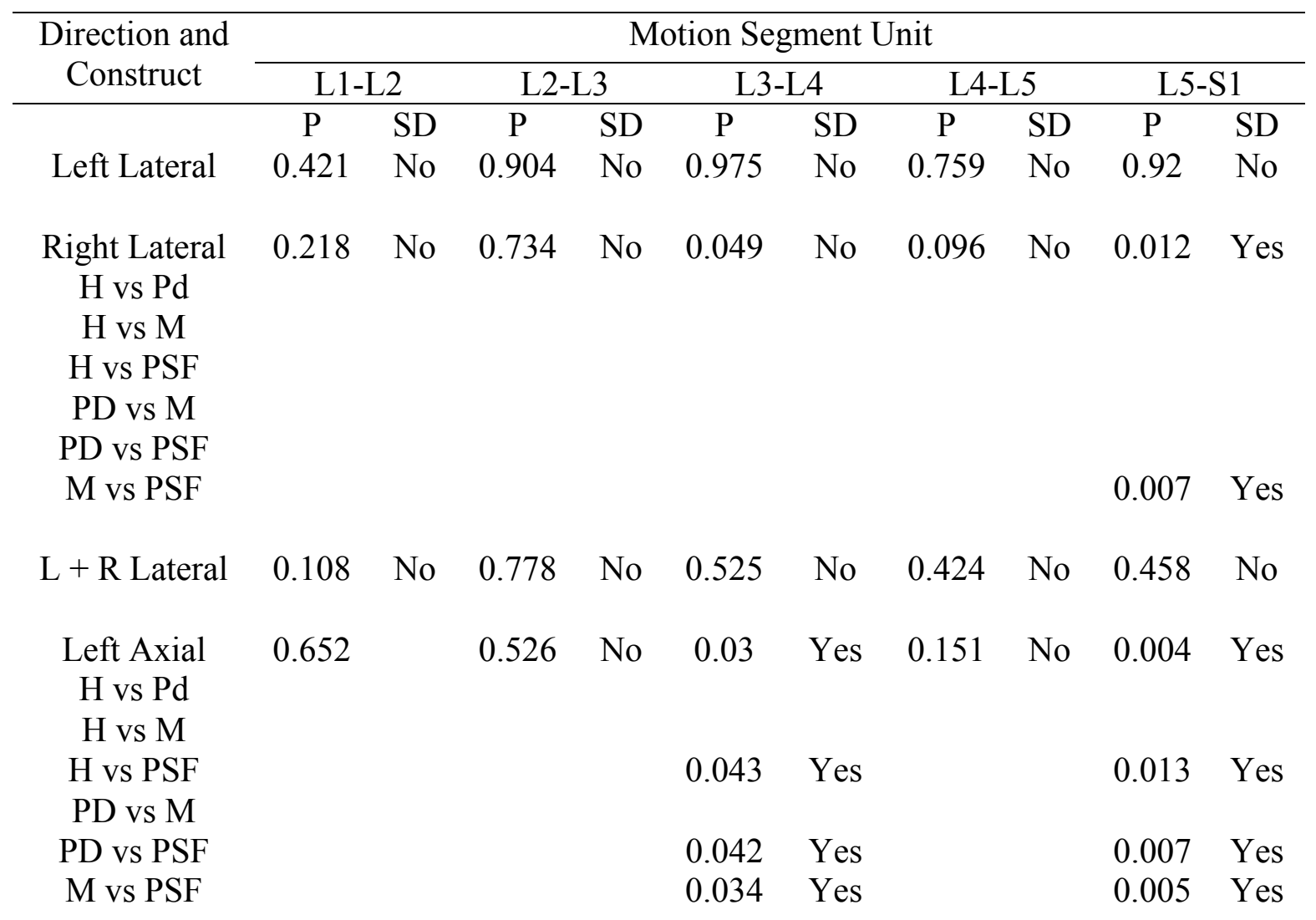


Table C.1 (continued).

\begin{tabular}{|c|c|c|c|c|c|c|c|c|c|c|}
\hline \multirow{2}{*}{$\begin{array}{c}\text { Direction and } \\
\text { Construct } \\
\text { Right Axial }\end{array}$} & \multicolumn{10}{|c|}{ Motion Segment Unit } \\
\hline & \multicolumn{2}{|c|}{ L1-L2 } & \multicolumn{2}{|c|}{ L2-L3 } & \multicolumn{2}{|c|}{ L3-L4 } & \multicolumn{2}{|c|}{ L4-L5 } & \multicolumn{2}{|c|}{ L5-S1 } \\
\hline $\begin{array}{c}\text { Right Axial } \\
\text { H vs Pd }\end{array}$ & 0.88 & No & 0.067 & No & 0.002 & Yes & 0.535 & No & 0.002 & Yes \\
\hline H vs M & & & & & & & & & 0.027 & Yes \\
\hline $\mathrm{H}$ vs PSF & & & & & & 0.008 & Yes & & & \\
\hline PD vs $\mathrm{M}$ & & & & & & & & & & \\
\hline PD vs PSF & & & & & & 0.018 & Yes & & 0.012 & Yes \\
\hline M vs PSF & & & & & & 0.002 & Yes & & 0.003 & Yes \\
\hline $\begin{array}{c}\mathrm{L}+\mathrm{R} \text { Axial } \\
\mathrm{H} \text { vs Pd } \\
\mathrm{H} \text { vs M }\end{array}$ & 0.874 & No & 0.299 & No & 0.001 & Yes & 0.225 & No & 0.001 & Yes \\
\hline $\begin{array}{l}\mathrm{H} \text { vs PSF } \\
\mathrm{PD} \text { vs } \mathrm{M}\end{array}$ & & & & & 0.002 & Yes & & & 0.017 & Yes \\
\hline PD vs PSF & & & & & 0.001 & Yes & & & 0.001 & Yes \\
\hline $\mathrm{M}$ vs PSF & & & & & 0.001 & Yes & & & 0.001 & Yes \\
\hline
\end{tabular}

H: Harvested PD: ProDisc-L

M: Maverick

PSF: Pedicle Screw Fixation 
Appendix D: Stiffness Curves 

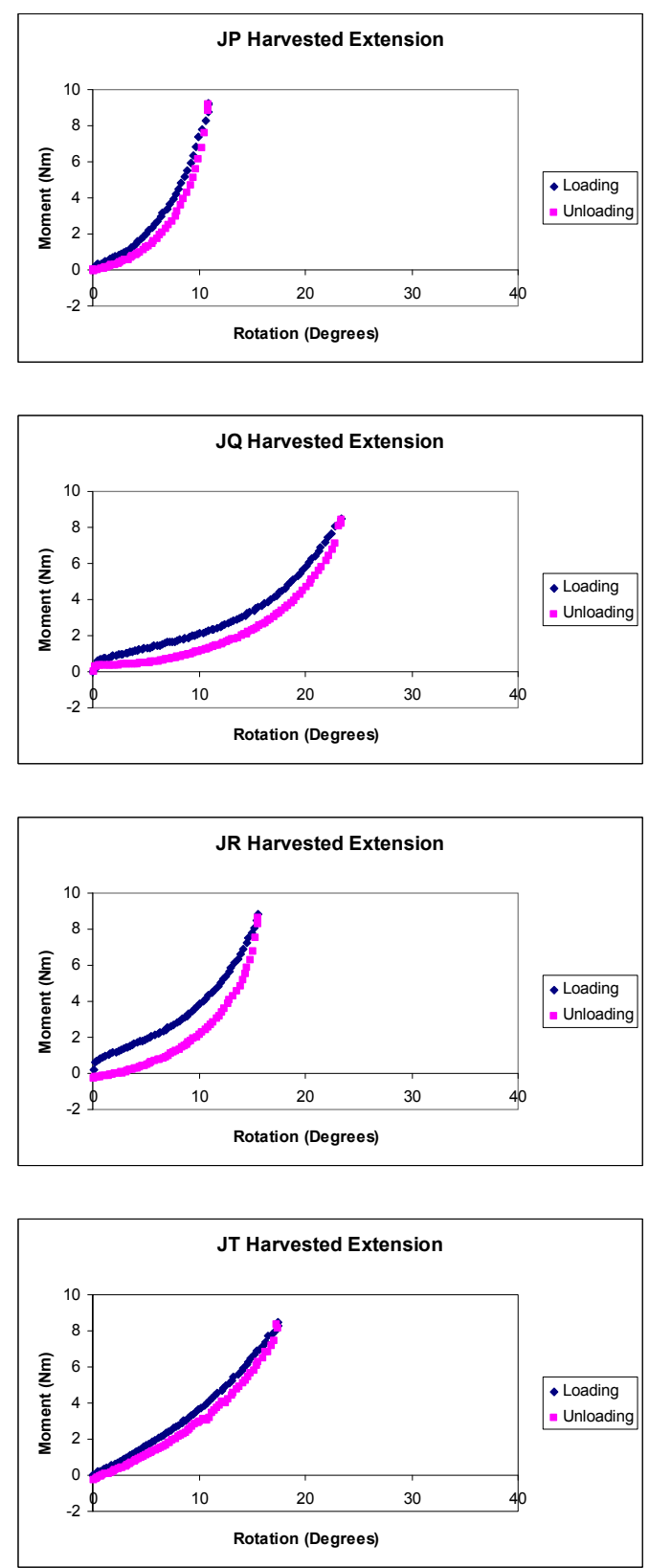
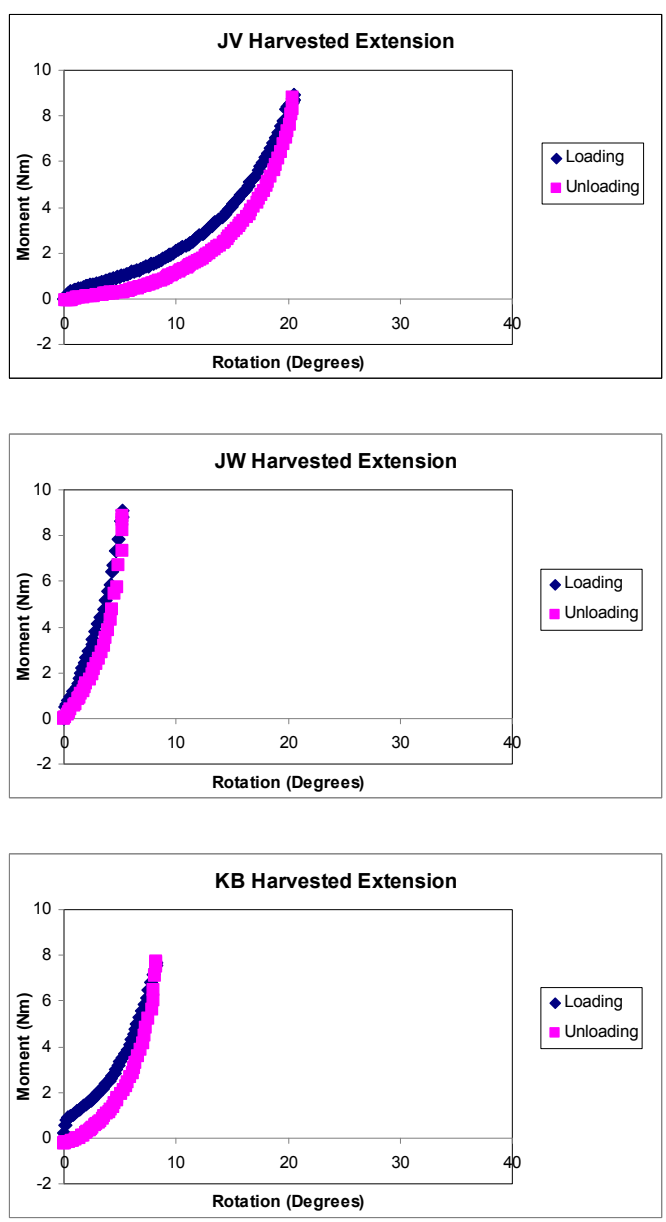

\section{Figure D.1: Harvested Extension—Stiffness Curves.}



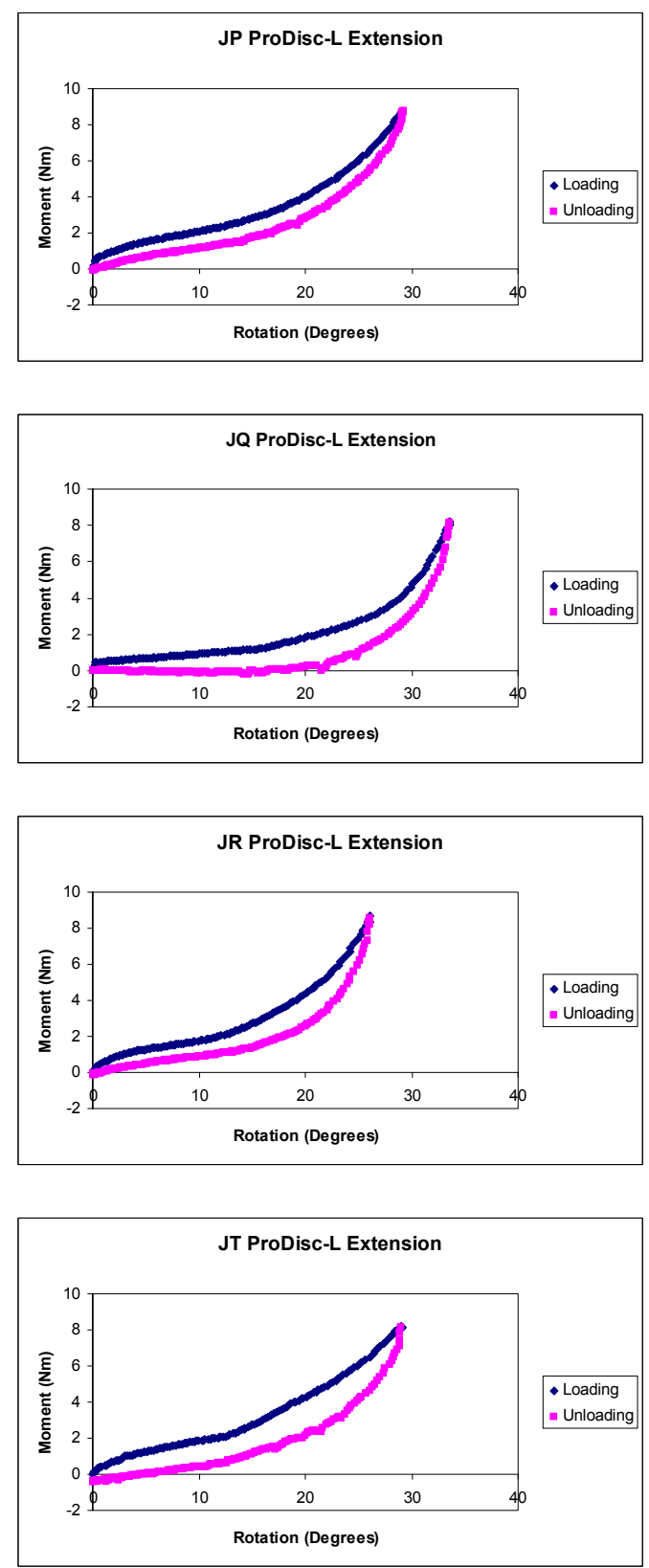
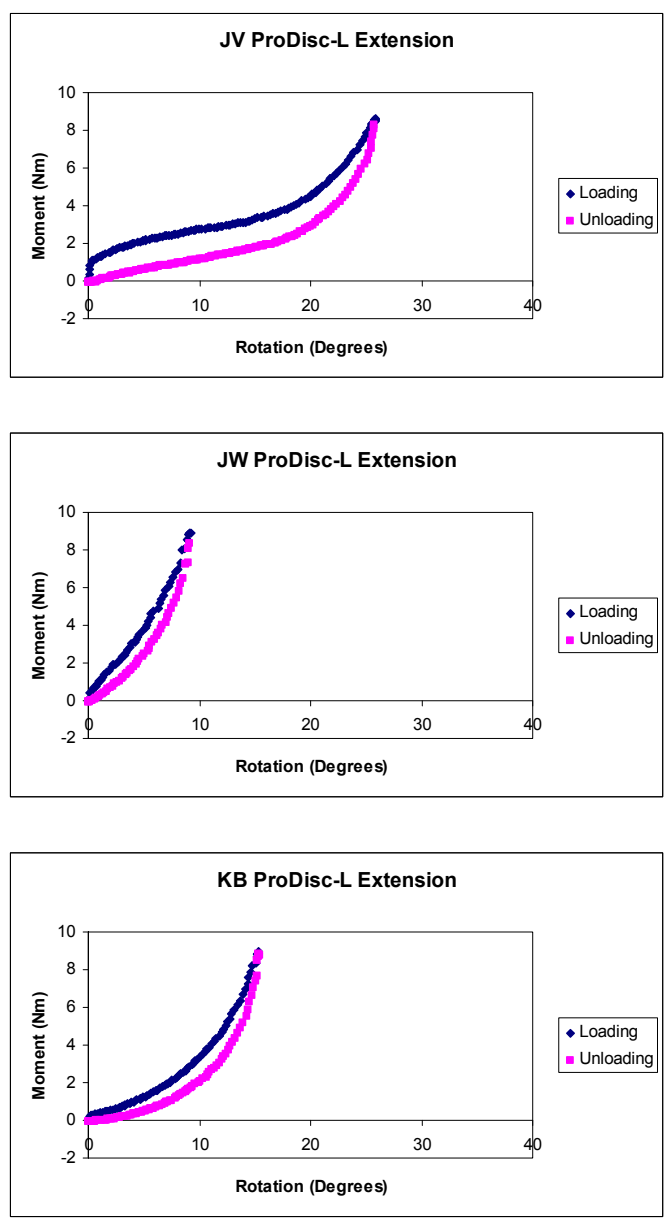

Figure D.2: ProDisc-L Extension-Stiffness Curves. 

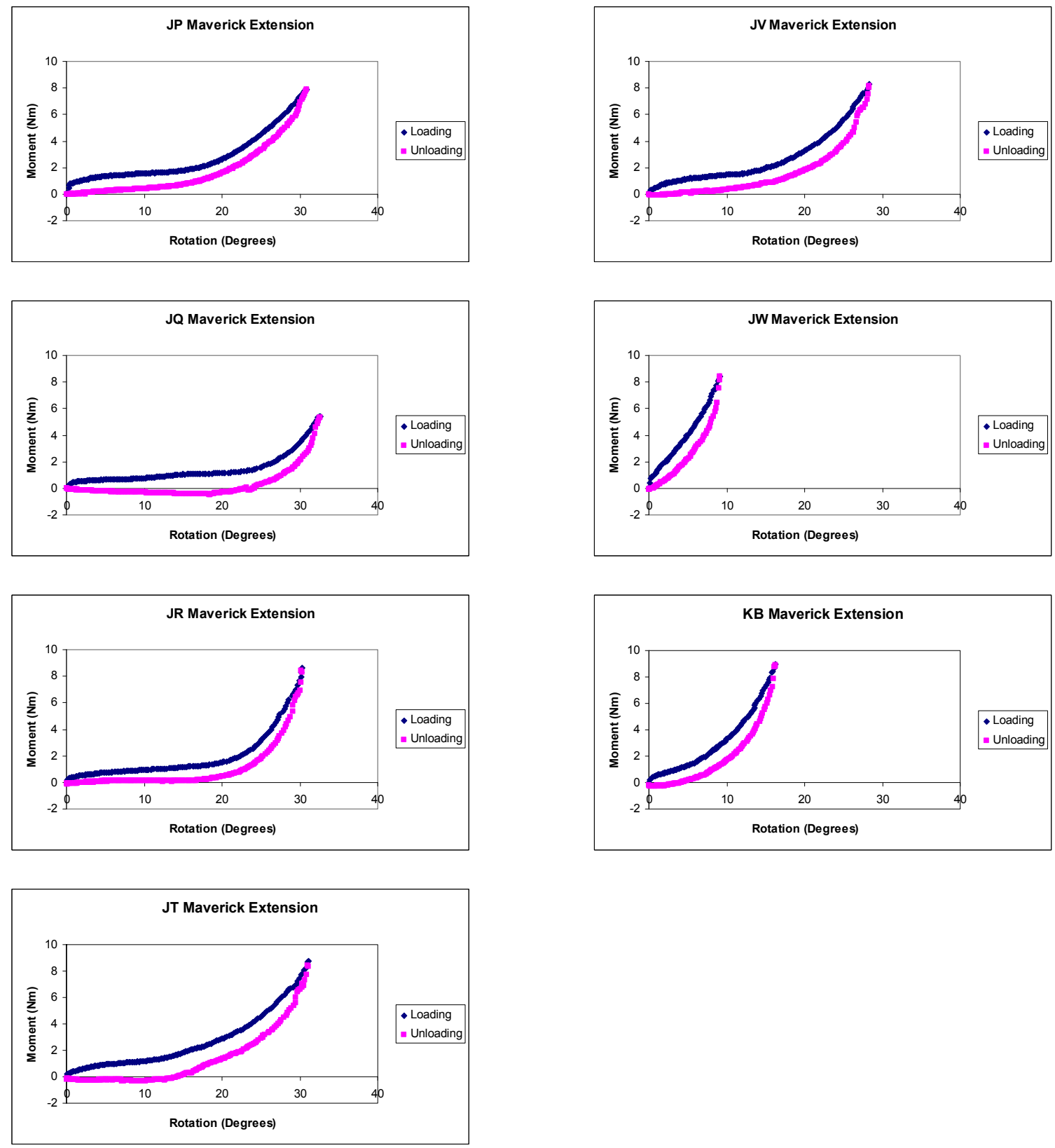

Figure D.3: Maverick Extension—Stiffness Curves. 

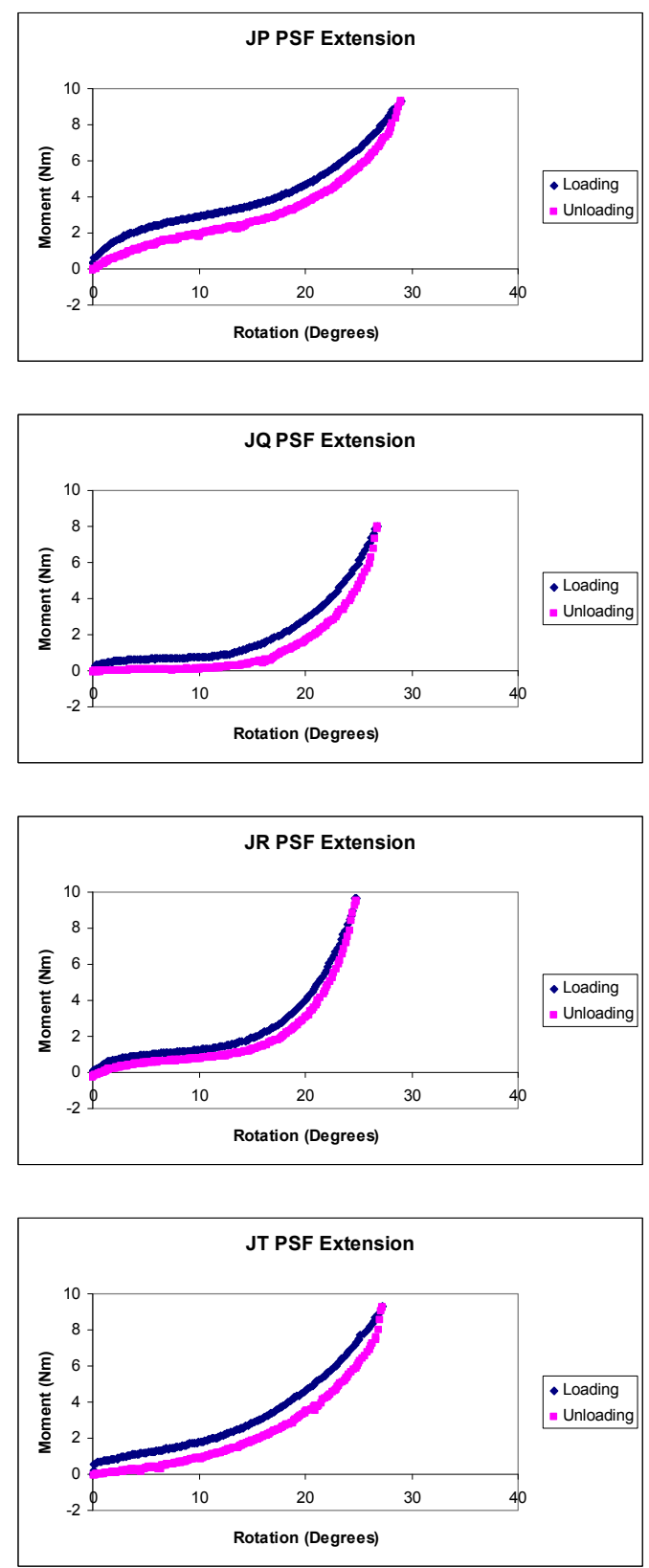
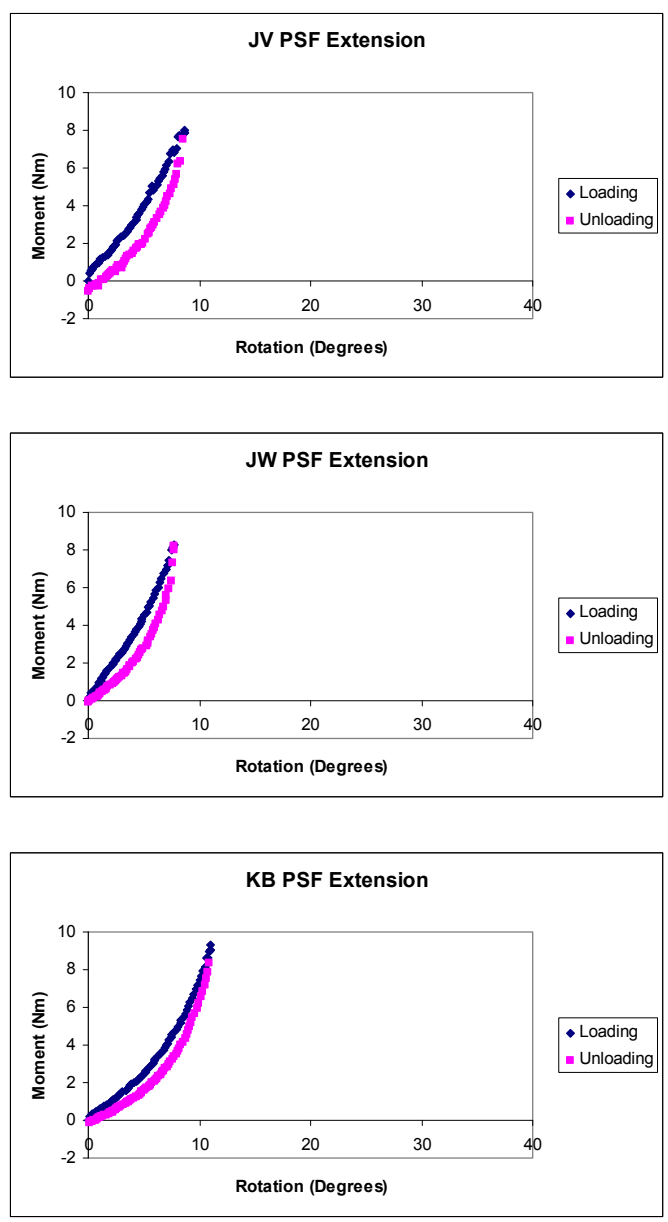

\section{Figure D.4: Pedicle Screw Fixation-Extension Stiffness Curves.}



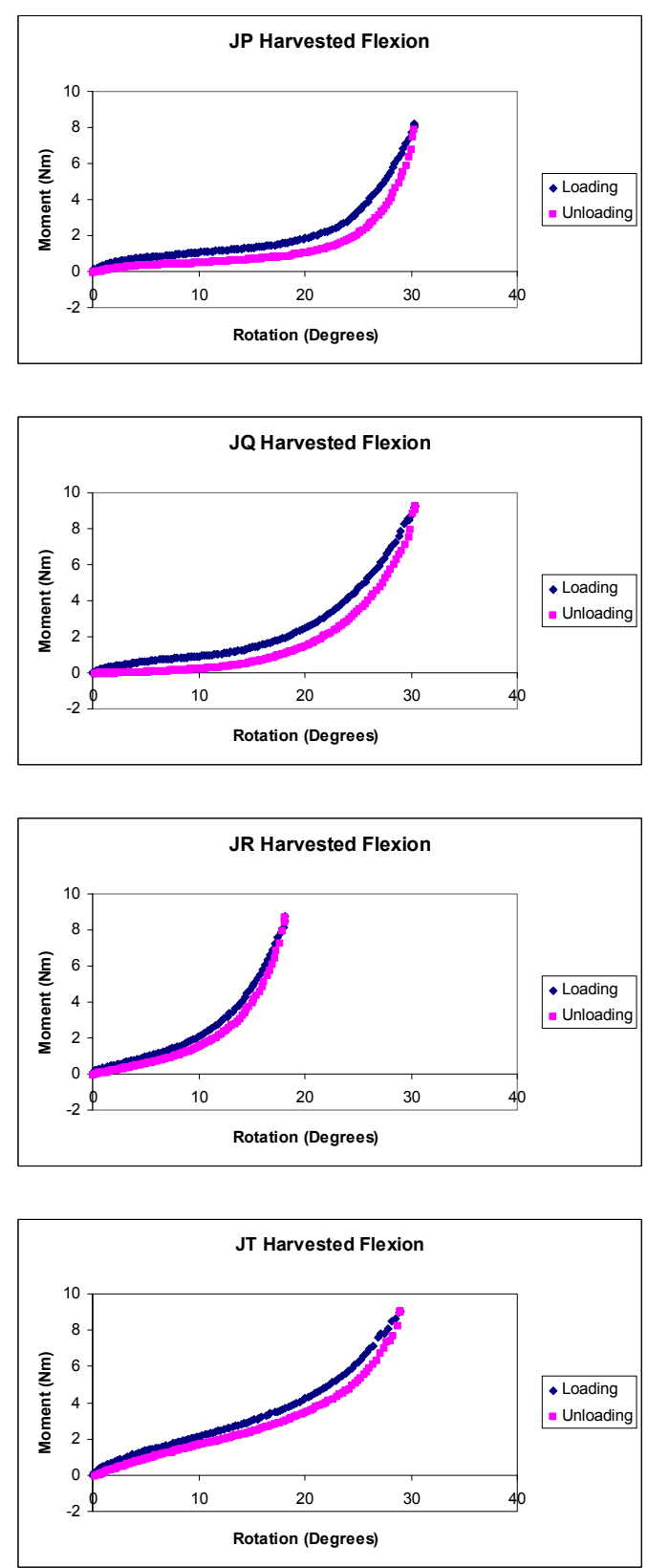
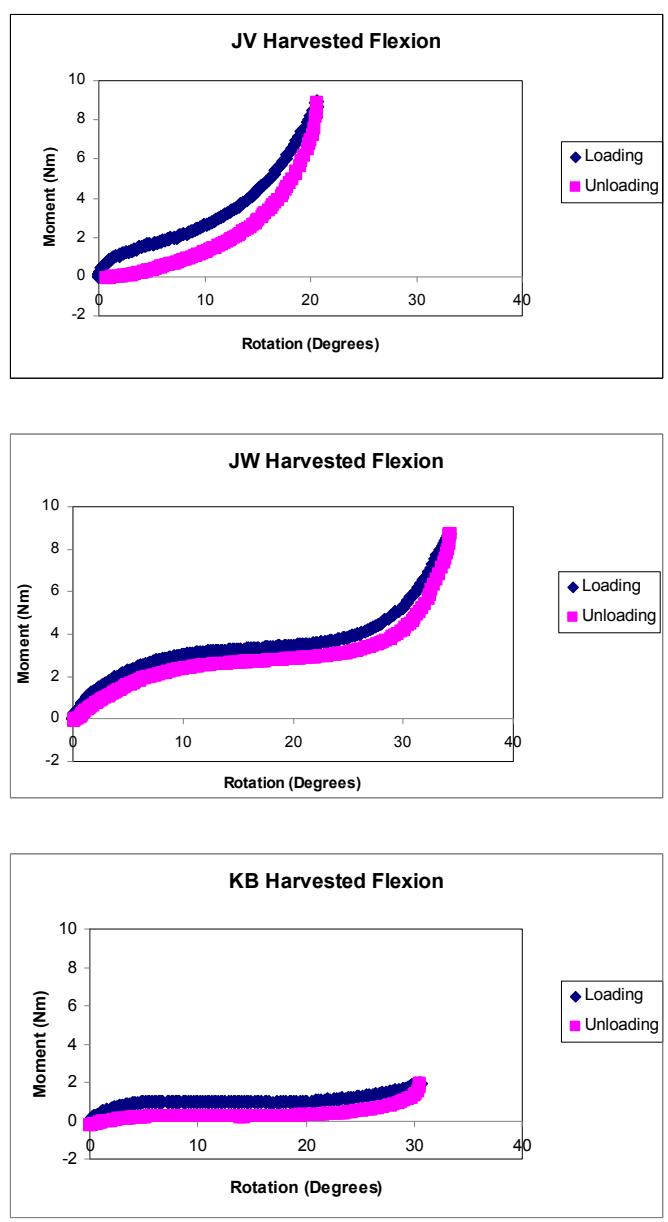

\section{Figure D.5: Harvested Flexion-Stiffness Curves.}



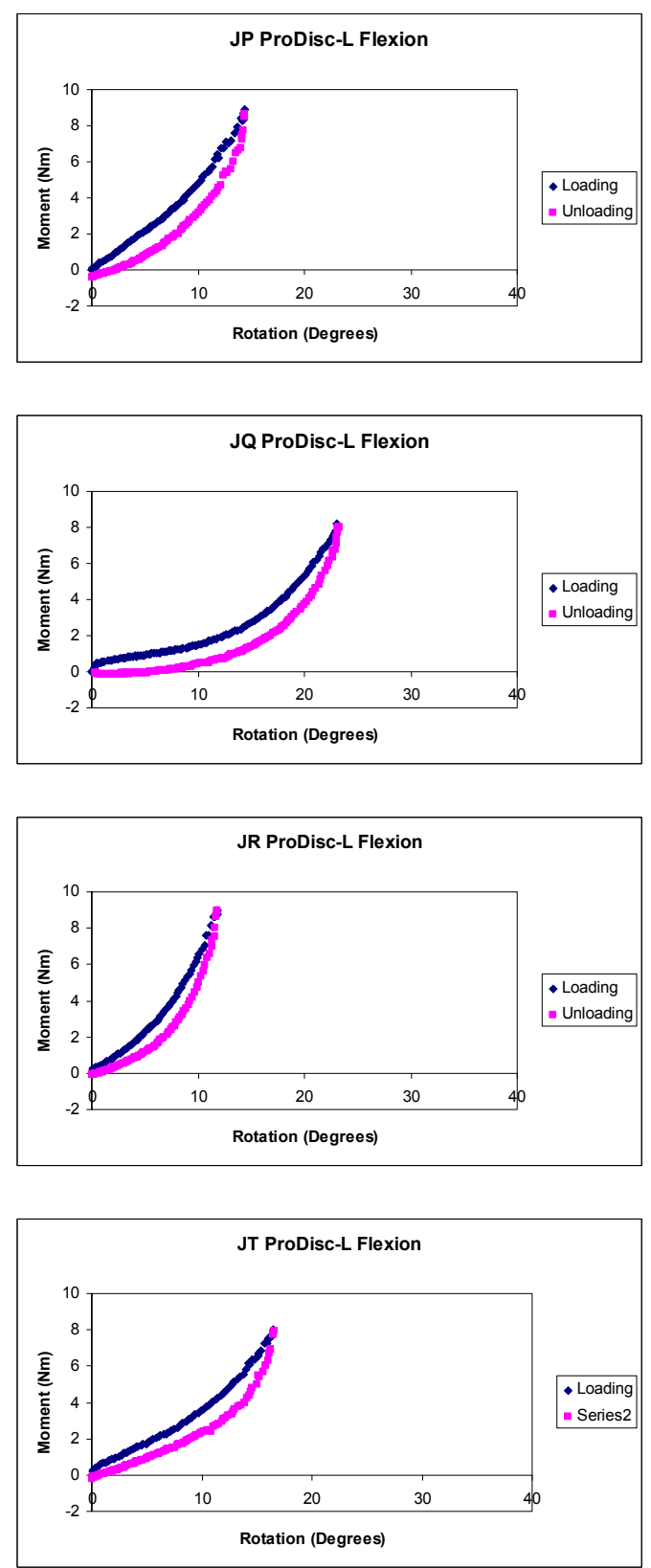
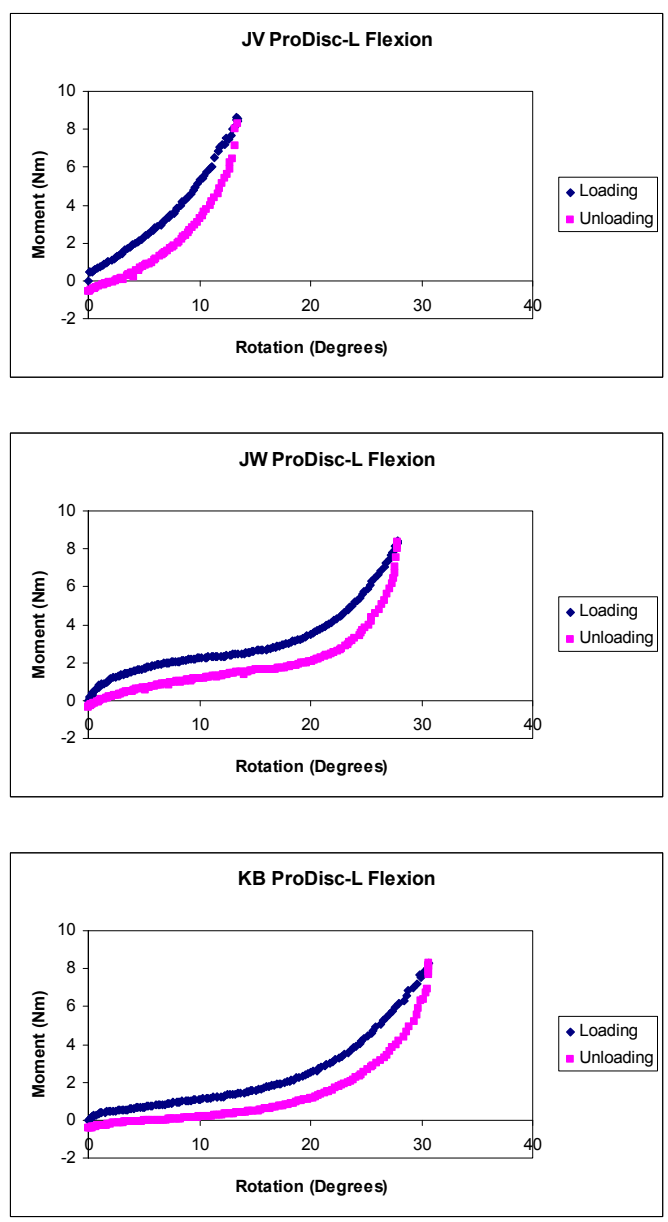

Figure D.6: ProDisc-L Flexion-Stiffness Curves. 

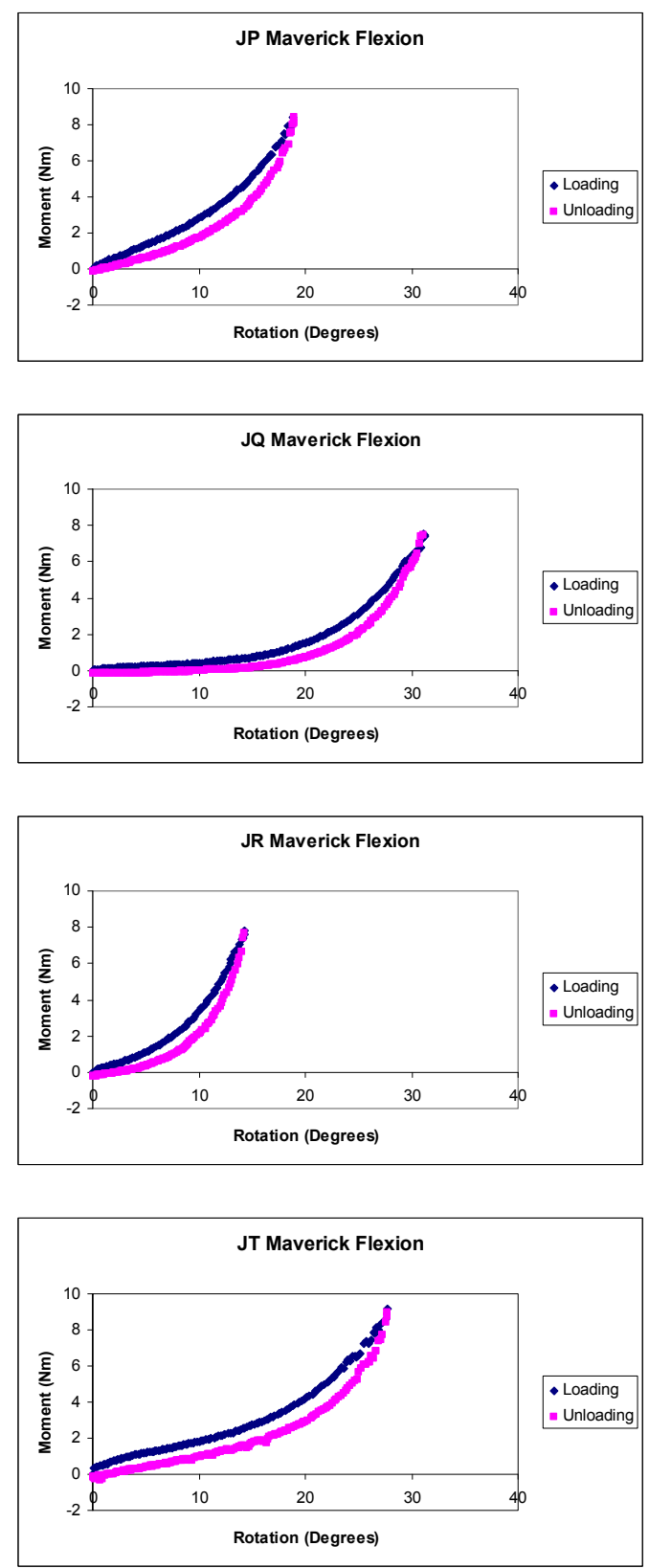
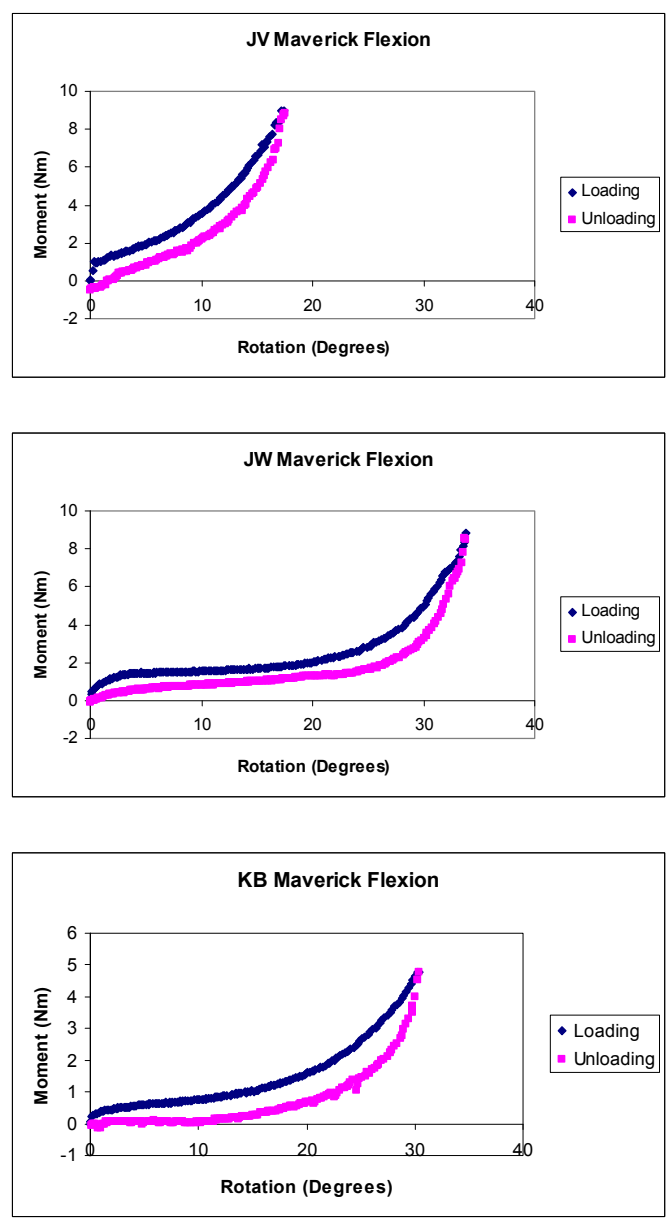

\section{Figure D.7: Maverick Flexion-Stiffness Curves.}



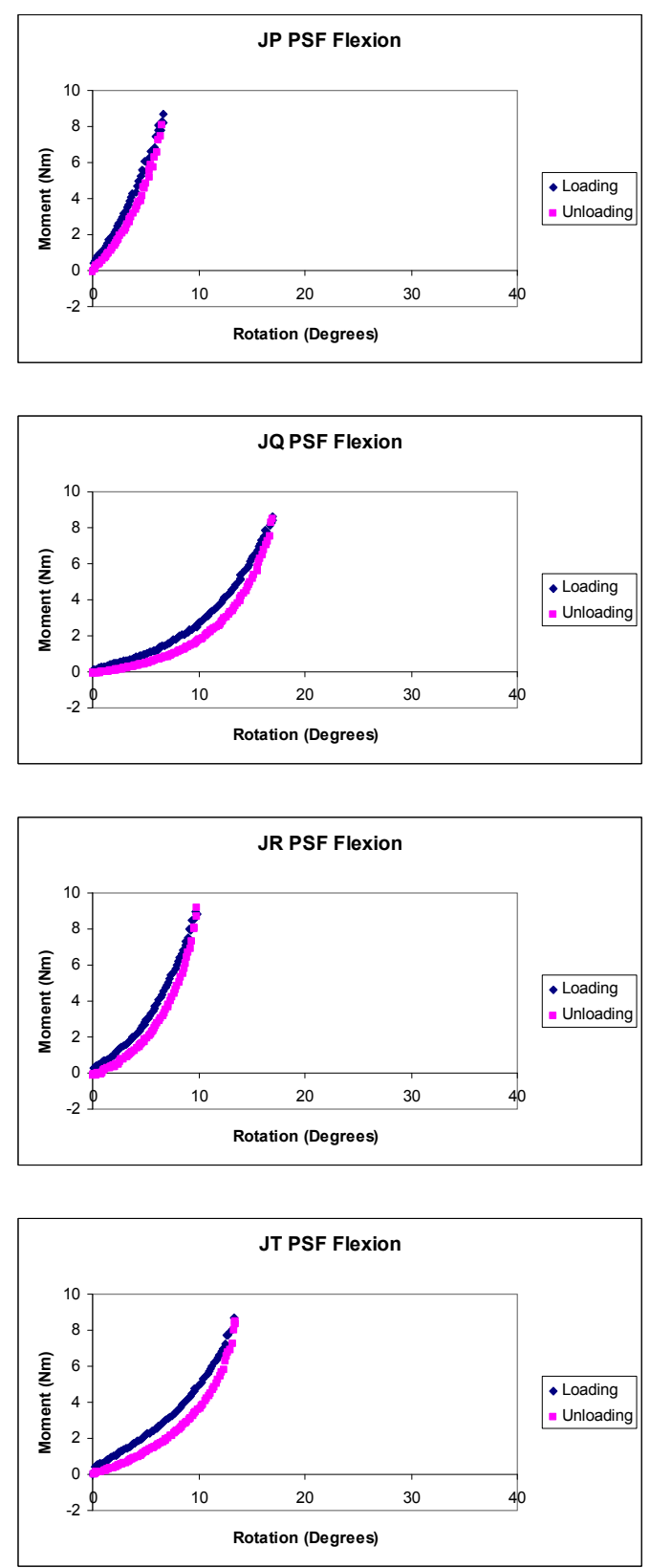
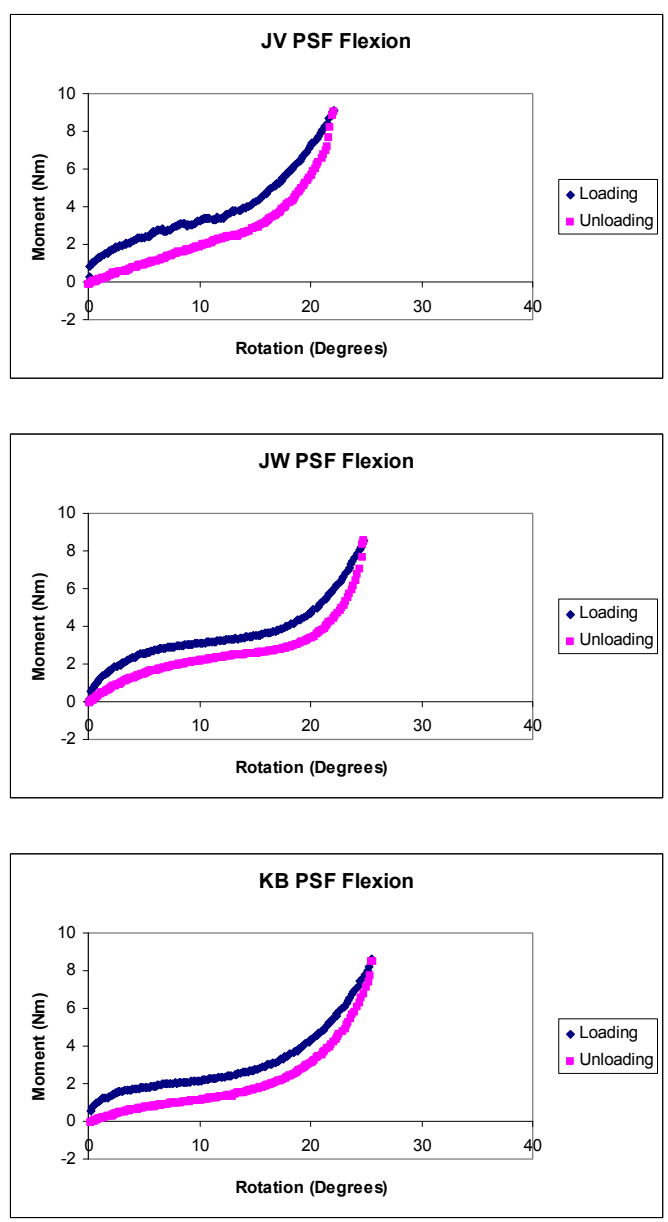

Figure D.8: Pedicle Screw Fixation Flexion-Stiffness Curves. 

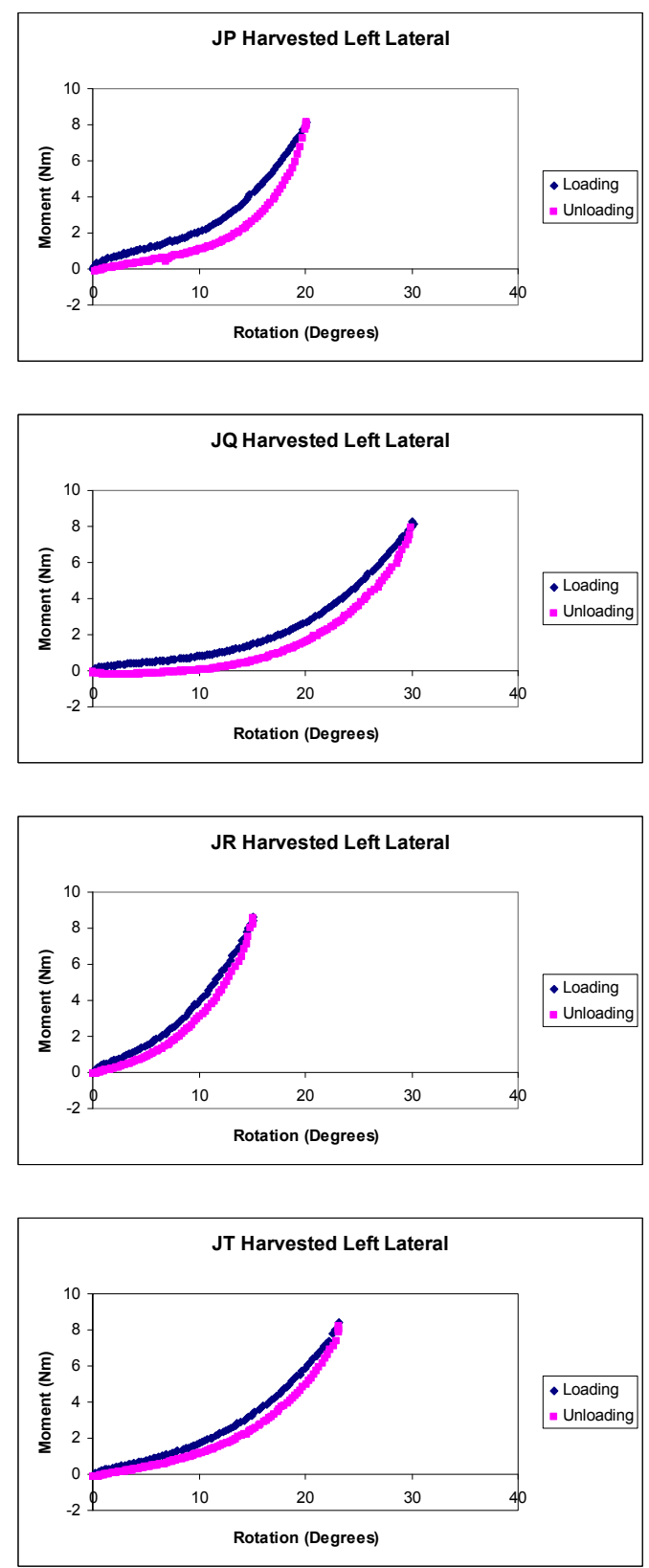
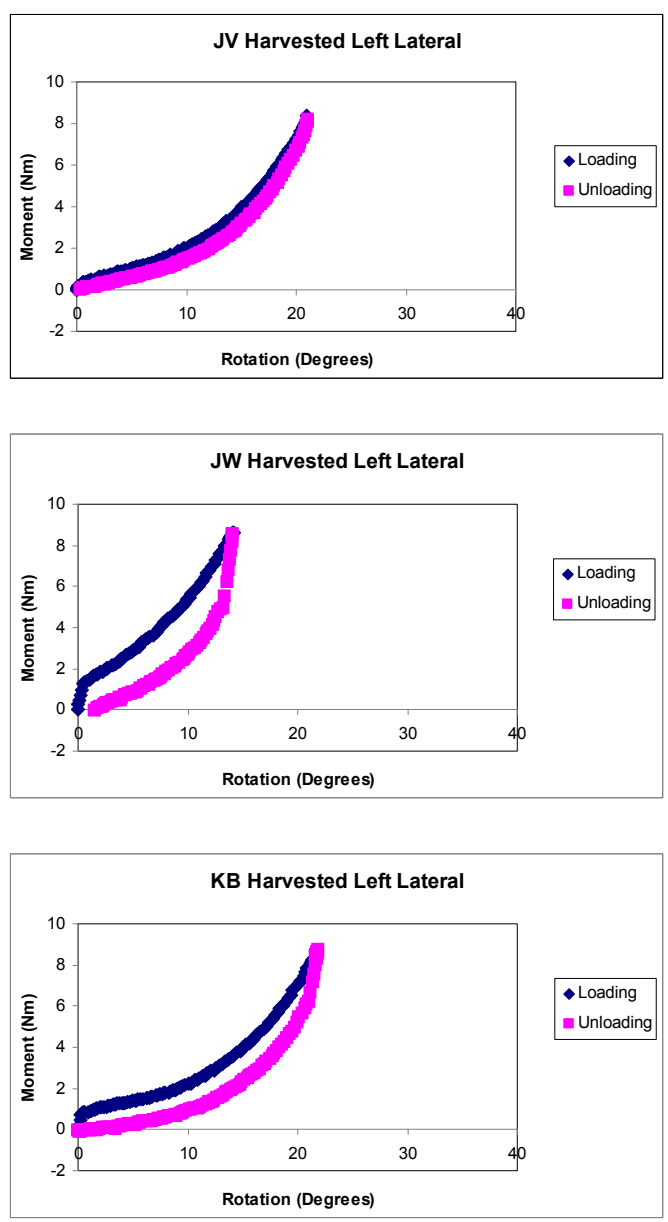

\section{Figure D.9: Harvested Left Lateral—Stiffness Curves.}



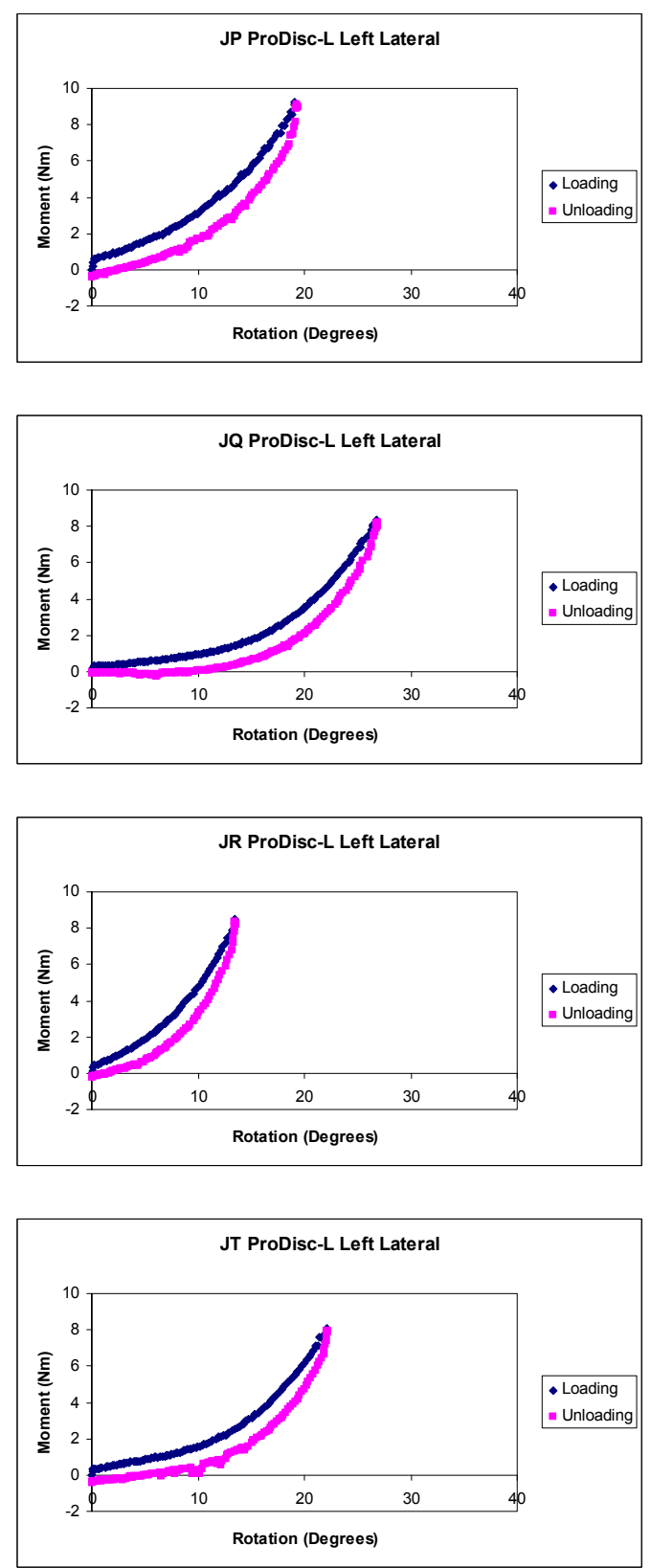
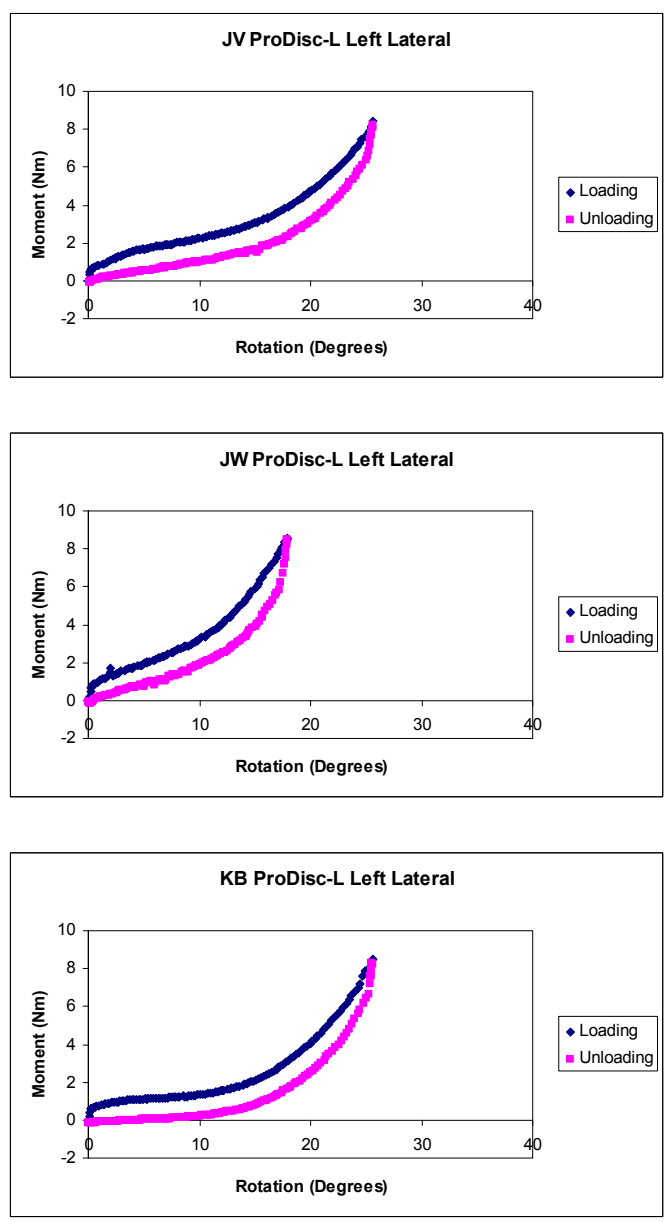

\section{Figure D.10: ProDisc-L Left Lateral-Stiffness Curves.}



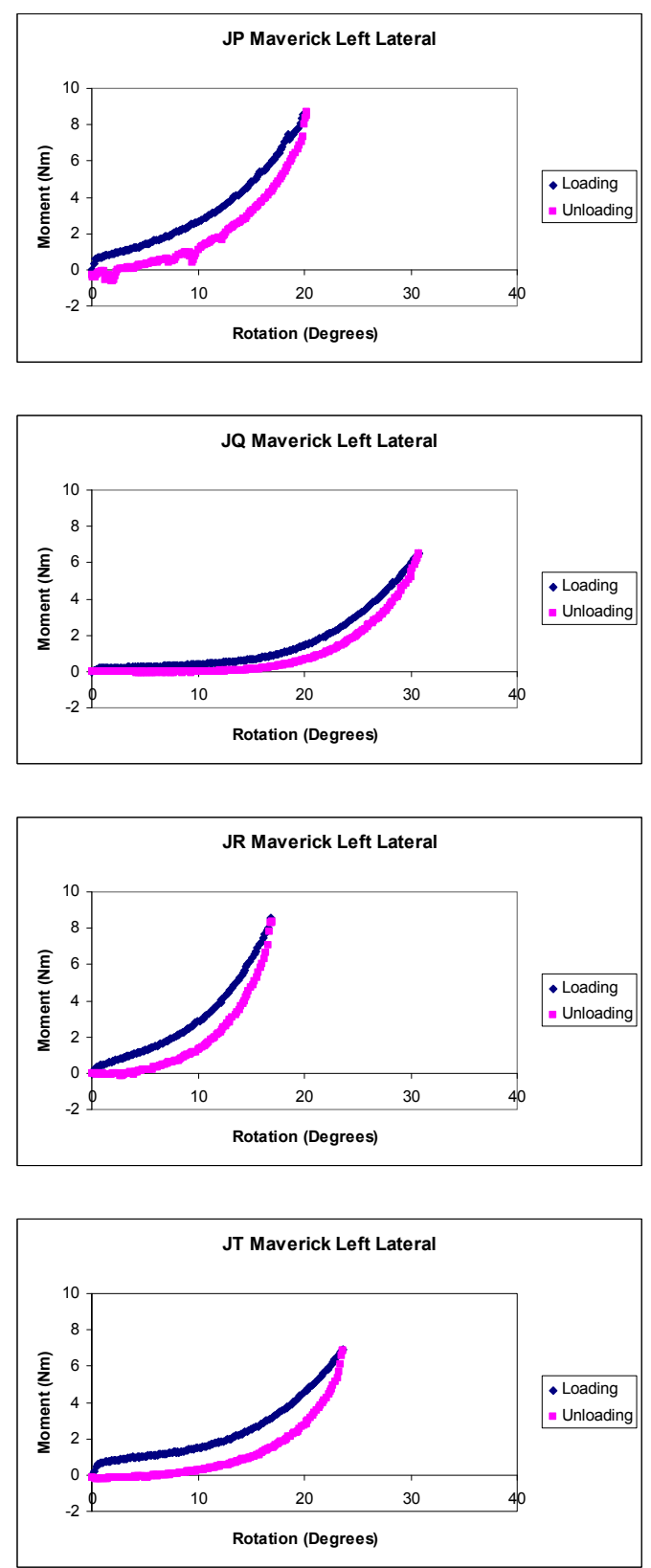
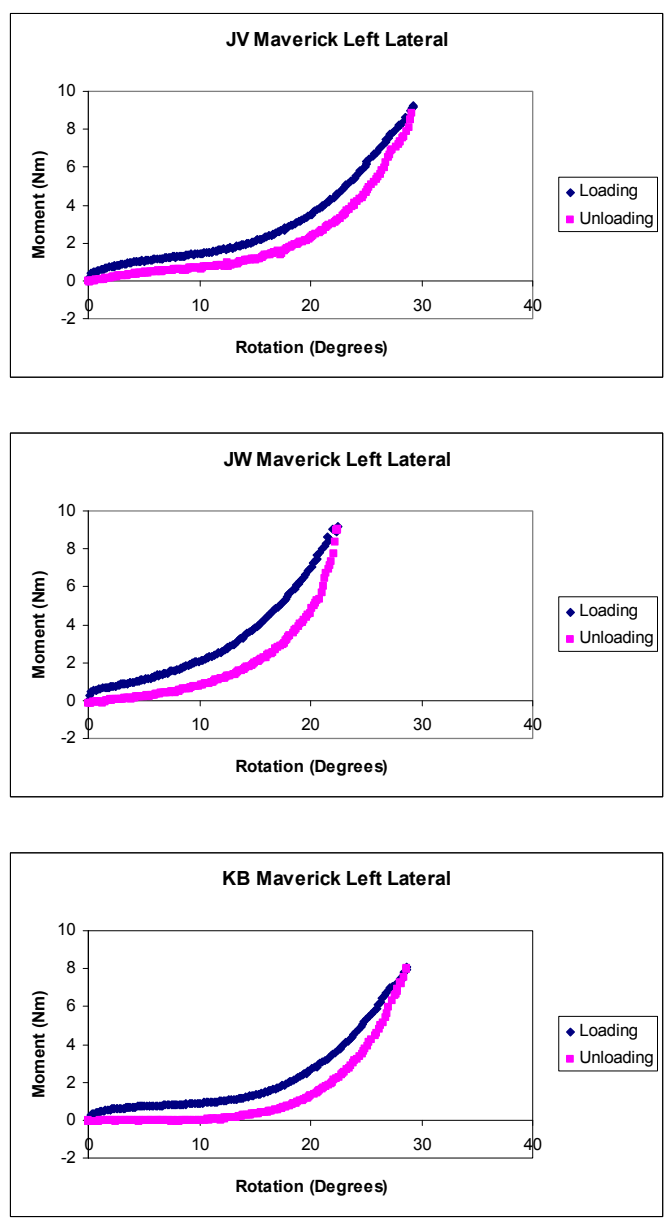

\section{Figure D.11: Maverick Left Lateral—Stiffness Curves.}



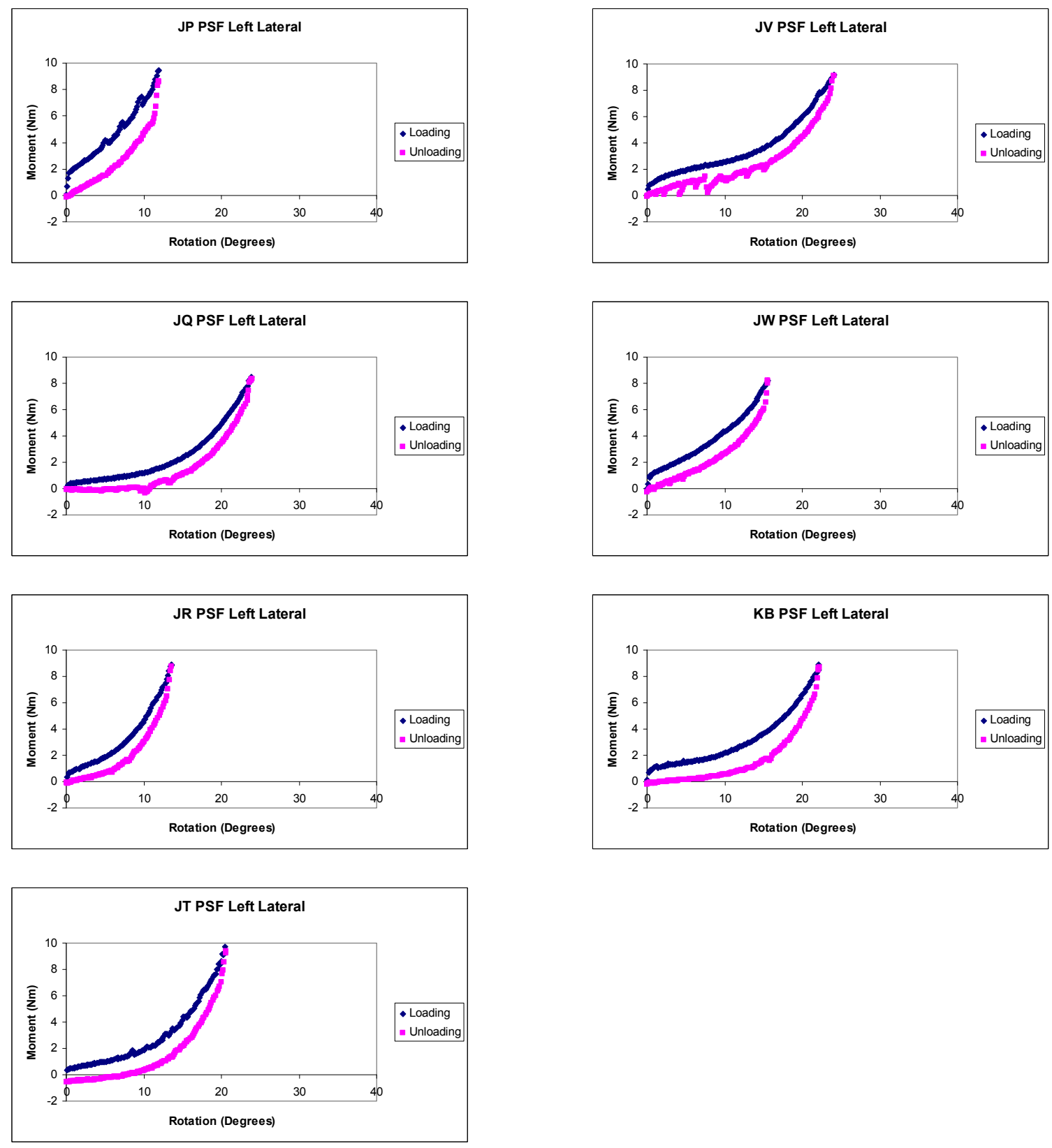

\section{Figure D.12: Pedicle Screw Fixation Left Lateral—Stiffness Curves.}



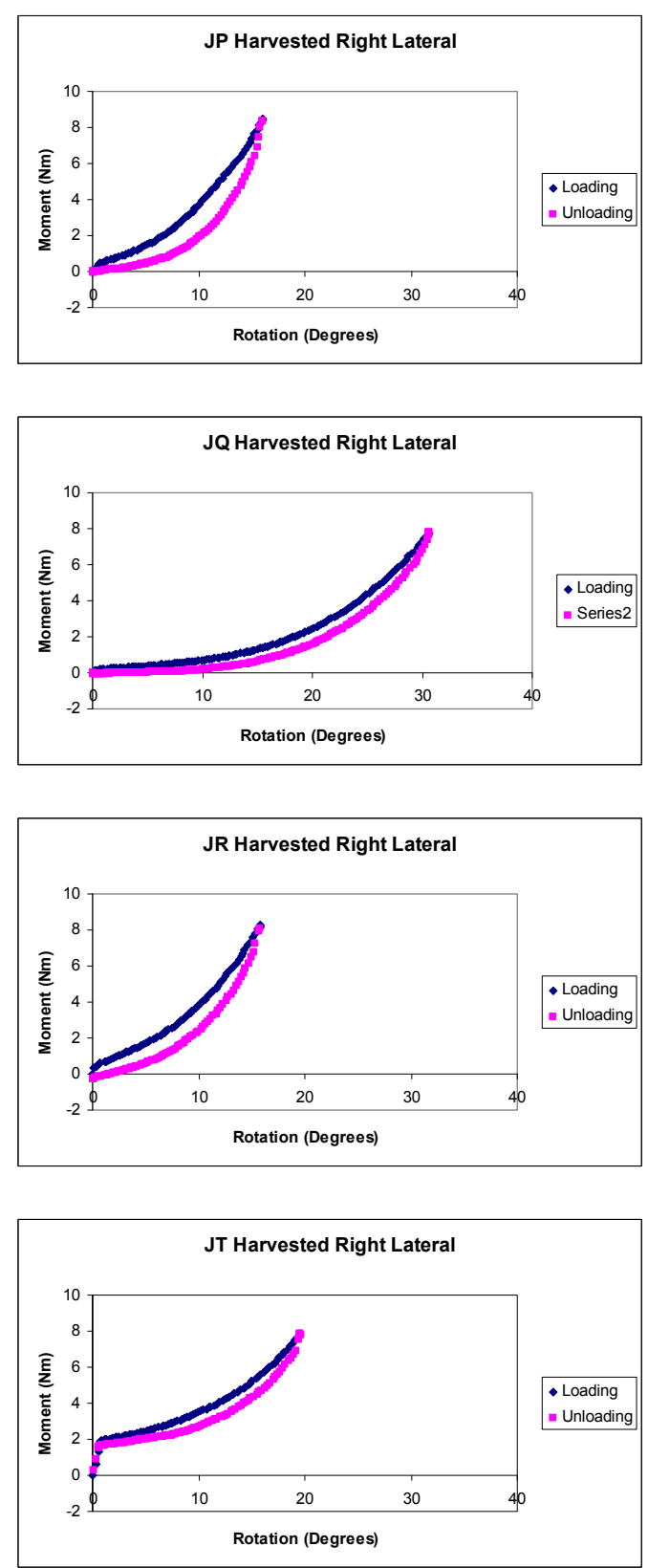
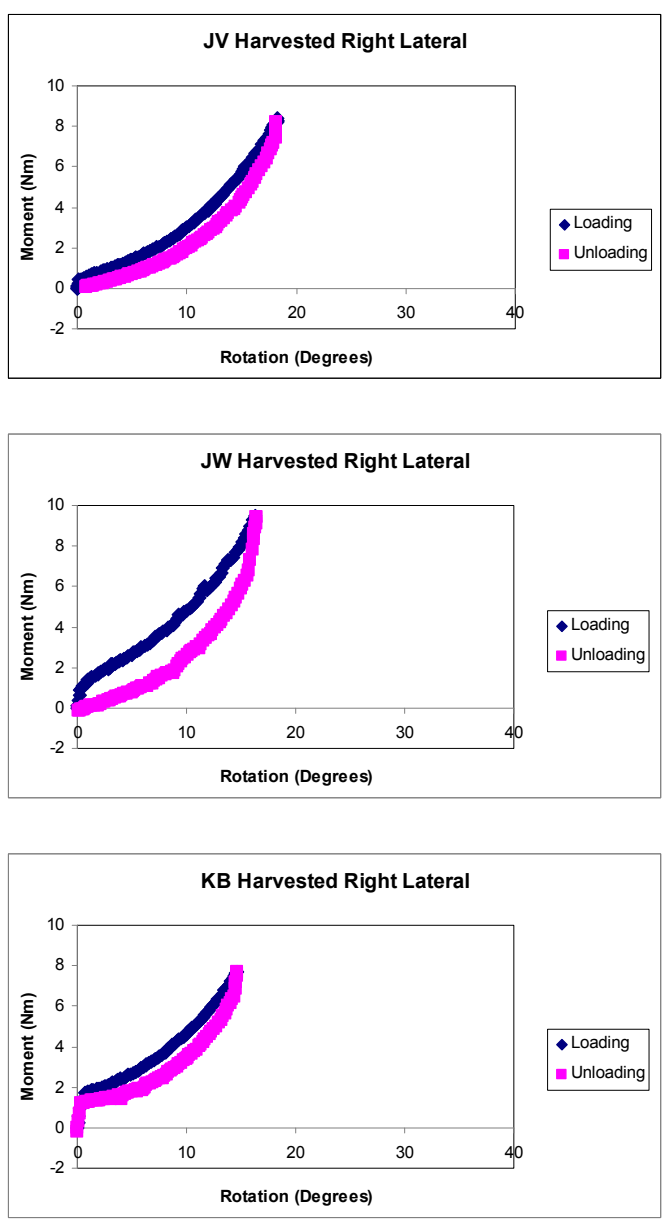

Figure D.13: Harvested Right Lateral—Stiffness Curves. 

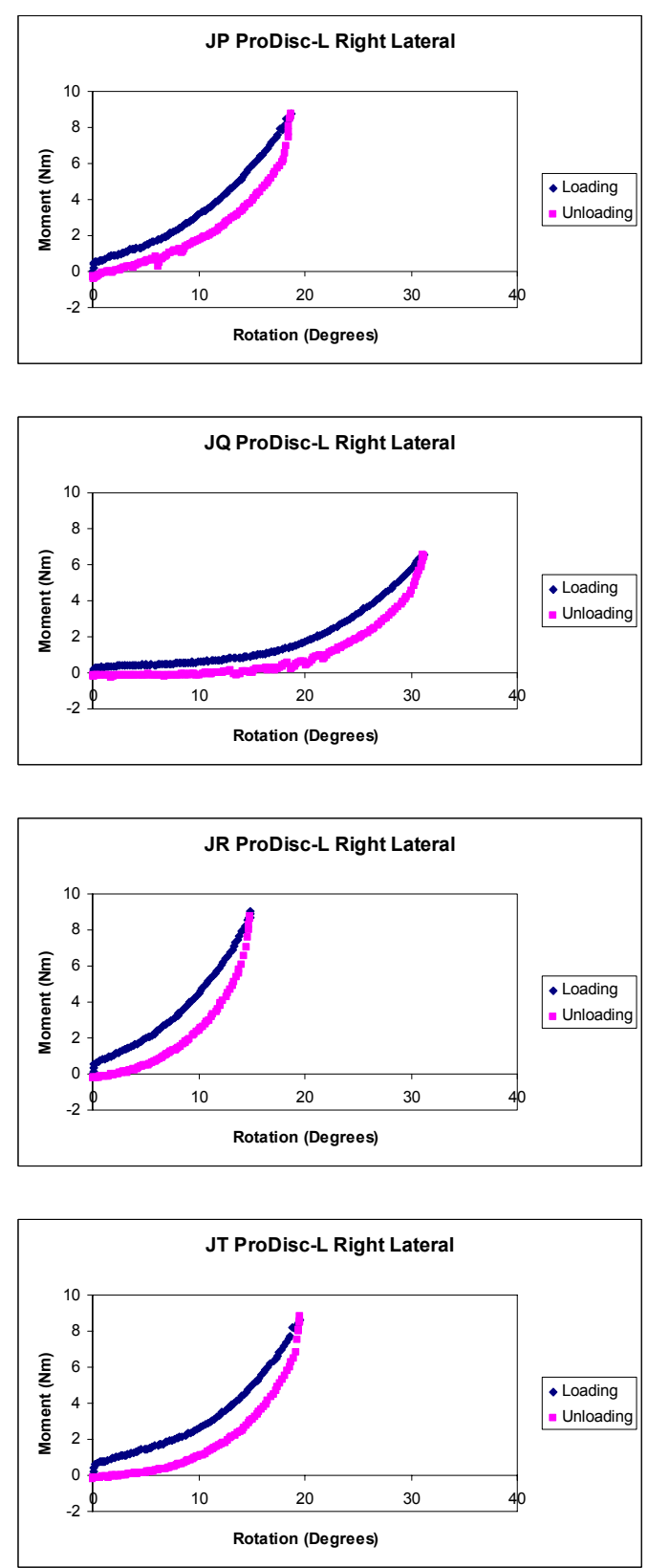
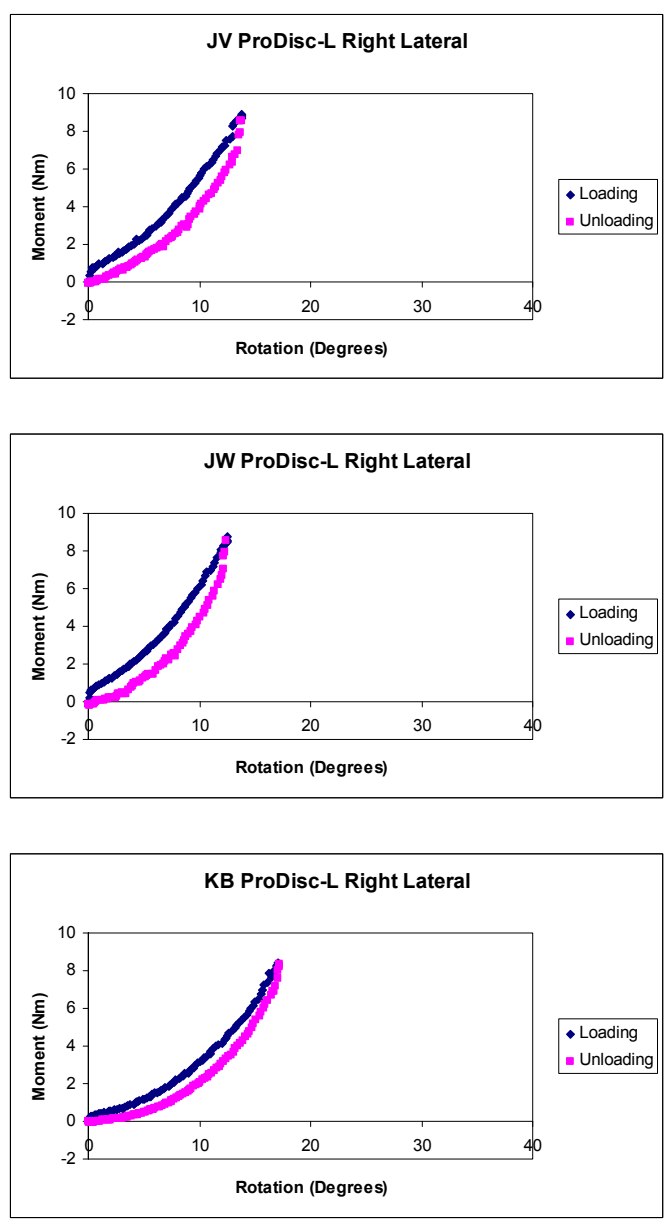

Figure D.14: ProDisc-L Right Lateral—Stiffness Curves. 

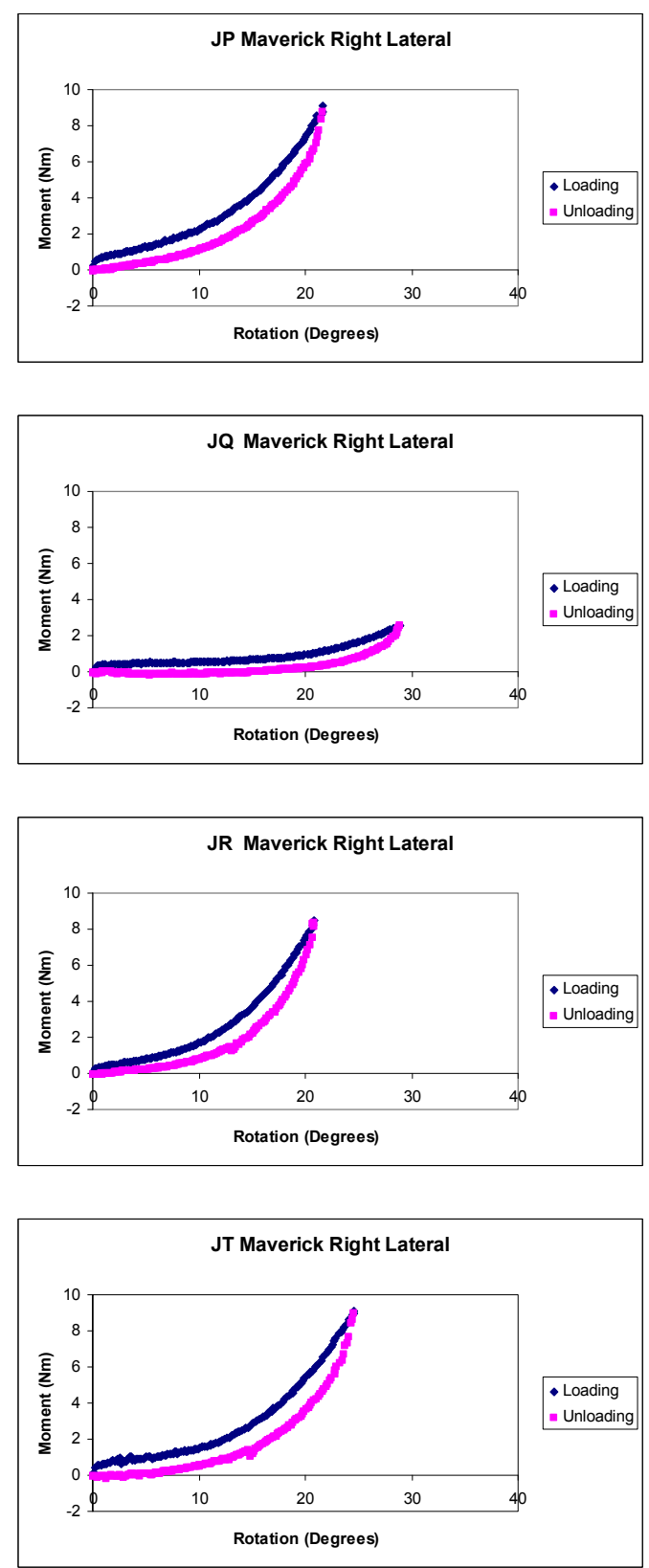
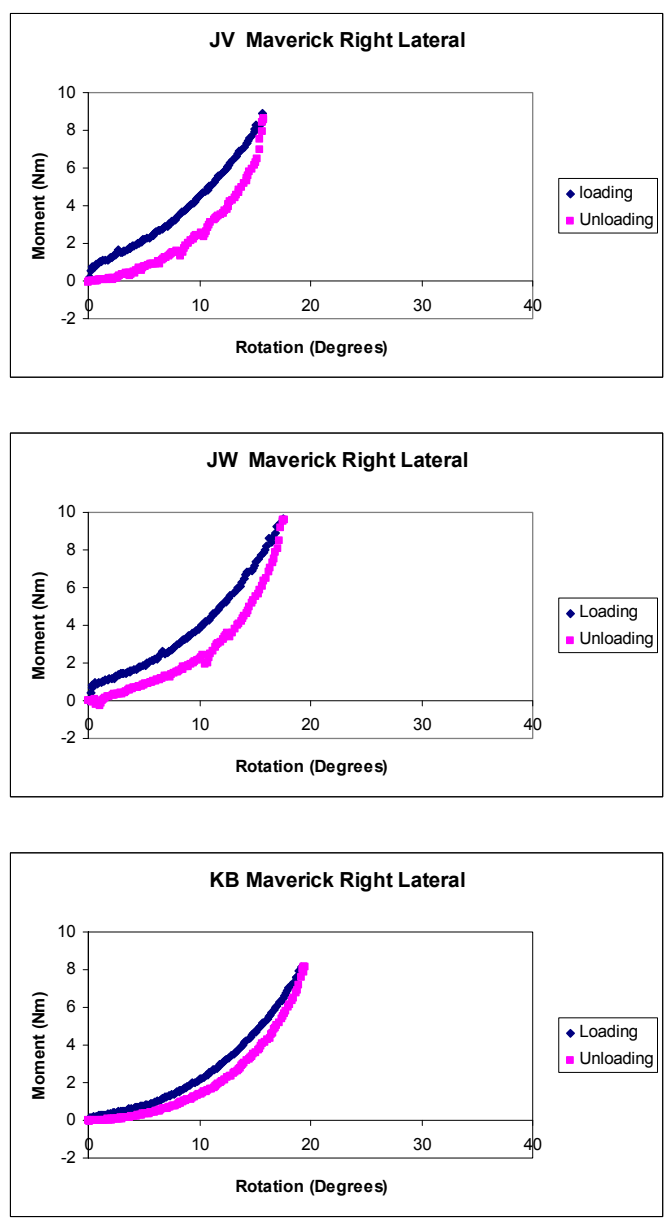

Figure D.15: Maverick Right Lateral—Stiffness Curves. 

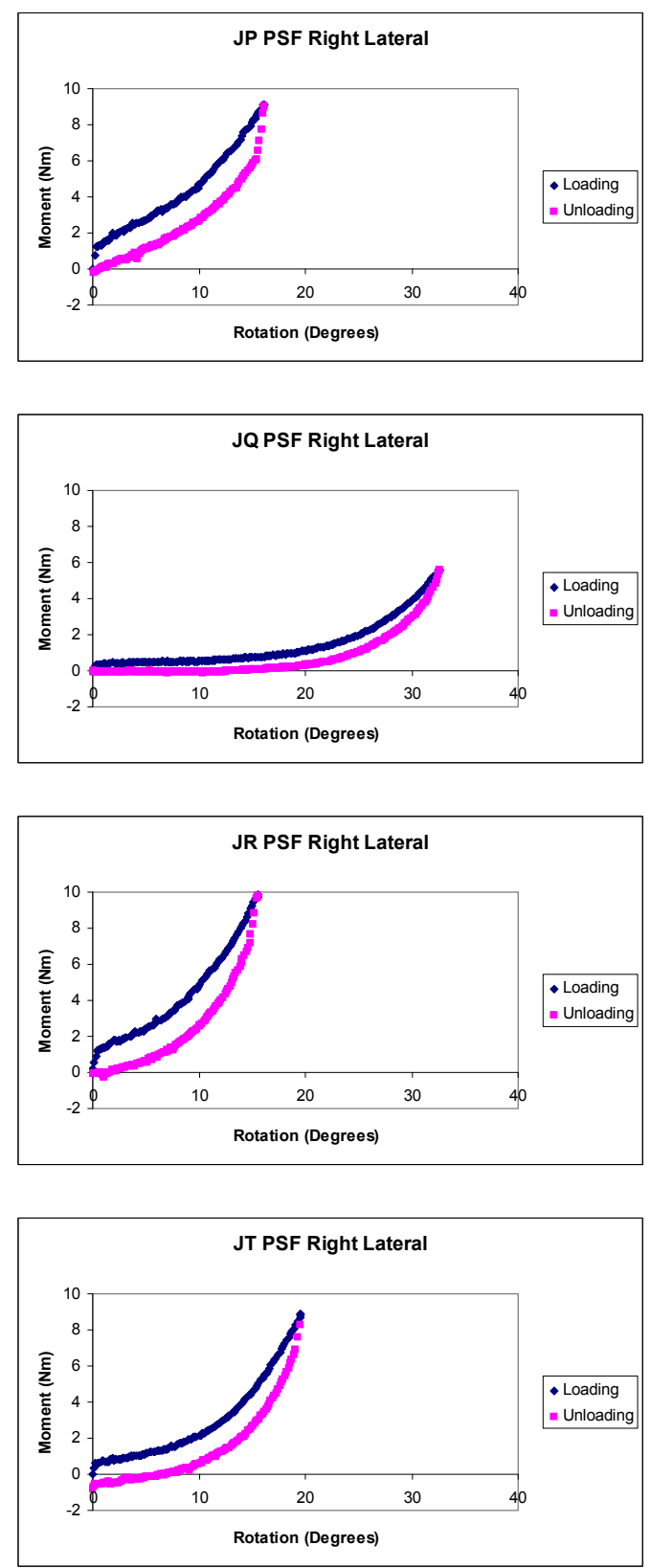
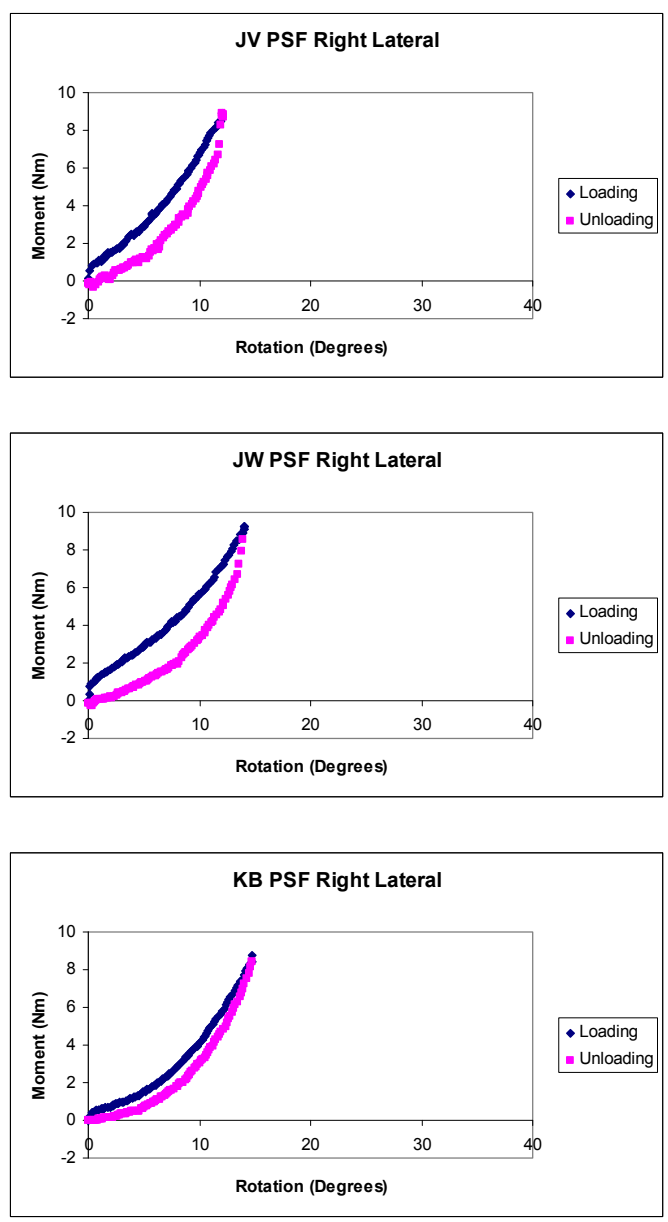

Figure D.16: Pedicle Screw Fixation Right Lateral—Stiffness Curves. 

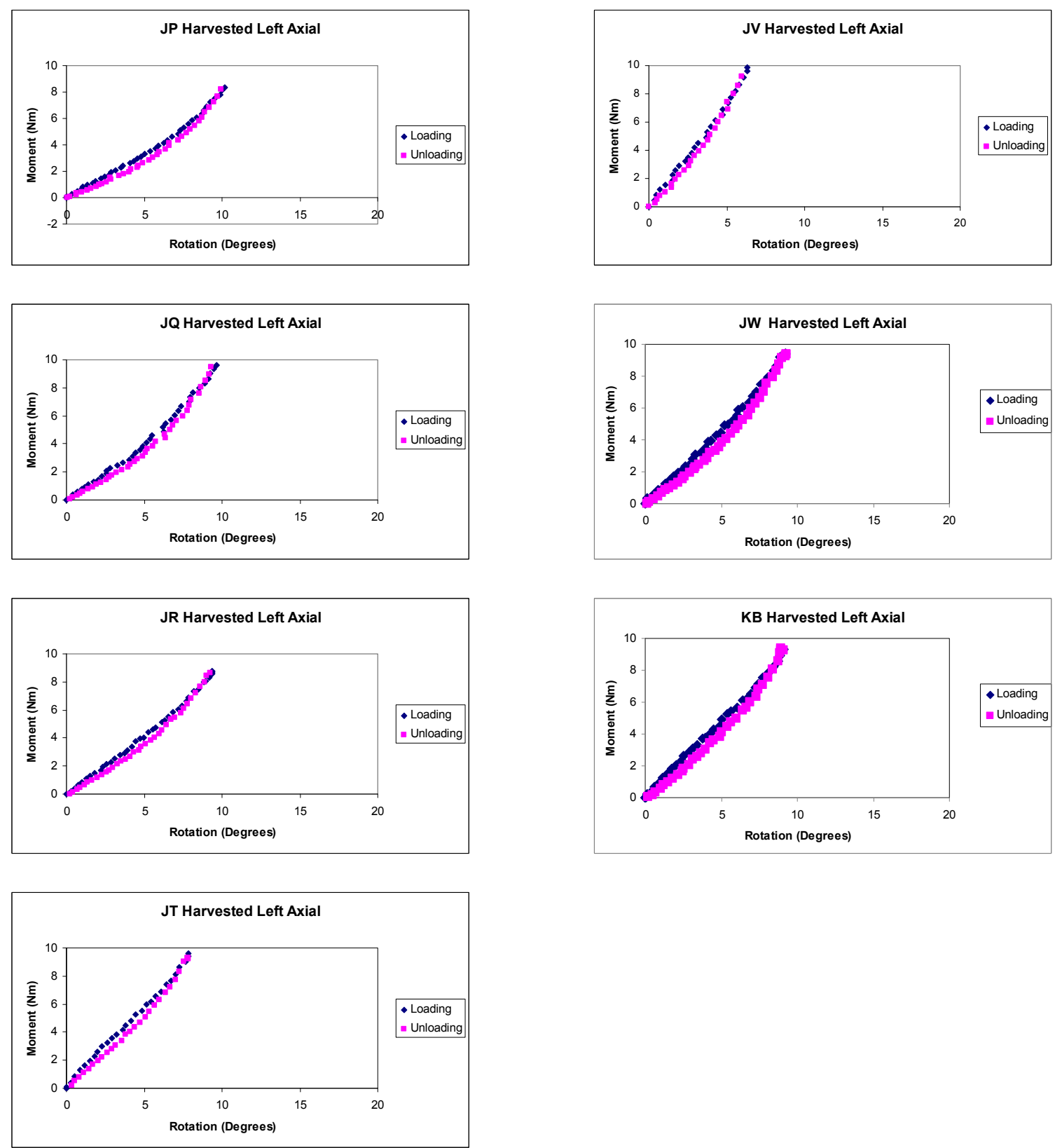

Figure D.17: Harvested Left Axial—Stiffness Curves. 

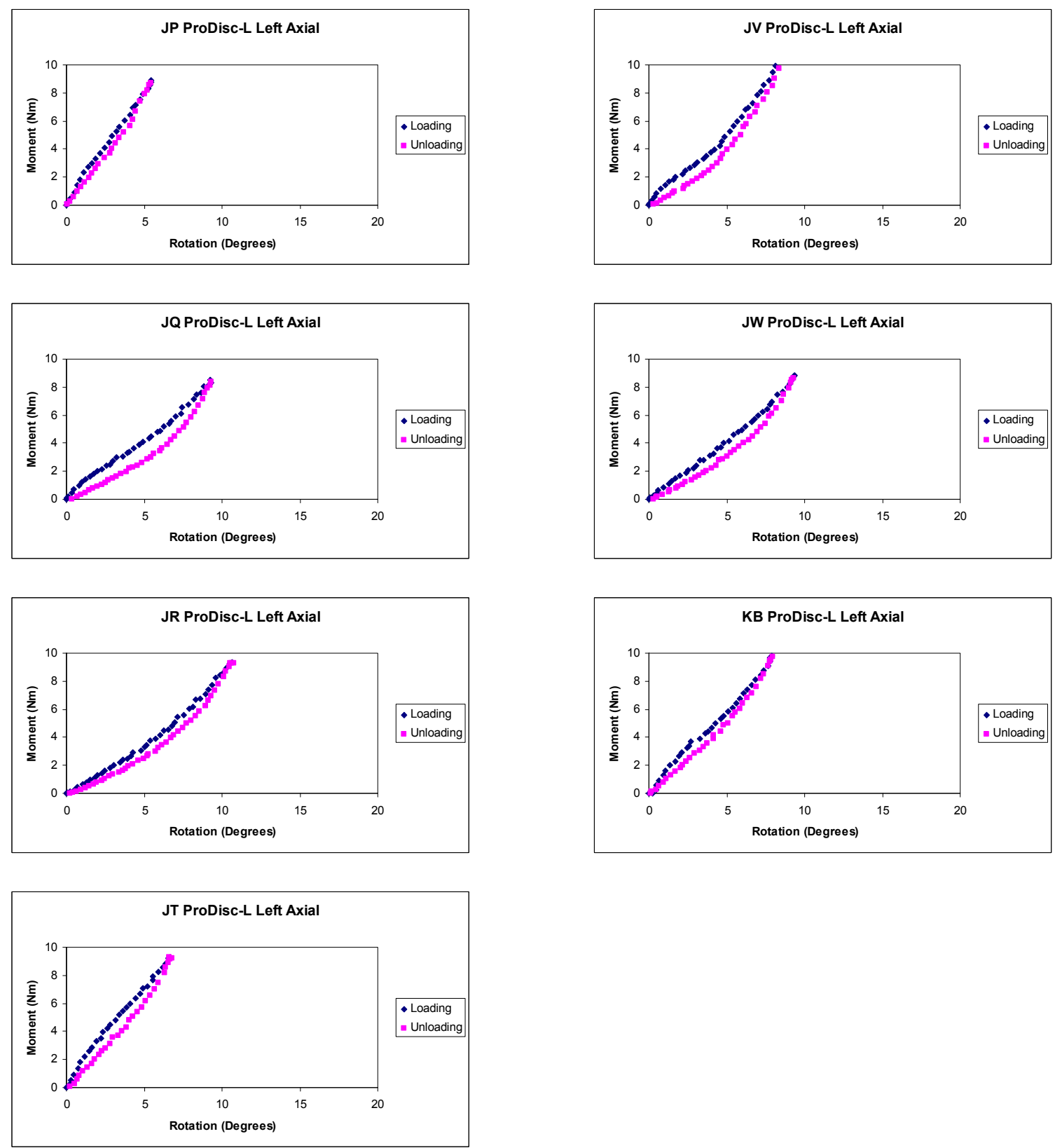

Figure D.18: ProDisc-L Left Axial-Stiffness Curves. 

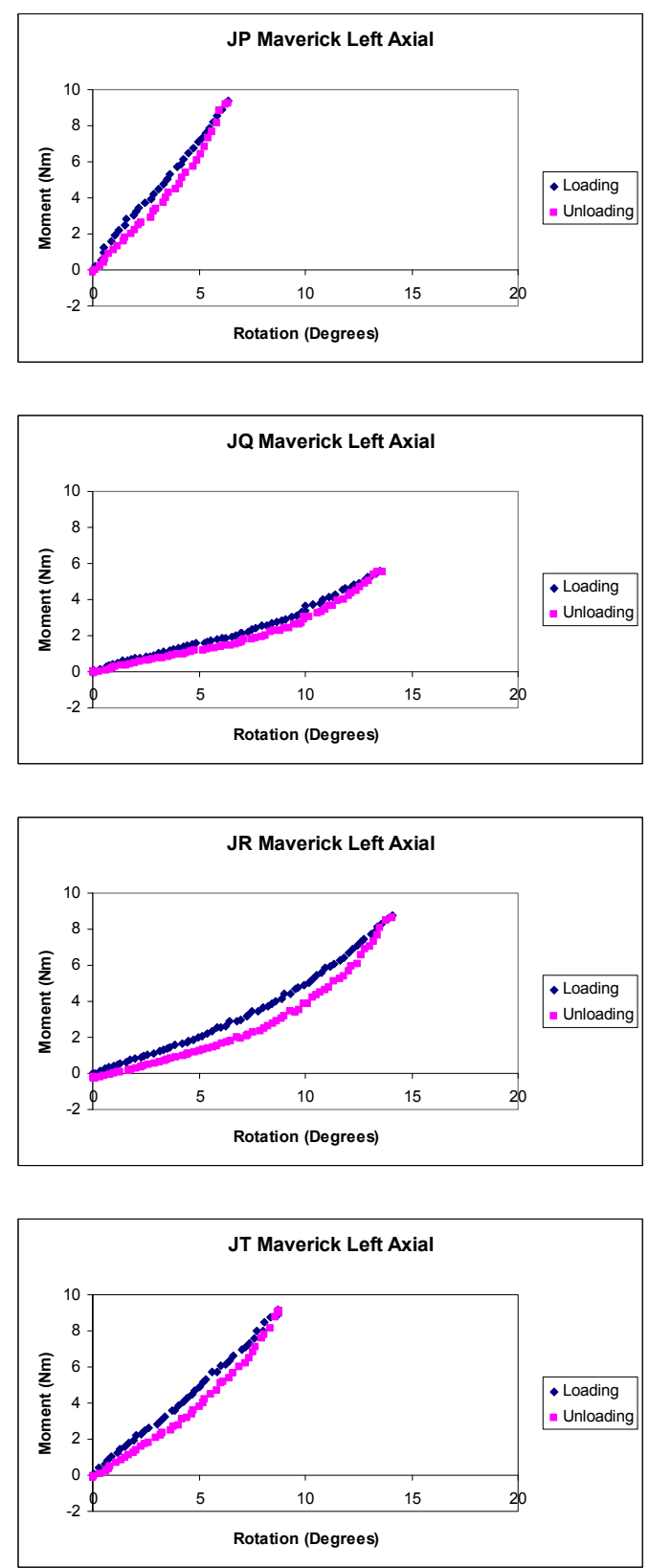
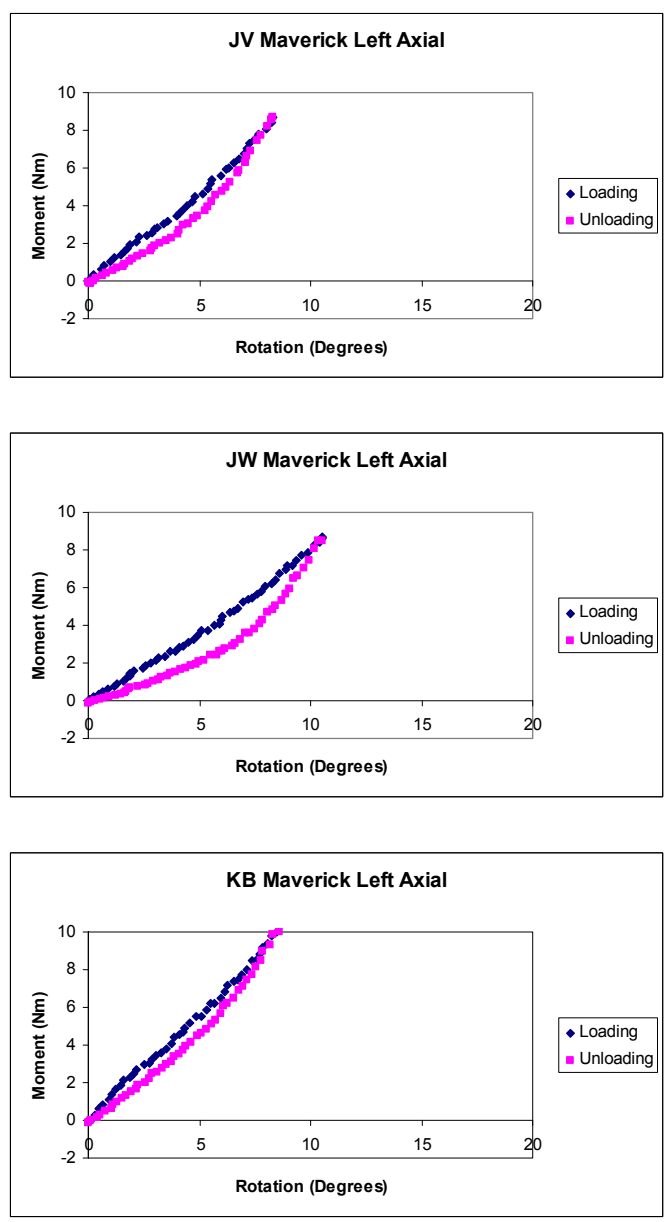

Figure D.19: Maverick Left Axial—Stiffness Curves. 

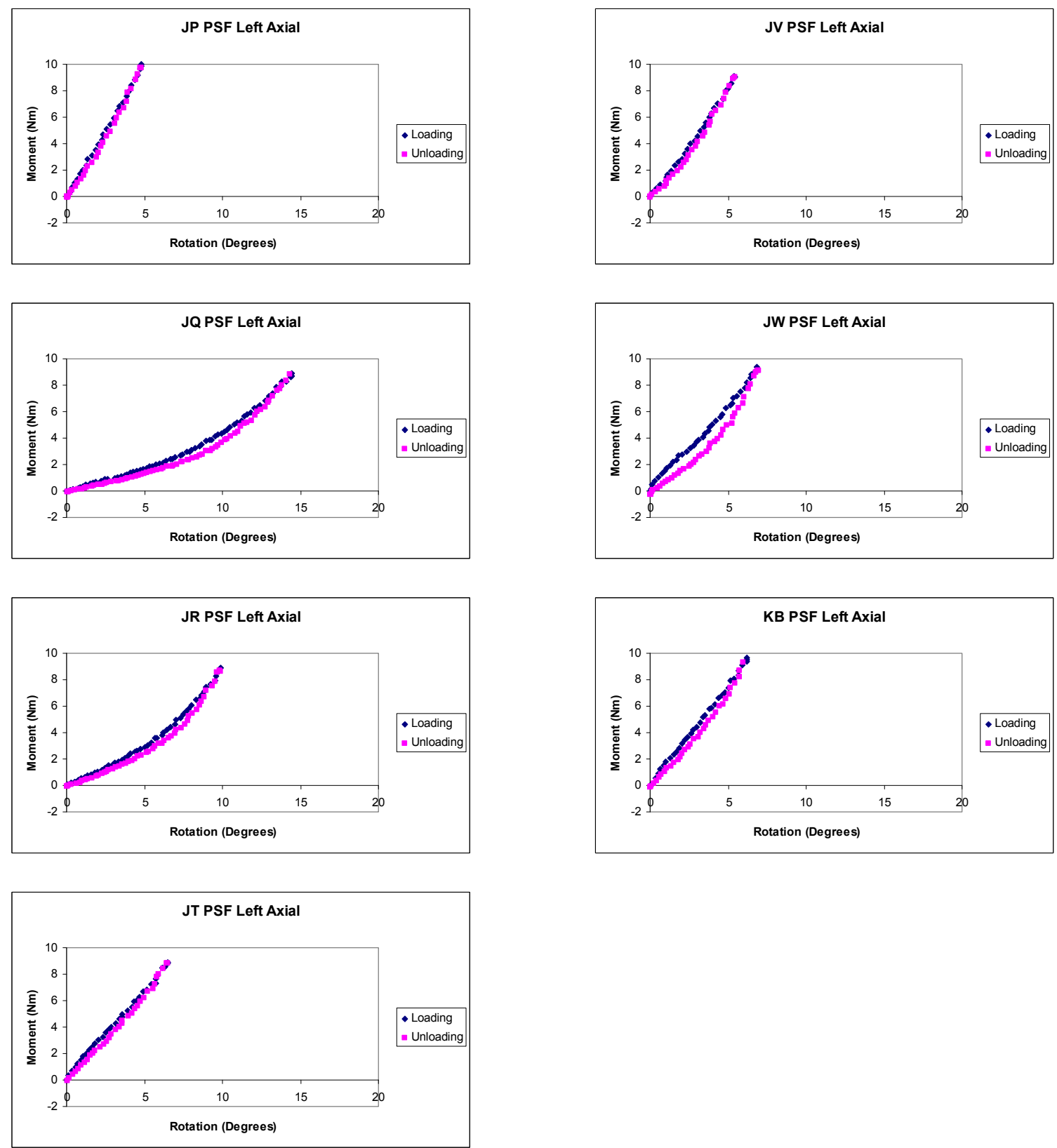

Figure D.20: Pedicle Screw FixationLeft Axial—Stiffness Curves. 

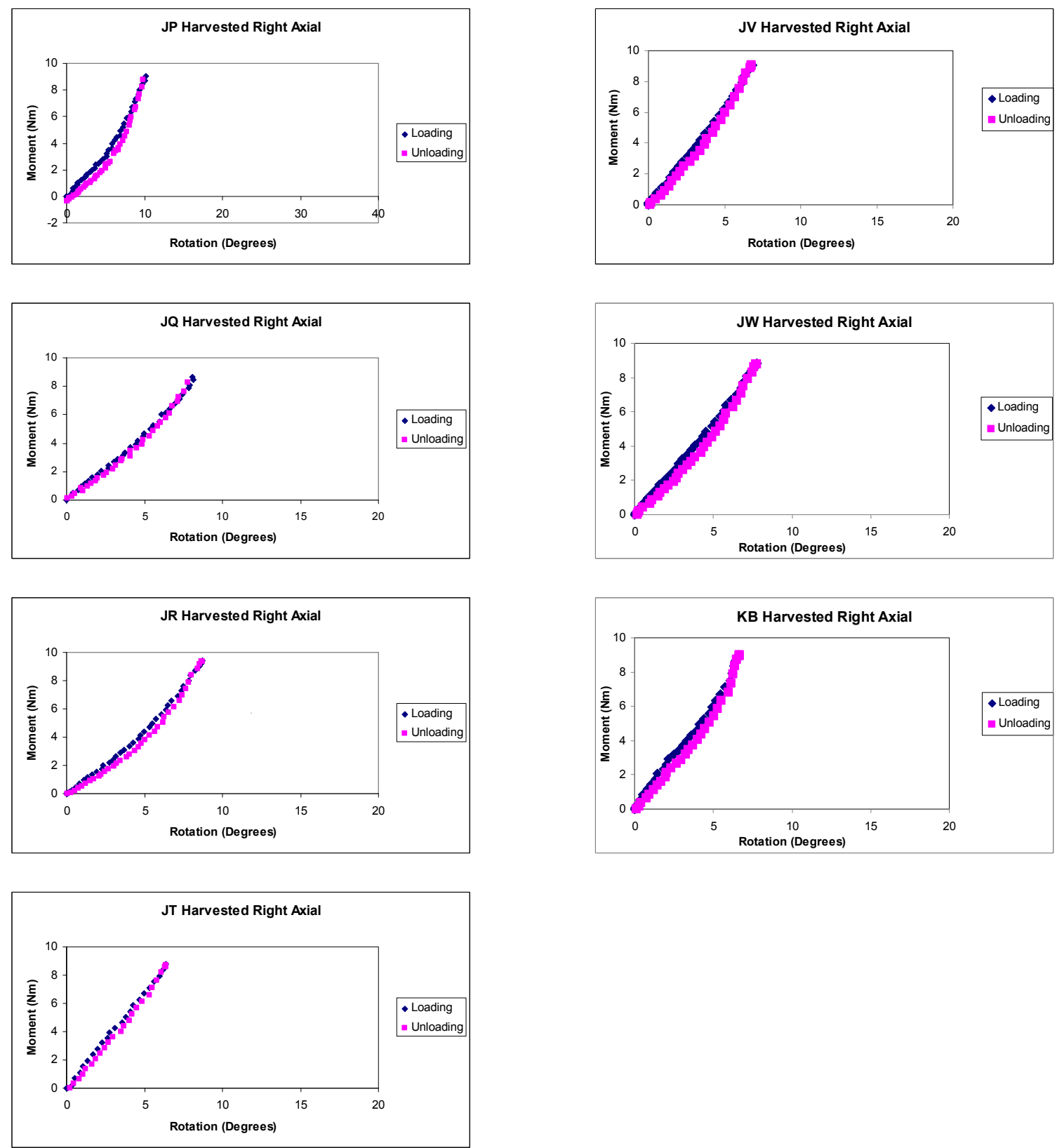

Figure D.21: Harvested Right Axial—Stiffness Curves. 

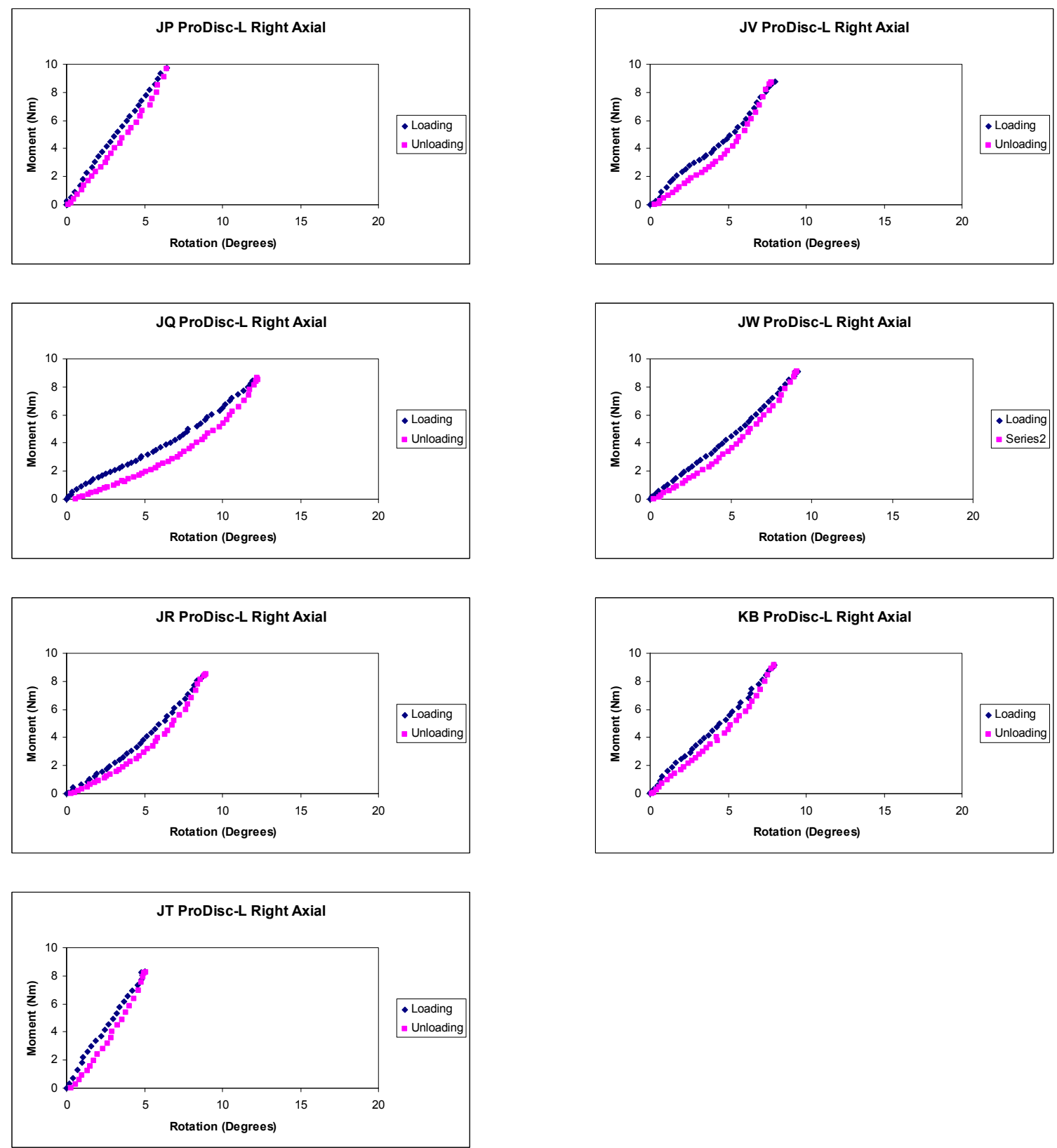

Figure D.22: ProDisc-L Right Axial—Stiffness Curves. 

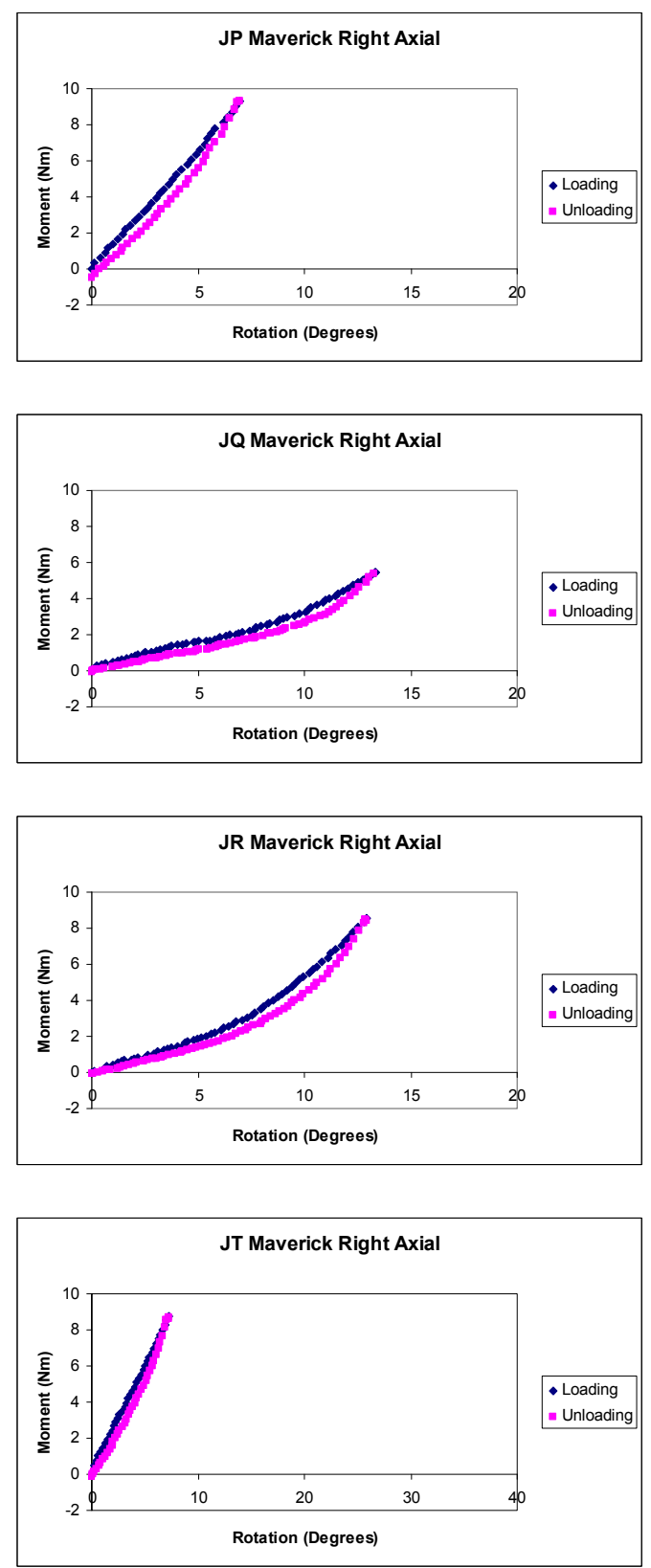
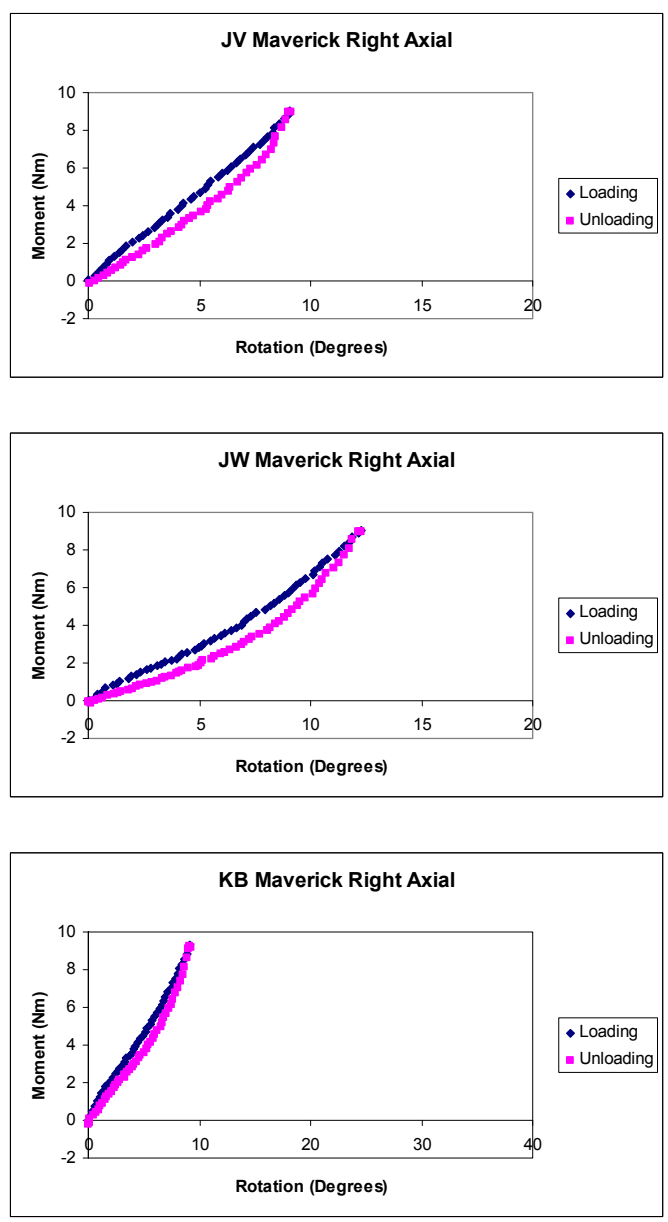

\section{Figure D.23: Maverick Right Axial—Stiffness Curves.}



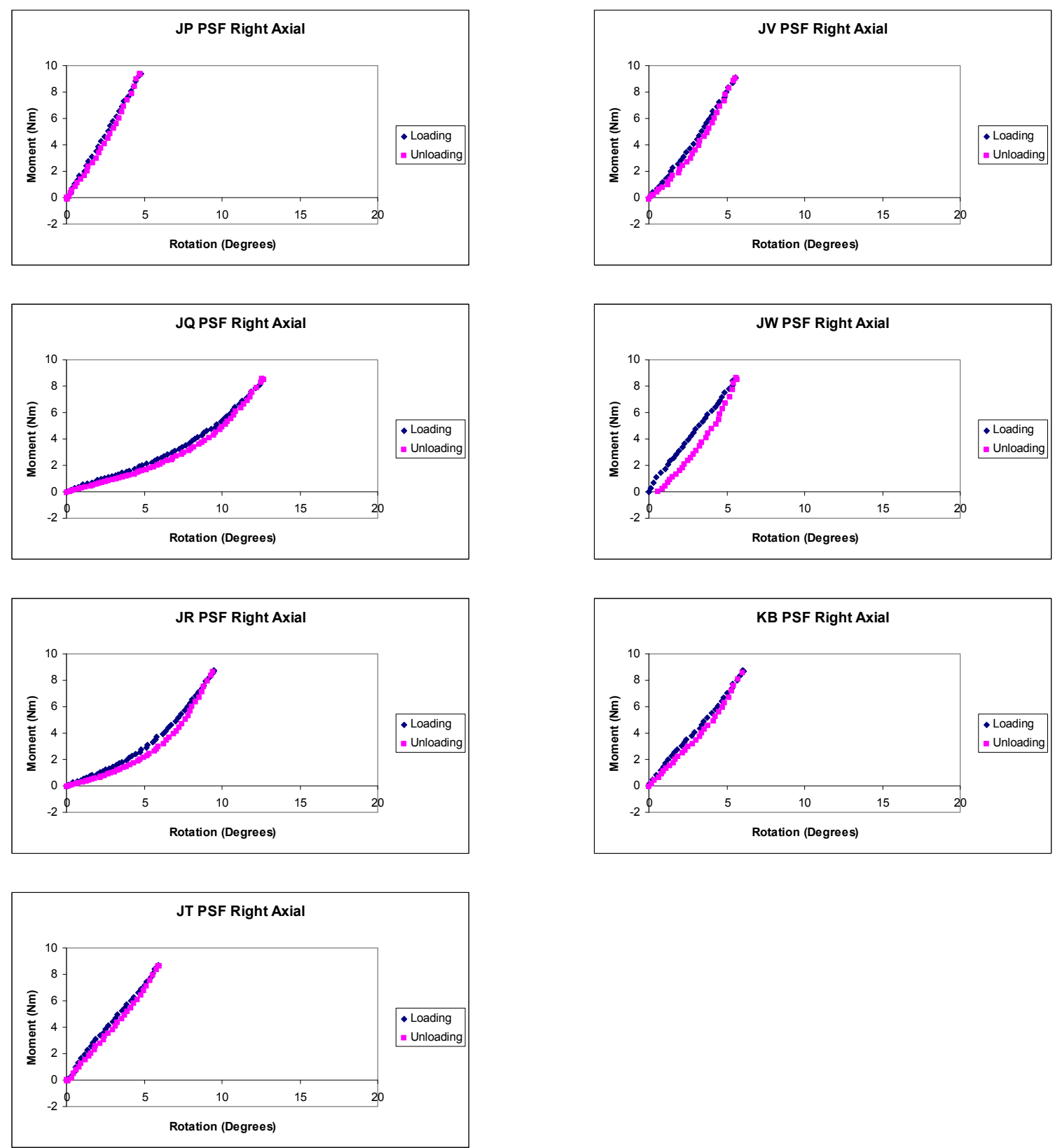

Figure D.24: Pedicle Screw Fixation Right Axial—Stiffness Curves. 


\section{VITA}

Peter Wong was born in Baltimore, Maryland on September 4, 1983. He moved to Memphis at a young age and spent most of his young life there. After graduating from White Station High School, he entered the University of Illinois, Urbana-Champaign in 2001. He received a Bachelor of Science degree in General Engineering in 2005 and soon afterwards enrolled in the joint biomedical engineering program at the University of Memphis and University of Tennessee Health Science Center. 